\title{
Medication optimisation
}

Citation for published version (APA):

de Wit, H. (2016). Medication optimisation: exploring the use of a clinical decision support system.

[Doctoral Thesis, Maastricht University]. Maastricht University. https://doi.org/10.26481/dis.20161130hw

Document status and date:

Published: 01/01/2016

DOI:

10.26481/dis.20161130hw

Document Version:

Publisher's PDF, also known as Version of record

\section{Please check the document version of this publication:}

- A submitted manuscript is the version of the article upon submission and before peer-review. There can be important differences between the submitted version and the official published version of record.

People interested in the research are advised to contact the author for the final version of the publication, or visit the DOI to the publisher's website.

- The final author version and the galley proof are versions of the publication after peer review.

- The final published version features the final layout of the paper including the volume, issue and page numbers.

Link to publication

\footnotetext{
General rights rights.

- You may freely distribute the URL identifying the publication in the public portal. please follow below link for the End User Agreement:

www.umlib.nl/taverne-license

Take down policy

If you believe that this document breaches copyright please contact us at:

repository@maastrichtuniversity.nl

providing details and we will investigate your claim.
}

Copyright and moral rights for the publications made accessible in the public portal are retained by the authors and/or other copyright owners and it is a condition of accessing publications that users recognise and abide by the legal requirements associated with these

- Users may download and print one copy of any publication from the public portal for the purpose of private study or research.

- You may not further distribute the material or use it for any profit-making activity or commercial gain

If the publication is distributed under the terms of Article $25 \mathrm{fa}$ of the Dutch Copyright Act, indicated by the "Taverne" license above, 

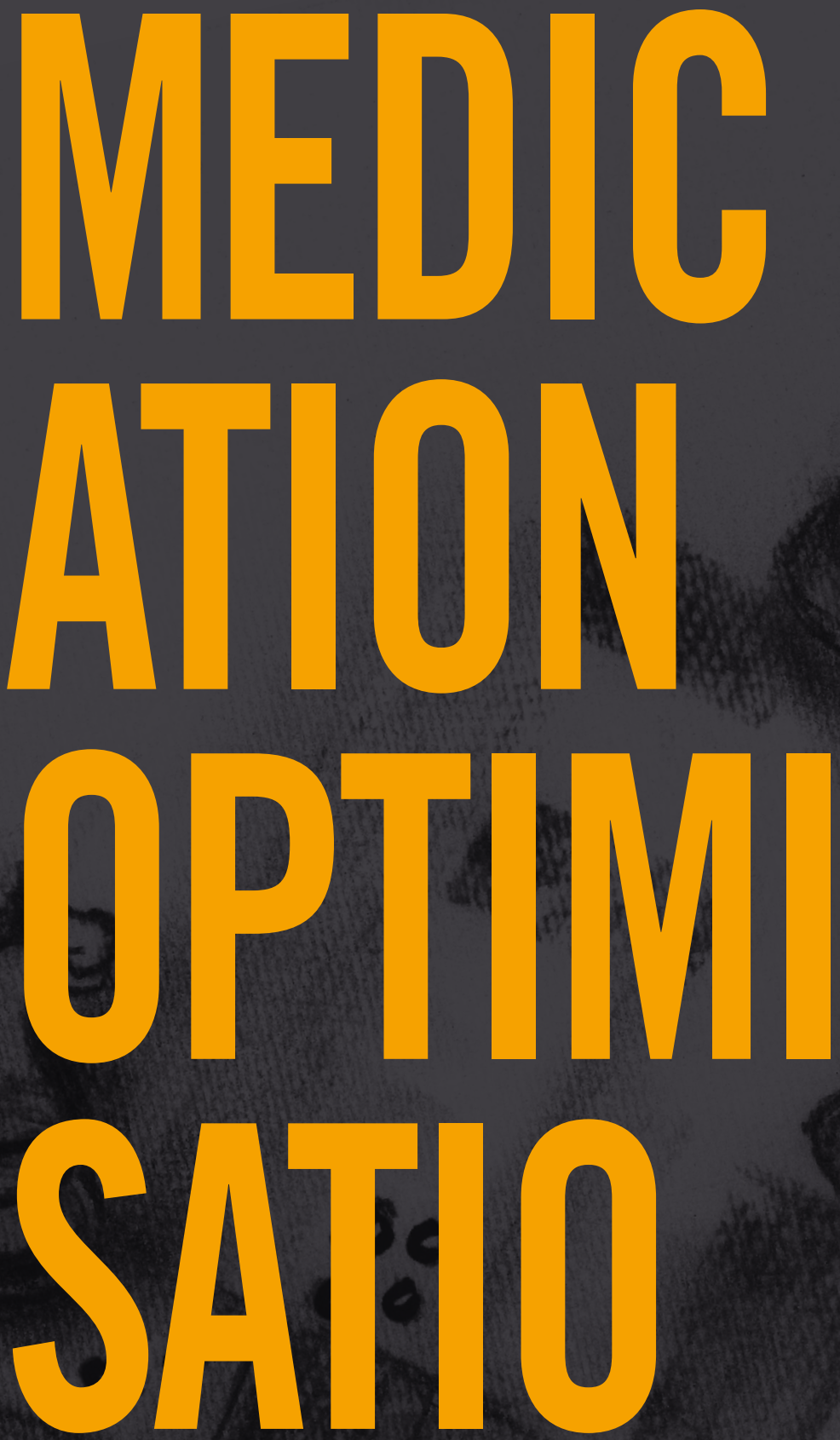

HUGO DE WIT

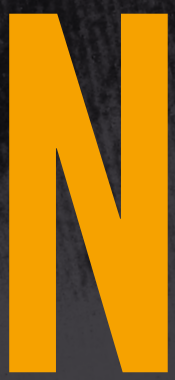

EXPLORING THE USE OF A CLINICAL DECISION SUPPORT SYSTEM 
ISBN 9789461596291 


\section{Medication Optimisation}

\section{Exploring the use of a clinical decision support system}

\section{PROEFSCHRIFT}

ter verkrijging van de graad van doctor aan de Universiteit Maastricht, op gezag van de Rector Magnificus, Prof dr. Rianne M. Letschert volgens het besluit van het College van Decanen,

in het openbaar te verdedigen op 30 november 2016 om 16 uur

door Hubertus Adrianus Johannes Maria de Wit 


\section{Promotor:}

Prof. dr. J.M.G.A. Schols

\section{Copromotores:}

dr. B. Winkens

dr. R. Janknegt, Zuyderland Medisch Centrum

dr. P-H.M. van der Kuy, Zuyderland Medisch Centrum

\section{Beoordelingscommissie:}

Prof. dr. J.F.M. Metsemakers (voorzitter)

Prof. dr. O. Bekers

Prof. dr. A.C.G. Egberts (Universiteit Utrecht)

Prof. dr. R.J. van Marum (Vrije Universiteit Amsterdam)

Prof. dr. C. Neef

This thesis was part of research project SCREEN which was supported by a grant of The Netherlands Organisation for Health Research and Development (ZonMW, grant number 113101001) 


\section{CONTENTS}

Chapter 1 Introduction and outline of the thesis

Chapter 2 Clinical practice of medication reviews in institutional care settings for older people in the Netherlands: an explorative survey

Chapter 3 Evaluation of clinical rules in a standalone pharmacy based clinical decision support system for hospitalised and nursing home patients

Chapter 4 Development of a computer system to support medication reviews in nursing homes

Chapter 5 The support of a medication review in hospitalised patients by a clinical decision support system

Chapter 6 The development of an automated ward independent delirium risk prediction model

Chapter 7 General Discussion

Summary

Samenvatting

Valorisation

Dankwoord

List of publications

Curriculum vitae 



\section{1}

Introduction and outline of the thesis 

Evidence-based medicine stresses the use of evidence from clinical research and emphasizes not to use intuition, pathophysiologic reasoning or personal clinical experience as a rationale for medical treatment (1). The first blinded randomized clinical trial, which forms the basis of current evidence-based medicine, was performed in 1946 for the treatment of pulmonary tuberculosis, and compared the use of both streptomycin and bed rest, to bed rest only (2). Although the term 'evidence-based medicine' was not used until the 1990s, the number of publications of clinical practice guidelines, including medication therapy, has increased published since the 1980s. The emergence of these clinical practice guidelines occurred parallel to an increase in the introduction of new drugs $(1,3,4)$. With a growing amount of available drugs, the simultaneous use of two drugs or more became quite common. Awareness of drug interactions arose after the occurrence of clinical problems. This was described for the first time in the 1960s in patients who were using an antidepressant called monoamine oxidase (MAO)-inhibitor and at the same time consumed cheese containing tyramine. This combination was reported to end in fatal hypertensive crises due to the inhibited metabolism of tyramine caused by the antidepressant, which allows tyramine to fully exert its effects on the blood pressure (5). The increased awareness of drug interactions has led to more publications on drug interactions and to the introduction of a classification system to differentiate between the clinically important interactions and the non-relevant interactions (5).

\section{CLINICAL DECISION SUPPORT SYSTEMS FOR MEDICATION SURVEILLANCE}

As with the increase in information and evidence-based data became a growing concern for healthcare professionals, a clinical decision support system (CDSS) provided a feasible solution to automatically alert against clinical problems such as drug-drug interactions. A CDSS is a computer program designed to support healthcare professionals in clinical decision-making. Alerts with patient specific recommendations are generated by matching individual patient characteristics to a knowledge-based system $(6,7)$. A CDSS incorporates a knowledge-based system which is a software engine that encodes concepts derived from experts, i.e. pre-defined clinical problems such as the prescription of two drugs that should not be used together, also known as a drug-drug interaction. A CDSS analyses the data and sends out alerts whenever necessary (8).

One of the first (electronic) clinical decision support systems implemented into actual healthcare situations that provided alerts of drug-drug interactions, was MEDIPHOR (Monitoring and Evaluation of Drug Interactions by a Pharmacy Oriented Reporting system). This system was introduced in 1973 in Stanford University Medical Centre and seemed to be a good educational tool with possibilities to reduce drug-interaction problems (9). In the Netherlands, the Royal Dutch Pharmacists Association (KNMP) initiated 
the development of a so-called 'medicines databank' in 1979, currently known as the Gstandard, which supports medication surveillance by alerting against incorrect dosages, drug-drug interactions, and contraindications (drug-disease interactions). Medication surveillance was defined as 'collecting information considering the patients total medication use, allowing the pharmacist to give specific advice to the patient and physician' (10). All pharmacists are currently required by the Dutch Healthcare Inspectorate (IGZ) to perform medication surveillance (11). Since 2014 Dutch physicians are also obliged by the IGZ to prescribe medication electronically by using a 'Computerized Physician Order Entry System' (CPOE) with an integrated drug safety alert system, which is described as an important element for successful implementation of a CDSS $(12,13)$. In the Netherlands, a CPOE integrated drug safety alert system is also provided by the KNMP-initiated medicines databank. Since the introduction of the first CDSS these systems have evolved from standalone decision support systems requiring pro-active accessing the CDSS and having to manual enter a single patient's characteristics, to a CDSS that is integrated in the primary hospital system by using an interface-oriented system without having to manually enter information (14). CDSSs can be categorised according to their architecture (14), however, also according to their 'clinical content'. The CDSS algorithms, also referred to as clinical rules, have been categorised according to four content types: process improvement, policy implementation, error prevention and decision support (15). With regard to medication surveillance, the medicines databank from the KNMP contains relatively simple algorithms that aim for error prevention and that is also considered to be the first generation of medication surveillance system. More advanced CDSSs also contain algorithms aiming for decision support, which is considered to be a characteristic for the second generation of medication surveillance systems $(16,17)$. Advanced CDSSs are also known to combine several sources of information on the characteristics of individual patients such as the renal function, while the KNMP medicines databank would only consider a patient with renal failure when this is specifically documented as a contraindication within the CPOE. However, these contraindications do not take the individual renal function into account since the laboratory values are not documented in the CPOE. Advanced CDSSs that take into account drug dosages in relation to the renal function have shown improvements in appropriate prescribing (18).

The efficiency of the CDSS, i.e. having a high percentage of clinically relevant alerts, is also an important element for the successful implementation of an advanced CDSS (12). A resultant of non-clinically relevant alerts from a CDSS is alert fatigue: ignoring of both relevant and not-relevant alerts. It has been reported that physicians ignore drug safety alerts in $49 \%$ to $96 \%$ of the cases (19), and also pharmacists are reported to ignore relevant alerts (20). The efficiency of alerts from the medicine databank has been reported to increase when augmented with an advanced CDSS $(17,21)$. However, the efficiency, i.e. pharmacists' interventions as a result of the alerts, of an advanced CDSS still remains low (16). The efficiency of advanced CDSSs should therefore be optimised 
before they are integrated into the prescribing workflow of a physician. The first step is determining how the efficiency of the advanced CDSSs, which are mostly home-grown (17), can be improved.

\section{POLYPHARMACY AND ELDERLY PEOPLE}

As people get older, the use of medication increases. In the Netherlands, $30-45 \%$ of the population over 65 years old uses more than 5 drugs and for $20 \%$ of the population over 75 years old this number increases to more than 9 drugs (22). The concomitant use of many drugs is also known as polypharmacy, which is often based on an arbitrary number of drugs used, but can also be defined as drugs used that are not medically indicated (23). Polypharmacy increases the risk of adverse effects, drug interactions, and often causes problems with patient compliance (24-27). Despite the use of a first generation CDSS for medication surveillance, polypharmacy is shown to be an independent risk factor for hospitalisation which can increase five- to nine-fold with the use of more than 3 drugs or 10 drugs, respectively $(28,29)$, and the use of 10 or more drugs is shown to be an independent risk factor for mortality (30). Moreover, polypharmacy increases the likelihood of inappropriate prescription and at the same time may cause suboptimal treatment due to the fact that the probability of under-prescription paradoxically increases with the number of drugs used $(31,32)$. Inappropriate medication was defined for the first time with the 'Beers criteria' developed by using a Delphi consensus with 13 recognized experts in a nursing home population (33). In communitydwelling older adults and nursing home residents, a high prevalence of $12 \%$ and $40 \%$ respectively, is found for potentially inappropriate medication (34). A resultant of inappropriate medication can be a drug-related problem (DRP) which is defined as "an event or a circumstance involving drug therapy that actually or potentially interferes with health care outcome" (35). Leendertse et al. have shown that 5.6\% of the unplanned hospital admissions in the Netherlands were medication related. It is likely that half of these hospital admissions could have been prevented and were thus a resultant of medication inappropriateness (28).

\section{MEDICATION REVIEWS}

A medication review, in this thesis referred to as a 'clinical medication review', can be described as the process in which a health professional reviews the patient's drug treatment in relation to his or her illness, whilst taking into account the therapeutic efficacy of each drug, adverse drug effects, interactions, compliance, and the patient's understanding of the illness and its treatment. The clinical medication review aims to 
optimise the patient's pharmacological treatment and eliminate medication inappropriateness, and thus aims to reduce preventable unplanned hospital admission $(28,36)$.

The Dutch Healthcare Inspectorate expects all residents of nursing homes and residential homes to receive a medication review by a physician and a pharmacist twice a year and yearly, respectively. In this medication review the information provided by the nursing staff and/or the patient him/herself should also be taken into account (37). It is likely that the often frail population of nursing homes and residential homes will benefit considerably from medication reviews.

A study by Zermansky et al. showed that an adequate medication review of residents in care homes may lead to a reduction in the number of falls. However, no significant change was found in other parameters such as mortality or the number of hospitalisations (38). A review by Alldred showed that regular revision of medication, including the performance of medication reviews, in residents of care homes reduced DRPs and improved medication appropriateness (39). When performing medication reviews among hospitalised patients, which is another clinical setting, also no conclusive benefits on readmission or decrease in mortality was shown. However, performing medication reviews did show a reduction in the number of emergency department contacts (40). A systematic review and meta-analysis published in 2007 concerning pharmacistled medication reviews of older people in any setting (mean age $>60$ years) showed no significant positive effects on mortality or hospital admissions. However, a slight decrease in the amount of prescribed drugs and an improvement of drug-knowledge and adherence were observed (41). Improved adherence can be very beneficial; e.g. in diabetes patients it has been shown that improved adherence to guidelines and drugadherence resulted in better prognosis and cost reduction $(42,43)$. Another study on the benefits of a medication review in a high risk elderly population in primary care (age $>80$ years with a minimum of 4 orally used medicines) also showed a reduction in the number of drugs prescribed (44). The reduction of prescribed drugs after a medication review can aid in more appropriate prescription and diagnosis $(45,46)$. The PHARMstudy (acronym for: 'Preventing Hospital Admissions by Reviewing Medication'), a controlled trial in the primary care setting, studied the effectiveness of a thorough medication review methodology based on a study by Hepler et al. (47), and showed that medication reviews may prevent medication related hospital admissions. This study was however underpowered to detect medication related hospital admissions (48). In a recent systematic review and meta-analysis by Hatah et al. of pharmacist led fee-for service medication reviews showed that specifically clinical medication reviews, which are the most comprehensive, resulted in reduced hospitalisation and positive impacts on LDL and blood pressure (49). The method used to perform medication reviews thereby appears to be very important. The importance of method, or tool, used to perform medication reviews is supported by the effects shown when using the geriatric palliative method (an algorithm for drug discontinuation in polypharmacy). This algorithm was effective in reducing polypharmacy as well as mortality and morbidity in nurs- 
ing home patients (50). Other tools have shown their benefits by improving appropriate prescribing in polypharmacy patients. A tool such as the POM (acronym for 'Prescribing Optimisation Method') consists of six open questions that support the physician in checking whether patients receive the best pharmacologic treatment possible. The POM tool has shown to increase the proportion of correct medication related decisions by general practitioners from $34.7 \%$ to $48.1 \%$ in geriatric polypharmacy patients (51). The STOPP/START (acronyms for 'Screening Tool of Older Persons' Potentially inappropriate prescriptions' and 'Screening Tool to Alert to Right Treatment', respectively) criteria are based on a Delphi consensus method and on the evidence-based prescribing indicator. In hospitalised patients aged > 65 years, the STOPP/START criteria have had a positive effect on the 'medication appropriateness index' (MAI) and 'assessment of underutilisation index' (AOU) with an absolute risk reduction of $35.7 \%$ and $21.2 \%$, respectively (52). Recently this tool also has shown to reduce adverse drug reactions and length of hospitalisation (53). In a recent systematic review the above described tools have demonstrated a positive effect with regard to drug therapy optimisation and can thereby provide basic support for medication reviews. However, these methods used appear to lack supremacy to show convincing clinical effects in studies, such as reduced hospitalisation. Moreover, most studies included were described to be of poor quality and small sample sizes (54). It seems reasonable to assume that a reviewing tool is only as good as the health care professional using it. Outcomes will depend on variables such as knowledge of evidence-based guidelines or perhaps the focus of the healthcare professional. Moreover, the methods to support medication reviews appear to be timeconsuming and have temporary effects since they are mostly used manually and infrequently. A solution for the practical problems arising from the increasing amount of evidence-based data, the growing number of older adults that use more drugs, and the obligation to perform medication reviews in extremely large groups of patients might be a CDSS. A CDSS would be able to perform its surveillance around the clock, regardless whether it is for medication surveillance or medication reviews, without any limitations on the number of patients, and it would be consistent into applying evidencebased data, i.e. finding the pre-defined medication inappropriateness.

\section{CLINICAL DECISION SUPPORT SYSTEMS IN PREDICTIVE MEDICINE}

Aside from medication surveillance implemented into advanced CDSSs, clinical content can differ widely, for example: aiding the choice of therapy for paediatric pulmonologists, interventions aiding in therapy of pressure ulcers and malnutrition, or prevention of veneus trombo-embolic events by alerting for at-risk patients (55-57). Studies have also shown that clinical practice guidelines integrated in CDSSs, help healthcare professionals to avoid errors and improve clinical practice and quality of care $(55,58,59)$. These CDSSs contain clinical practice guidelines that rely on similar aspects 
of the currently manually performed medication reviews. It might be possible that a CDSS that contains algorithms to detect medication inappropriatenesses known from medication reviews would be able to support medication reviews.

In addition to the implementation of clinical practice guidelines, a CDSS can also be used on an individual level for subacute disease risk prediction models. If an individual's risk for a specific disease can be predicted then personalised treatment strategies can be initiated (60). Stusser et al. proposed that the automated presentation of the realtime health status of a patient in terms of disease risk prediction could support the physician. Automated presentation can be done by using patient data from clinical systems (61). An example of the use of a CDSS for risk prediction for diseases is the alerting of neonatal sepsis before clinical signs of illness are recognized. This is done by combining the laboratory values and the electrocardiogram of the neonate for changes in the heart rate characteristics. This method has already demonstrated to be able to reduce mortality by $20 \%$ in low birth weight neonates (62). It is suggested that also other predictive algorithms for subacute complications or illnesses such as relapse of congestive heart failure could be developed (63).

The development of an automated predictive algorithm aiming for an illness with a clear relevancy for clinical practice might be considered. Delirium is a disorder of cognition and attention which may be observed in older adults after hospitalisation and is a sign of the brain's vulnerability to external factors (64). The incidence of delirium in hospitalised older adults is high (3-29\%) and is associated with high costs ranging from $\$ 16303$ to $\$ 64421$ per patient. Furthermore, delirium is associated with increased morbidity and mortality (64-66). Non-pharmacologic treatment has been shown to be effective in reducing delirium incidence in hospitalised older patients, while evidence for prevention or treatment with antipsychotics remains unclear (67-69). Delirium is known to be multifactorial in older adults and many risk factors have been included in delirium risk prediction models in order to start timely treatment (70). However, at the moment these risk prediction models are calculated manually (71-74). As mentioned earlier, in the beginning CDSSs were implemented as standalone systems that required pro-active seeking of the system while also needing to manual enter a single patient's information. Having to pro-actively calculate an individual's risk for delirium is a much larger barrier compared to having the CDSS automatically present the individual's risk for delirium. Likewise, initiating preventive treatment also requires the health care professional to consciously determine whether a patient is at risk. In other words, if he or she does not realise that the patient is at risk, the physician will neglect to use nonautomated predictive models (14). Currently the commonly used predictive variables used in delirium risk prediction models, such as age, cognitive disorders, illness severity, vision impairment, infections, dehydration, and certain drugs (70-72,75-77), are not altogether available electronically. However, despite this issue measures to improve detection of delirium are required, since many delirium diagnosis are missed in clinical practice (66). Thus, the development of an automated risk prediction model which 
includes the current electronically available predictive variables, appears useful considering the multifactorial aspect of delirium, the availability of effective preventive (nonpharmacologic) treatment, and the importance of the disease which is reflected by high incidence, increased morbidity and mortality.

\section{OBJECTIVES OF THIS THESIS}

The objectives of this thesis are fourfold. The first objective is to identify the current efforts as well as the extra efforts required to perform the compulsory medication reviews, in order to in order to properly identify the need for automated support of the complex process of medication reviews.

The second objective is to determine which aspects are the most important for improving the efficiency of an advanced CDSS used in medication surveillance.

The third objective of this thesis is to explore the possibilities of using an advanced CDSS to support medication reviews. The fourth objective is to explore the possibilities of using a CDSS for the prediction of an important subacute illness in hospitalised patients, i.e. the development of a delirium predictive algorithm in hospitalised older adults.

\section{OUTLINE OF THIS THESIS}

Following the general introduction, Chapter 2 describes an explorative survey among pharmacists related to the clinical practice of medication reviews for older people living in residential and nursing homes. The survey takes into account to what extent medication reviews are performed as required by the Dutch Healthcare Inspectorate, the time needed to perform the required medication reviews, and the opinion of the pharmacist on whether medication reviews are economically efficient and medically relevant. These questions focus on revealing the present state of performing medication reviews in the Netherlands, thereby assessing the need for support of medication reviews by a CDSS.

Chapter 3 describes the identification and quantification of the positive and negative properties of a home-grown advanced CDSS with algorithms that aim to improve medication surveillance. The quantification of the positive and negative properties will show the efficiency of the CDSS and ways to improve it. Such improvements are necessary to further develop the efficiency of medication surveillance while using an advanced CDSS. Moreover, an efficient advanced CDSS is a pre-requisite for its use in the support of medication reviews.

The development of a CDSS to support medication reviews and the use of a CDSS for further optimisation and improvement of drug therapy is described in more detail in Chapter 4. 


\section{Chapter 1}

The benefits of a CDSS developed to support medication reviews in comparison to manually performed medication reviews are studied in Chapter 5. This comparison explores the value of the CDSS in the performance of medication reviews in hospitalized older adults, and also identifies limitations found in the CDSS that need to be overcome to optimally support medication reviews by using a CDSS

Chapter 6 explores the possibility of using a CDSS for predictive medicine in a subacute illness. This chapter describes the development of an automated delirium prediction model that enables the individual risk prediction of a delirium in a hospital setting. Furthermore, this chapter shows the limitations of the currently available patient characteristics stored for automation in hospital systems.

Finally, Chapter 7, the general discussion, describes the results of the above mentioned studies and puts them in a broader perspective in relation to future automation of medication surveillance, medication reviews, clinical practice and patient outcomes. 


\section{REFERENCES}

1. Evidence-Based Medicine Working Group. Evidence-based medicine. A new approach to teaching the practice of medicine. JAMA. 1992;268(17):2420-5.

2. Bhatt A. Evolution of Clinical Research: A History Before and Beyond James Lind. Perspec.Clin. Res. 2010;1(1):6-10.

3. Weisz G, Cambrosio A, Keating P, Knaapen L, Schlich T, Tournay VJ. The emergence of clinical practice guidelines. The Milbank Q. 2007;85(4):691-727.

4. Kinch MS, Haynesworth A, Kinch SL, Hoyer D. An overview of FDA-approved new molecular entities: 1827-2013. Drug Discov Today. 2014;19(8):1033-9.

5. Sjöqvist F, Böttiger Y. Historical perspectives: drug interactions - it all began with cheese. J Intern Med. 2010;268(6):512-5.

6. Sim I, Gorman P, Greenes RA, Haynes RB, Kaplan B, Lehmann H et al. Clinical decision support systems for the practice of evidence-based medicine. J Am Med Inform Assoc. 2001;8(6):527-34.

7. Shortliffe EH. Computer programs to support clinical decision making. JAMA. 1987;258(1):61-6.

8. Musen MA, Shahar Y, Shortliffe EH. Clinical Decision-Support Systems. In: Shortliffe EH, Cimino JJ, editors. Biomedical Informatics: Computer Applications in Health Care and Biomedicine. New York, NY: Springer New York; 2006. p. 698-736.

9. Morrell J, Podlone M, Cohen SN. Receptivity of physicians in a teaching hospital to computerized drug interaction monitoring and reporting system. Med Care. 1977;15(1):68-78.

10. Winters DJ. KNMP-bestand: nieuwe basis voor Informatorium and Taxen. Pharm Weekbl. 1979(114):353-8.

11. KNMP. Handboek medicatiebewaking [Internet]. 2013. [cited on 20 Nov. 2015] Available from: https://www.knmp.nl/patientenzorg/medicatiebewaking/handboek-medicatiebewaking-2013.

12. Scheepers-Hoeks AM, Grouls RJ, Neef C, Korsten HH. Strategy for implementation and first results of advanced clinical decision support in hospital pharmacy practice. Stud Health Technol Inform. 2009;148:142-8.

13. KNMG. Richtlijn elektronisch voorschrijven [Internet]. 2013. [cited on 20 Nov 2015] Available from: http://knmg.artsennet.nl/Publicaties/KNMGpublicatie/136411/Richtlijn-elektronisch-voorschrijven2013.htm.

14. Wright A, Sittig DF. A four-phase model of the evolution of clinical decision support architectures. Int J Med Inform. 2008;77(10):641-9.

15. Berlin A, Sorani M, Sim I. A taxonomic description of computer-based clinical decision support systems. J Biomed Infor. 2006;39(6):656-67.

16. Rommers MK, Teepe-Twiss IM, Guchelaar HJ. A computerized adverse drug event alerting system using clinical rules: a retrospective and prospective comparison with conventional medication surveillance in the Netherlands. Drug Saf. 2011;34(3):233-42.

17. Helmons PJ, Suijkerbuijk BO, Nannan Panday PV, Kosterink JGW. Doing the right things and doing things right: inpatient drug surveillance assisted by clinical decision support. Eur J Hosp Pharm. 2015;22(4):236-42.

18. Tawadrous D, Shariff SZ, Haynes RB, lansavichus AV, Jain AK, Garg AX. Use of clinical decision support systems for kidney-related drug prescribing: a systematic review. Am J Kidney Dis. 2011;58(6):903-14.

19. van der Sijs H, Aarts J, Vulto A, Berg M. Overriding of drug safety alerts in computerized physician order entry. J Am Med Inform Assoc. 2006;13(2):138-47.

20. Bates DW, Kuperman GJ, Wang S, Gandhi T, Kittler A, Volk L et al. Ten Commandments for Effective Clinical Decision Support: Making the Practice of Evidence-based Medicine a Reality. J Am Med Inform Assoc. 2003;10(6):523-30.

21. Eppenga WL, Derijks HJ, Conemans JM, Hermens WA, Wensing M, De Smet PA. Comparison of a basic and an advanced pharmacotherapy-related clinical decision support system in a hospital care setting in the Netherlands. J Am Med Inform Assoc. 2012;19(1):66-71.

22. Lemmens LC, Weda M. Polypharmacie bij kwestbare ouderen: inventarisatie van risico's en mogelijke interventiestrategieen [Internet]. 2013 RIVM. [ cited on 20 nov 2015] Available from: http://www.rivm.nl/ 


\section{Chapter 1}

Documenten_en_publicaties/Wetenschappelijk/Rapporten/2013/juli/Polyfarmacie_bij_kwetsbare_ouder en_Inventarisatie_van_risico_s_en_mogelijke_interventiestrategie\%C3\%ABn.

23. Maher RL, Hanlon J, Hajjar ER. Clinical consequences of polypharmacy in elderly. Expert Opin Drug Saf. 2014;13(1):57-65.

24. Gandhi TK, Weingart SN, Borus J, Seger AC, Peterson J, Burdick E et al. Adverse drug events in ambulatory care. N Engl J Med. 2003;348(16):1556-64.

25. Tsai KT, Chen JH, Wen CJ, Kuo HK, Lu IS, Chiu LS et al. Medication adherence among geriatric outpatients prescribed multiple medications. Am J Geriatr Pharmacother. 2012;10(1):61-8.

26. Field TS, Gurwitz JH, Avorn J, McCormick D, Jain S, Eckler M et al. Risk factors for adverse drug events among nursing home residents. Arch Intern Med. 2001;161(13):1629-34.

27. Nobili A, Pasina L, Tettamanti M, Lucca U, Riva E, Marzona I et al. Potentially severe drug interactions in elderly outpatients: results of an observational study of an administrative prescription database. J Clin Pharm Ther. 2009;34(4):377-86.

28. Leendertse AJ, Egberts AC, Stoker LJ, van den Bemt PM, Group HS. Frequency of and risk factors for preventable medication-related hospital admissions in the Netherlands. Arch Intern Med. 2008;168(17): 1890-6.

29. Pedrós C, Quintana B, Rebolledo M, Porta N, Vallano A, Arnau J. Prevalence, risk factors and main features of adverse drug reactions leading to hospital admission. Eur J Clin Pharmacol. 2014;70(3):361-7.

30. Herr M, Robine JM, Pinot J, Arvieu JJ, Ankri J. Polypharmacy and frailty: prevalence, relationship, and impact on mortality in a French sample of 2350 old people. Pharmacoepidemiol Drug Saf. 2015;24(6):637-46.

31. Gallagher PF, Barry PJ, Ryan C, Hartigan I, O'Mahony D. Inappropriate prescribing in an acutely ill population of elderly patients as determined by Beers' Criteria. Age Ageing. 2008;37(1):96-101.

32. Cherubini A, Corsonello A, Lattanzio F. Underprescription of Beneficial Medicines in Older People: Causes, Consequences and Prevention. Drugs Aging. 2012;29(6):463-75.

33. Beers MH, Ouslander JG, Rollingher I, Reuben DB, Brooks J, Beck JC. Explicit criteria for determining inappropriate medication use in nursing home residents. UCLA Division of Geriatric Medicine. Arch Intern Med. 1991;151(9):1825-32.

34. Gallagher P, Barry P, O'Mahony D. Inappropriate prescribing in the elderly. J Clin Pharm Ther. 2007;32(2):113-21.

35. Silva C, Ramalho C, Luz I, Monteiro J, Fresco P. Drug-related problems in institutionalized, polymedicated elderly patients: opportunities for pharmacist intervention. Int J Clin Pharm. 2015;37(2):327-34.

36. Zermansky A, Petty D, Raynor T, Lowe C, Freemantle N. Clinical medication review by a pharmacist of patients on repeat prescriptions in general practice: a randomised controlled trial. Health Technol Assess. 2002;6(20):86.

37. Dutch Healthcare Inspectorate. Beoordelingskader medicatieveiligheid verpleeghuizen [Internet]. [cited on 01-09-2011]. Available from: http://www.igz.nl/Images/Bijlagen\%20Beoordelingskaders\%20en\%20 Risicoscore\%20bij\%20rapport\%20Medicatieveiligheid_tcm294-287277.pdf.

38. Zermansky AG, Alldred DP, Petty DR, Raynor DK, Freemantle N, Eastaugh J et al. Clinical medication review by a pharmacist of elderly people living in care homes--randomised controlled trial. Age Ageing. 2006;35(6):586-91.

39. Alldred DP, Raynor DK, Hughes C, Barber N, Chen TF, Spoor P. Interventions to optimise prescribing for older people in care homes. Cochrane Database Syst Rev. 2013;2:CD009095.

40. Christensen $M$, Lundh A. Medication review in hospitalised patients to reduce morbidity and mortality. Cochrane Database Syst Rev. 2013;2:CD008986.

41. Holland R, Desborough J, Goodyer L, Hall S, Wright D, Loke YK. Does pharmacist-led medication review help to reduce hospital admissions and deaths in older people? A systematic review and meta-analysis. $\mathrm{Br}$ J Clin Pharmacol. 2008;65(3):303-16.

42. Encinosa WE, Bernard D, Dor A. Does prescription drug adherence reduce hospitalizations and costs? The case of diabetes. Adv Health Econ Health Serv Res. 2010;22:151-73. 
43. Oh SW, Lee HJ, Chin HJ, Hwang JI. Adherence to clinical practice guidelines and outcomes in diabetic patients. Int J Qual Health Care. 2011;23(4):413-9.

44. Lenaghan E, Holland R, Brooks A. Home-based medication review in a high risk elderly population in primary care--the POLYMED randomised controlled trial. Age Ageing. 2007;36(3):292-7.

45. Tinetti ME, Bogardus ST, Agostini JV. Potential Pitfalls of Disease-Specific Guidelines for Patients with Multiple Conditions. New Engl J Med. 2004;351(27):2870-4.

46. Gijsen R, Hoeymans N, Schellevis FG, Ruwaard D, Satariano WA, van den Bos GAM. Causes and consequences of comorbidity: A review. J Clin Epidemiol. 2001;54(7):661-74.

47. Hepler CD, Strand LM. Opportunities and responsibilities in pharmaceutical care. Am J Hosp Pharm. 1990;47(3):533-43.

48. Leendertse AJ, de Koning GHP, Goudswaard AN, Belitser SV, Verhoef M, de Gier HJ et al. Preventing hospital admissions by reviewing medication (PHARM) in primary care: an open controlled study in an elderly population. J Clin Pharm Therap. 2013;38(5):379-87.

49. Hatah E, Braund R, Tordoff J, Duffull SB. A systematic review and meta-analysis of pharmacist-led fee-forservices medication review. Br J Clin Pharmacol. 2014;77(1):102-15.

50. Garfinkel D, Mangin D. Feasibility Study of a Systematic Approach for Discontinuation of Multiple Medications in Older Adults: Addressing Polypharmacy. Arch Intern Med. 2010;170(18):1648-54.

51. Drenth-van Maanen AC, van Marum RJ, Knol W, van der Linden CM, Jansen PA. Prescribing optimization method for improving prescribing in elderly patients receiving polypharmacy: results of application to case histories by general practitioners. Drugs Aging. 2009;26(8):687-701.

52. Gallagher PF, O'Connor MN, O'Mahony D. Prevention of Potentially Inappropriate Prescribing for Elderly Patients: A Randomized Controlled Trial Using STOPP/START Criteria. Clin Pharmacol Ther. 2011;89(6):845-54.

53. O'Mahony D, O'Sullivan D, Byrne S, O'Connor MN, Ryan C, Gallagher P. STOPP/START criteria for potentially inappropriate prescribing in older people: version 2. Age Ageing. 2015;44(2):213-8.

54. Cooper JA, Cadogan CA, Patterson SM, Kerse N, Bradley MC, Ryan C et al. Interventions to improve the appropriate use of polypharmacy in older people: a Cochrane systematic review. BMJ open. 2015;5(12):e009235.

55. Fossum M, Alexander GL, Ehnfors M, Ehrenberg A. Effects of a computerized decision support system on pressure ulcers and malnutrition in nursing homes for the elderly. Int J Med Inform. 2011;80(9):607-17.

56. Lomotan EA, Hoeksema LJ, Edmonds DE, Ramirez-Garnica G, Shiffman RN, Horwitz LI. Evaluating the use of a computerized clinical decision support system for asthma by pediatric pulmonologists. Int J Med Inform. 2012;81(3):157-65.

57. Kucher N, Koo S, Quiroz R, Cooper JM, Paterno MD, Soukonnikov B et al. Electronic alerts to prevent venous thromboembolism among hospitalized patients. N Engl J Med. 2005;352(10):969-77.

58. Jaspers MW, Smeulers M, Vermeulen H, Peute LW. Effects of clinical decision-support systems on practitioner performance and patient outcomes: a synthesis of high-quality systematic review findings. J Am Med Inform Assoc. 2011;18(3):327-34.

59. Raschke RA, Gollihare B, Wunderlich TA, Guidry JR, Leibowitz Al, Peirce JC et al. A computer alert system to prevent injury from adverse drug events: development and evaluation in a community teaching hospital. JAMA. 1998;280(15):1317-20.

60. Oh SM, Stefani KM, Kim HC. Development and application of chronic disease risk prediction models. Yonsei Med J. 2014;55(4):853-60.

61. Stusser R, Dickey R. Quality and Cost Improvement of Healthcare via Complementary Measurement and Diagnosis of Patient General Health Outcome Using Electronic Health Record Data: Research Rationale and Design. J Med Syst. 2013;37(6):1-13.

62. Fairchild KD. Predictive monitoring for early detection of sepsis in neonatal ICU patients. Curr Opin Pediatr. 2013;25(2):172-9.

63. Xiao Y, Griffin MP, Lake DE, Moorman JR. Nearest neighbor and logistic regression analyses of clinical and heart rate characteristics in the early diagnosis of neonatal sepsis. Medical decision making. Med Decis Making. 2010;30(2):258-66. 


\section{Chapter 1}

64. Inouye SK, Westendorp RG, Saczynski JS. Delirium in elderly people. Lancet. 2014;383(9920):911-22.

65. Leslie DL, Marcantonio ER, Zhang Y, Leo-Summers L, Inouye SK. One-year health care costs associated with delirium in the elderly population. Arch Intern Med. 2008;168(1):27-32.

66. Siddiqi N, House AO, Holmes JD. Occurrence and outcome of delirium in medical in-patients: a systematic literature review. Age Ageing. 2006;35(4):350-64.

67. Hshieh TT, Yue J, Oh E, Puelle M, Dowal S, Travison T et al. Effectiveness of multicomponent nonpharmacological delirium interventions: a meta-analysis. JAMA internal medicine. 2015;175(4):512-20.

68. Neufeld KJ, Yue J, Robinson TN, Inouye SK, Needham DM. Antipsychotic Medication for Prevention and Treatment of Delirium in Hospitalized Adults: A Systematic Review and Meta-Analysis. J Am Geriatr Soc. 2016;64(4):705-14.

69. Schrijver EJ, de Graaf K, de Vries OJ, Maier AB, Nanayakkara PW. Efficacy and safety of haloperidol for inhospital delirium prevention and treatment: A systematic review of current evidence. Eur J Intern Med. 2016;27:14-23.

70. Inouye SK. Predisposing and precipitating factors for delirium in hospitalized older patients. Dement Geriatr Cogn Disord. 1999;10(5):393-400.

71. Inouye SK, Viscoli CM, Horwitz RI, Hurst LD, Tinetti ME. A predictive model for delirium in hospitalized elderly medical patients based on admission characteristics. Ann Intern Med. 1993;119(6):474-81.

72. Carrasco MP, Villarroel L, Andrade M, Calderon J, Gonzalez M. Development and validation of a delirium predictive score in older people. Age Ageing. 2014;43(3):346-51.

73. van den Boogaard M, Pickkers P, Slooter AJ, Kuiper MA, Spronk PE, van der Voort PH et al. Development and validation of PRE-DELIRIC (PREdiction of DELIRium in ICu patients) delirium prediction model for intensive care patients: observational multicentre study. BMJ. 2012;344:e420.

74. Douglas VC, Hessler CS, Dhaliwal G, Betjemann JP, Fukuda KA, Alameddine LR et al. The AWOL tool: derivation and validation of a delirium prediction rule. J Hosp Med. 2013;8(9):493-9.

75. Laurila JV, Laakkonen ML, Tilvis RS, Pitkala KH. Predisposing and precipitating factors for delirium in a frail geriatric population. J Psychosom Res. 2008;65(3):249-54.

76. Young J, Leentjens AF, George J, Olofsson B, Gustafson Y. Systematic approaches to the prevention and management of patients with delirium. J Psychosom Res. 2008;65(3):267-72.

77. Young J, Murthy L, Westby M, Akunne A, O'Mahony R. Diagnosis, prevention, and management of delirium: summary of NICE guidance. BMJ. 2010;341. 


\section{Chapter}

\section{Clinical practice of medication reviews in institutional care settings for older people in the Netherlands: an explorative survey}

This chapter is based on:

de Wit HAJM, Winkens B, Mestres Gonzalvo C, Hurkens KPGM, Janknegt R, Schols JMGA, van der Kuy PHM.

Clinical practice of medication reviews in institutional care settings for older people in the Netherlands: an explorative survey. Eur J Hosp Pharm. 2015;22(4):212-218. 


\section{Chapter 2}

\section{ABSTRACT}

\section{Introduction}

Patient-specific medication reviews might be useful for correcting prescribing omissions. The Dutch Healthcare Inspectorate (IGZ) demands that a medication review is performed by a pharmacist in cooperation with a physician for all residents of nursing homes (twice a year) and residential homes (once a year). This study aims to show the clinical practice of medication reviews for older people in residential and nursing homes by pharmacists. We aimed to answer the following research questions. (i) To what extent are medication reviews performed as required by the IGZ? (ii) How much time is needed to perform the required medication reviews? (iii) In the opinion of the pharmacist, is the medication review economically efficient and medically relevant?

\section{Methods}

A web-based survey was sent to 87 hospital pharmacists and 270 community pharmacists.

\section{Results}

In a 'best case vs worst case' scenario, $76 \%$ vs $42 \%$ of the IGZ-required medication reviews are performed. Considering a mean of 730.7 medication reviews required per year and the mean time spent on one medication review (29.3 min), a pharmacist would need to spend 2.5 months a year to meet the IGZ requirements. Almost every pharmacist considers medication reviews to be medically relevant, but only $47 \%$ consider them to be economically efficient.

\section{Conclusions}

This survey shows that medication reviews performed in institutional care settings do not meet the IGZ requirements, which is not surprising considering the time needed for one medication review. Automation of medication review processes should be initiated to increase efficiency. 


\section{INTRODUCTION}

Elderly patients often have multiple disorders and receive a variety of drugs. Polypharmacy is defined as the concurrent use of multiple drugs. Depending on the definition, this could be more than four, five or nine drugs (1). The Dutch Institute for Public Health and the Environment reported that $30-45 \%$ of the elderly (defined as 65 years or older) use five or more different drugs daily. This percentage is reported to be even higher for patients in residential homes and nursing homes (2).

Polypharmacy and multimorbidity are strongly related. Polypharmacy increases the risk of adverse effects, while adverse effects induce prescribing cascades. The prescribing cascade is the misinterpretation of a drug side effect for a new medical condition, which leads to the prescribing of a new drug (3). In contrast, polypharmacy may induce suboptimal treatment because the probability of underprescription paradoxically increases with the number of drugs used (4). Other consequences of polypharmacy are an increased incidence of drug- drug interactions, drug-disease interactions, nonadherence, and the likelihood of inappropriate prescription $(5,6)$. Using the Beers' Criteria, potentially inappropriate medication is found with a high prevalence of $12 \%$ and $40 \%$ in community-dwelling elderly and nursing home residents, respectively (7). A recent study showed potentially inappropriate prescribing in $60 \%$ and potential prescribing omissions in $42 \%$ of the elderly (8). Inappropriate prescribing increases the incidence of related hospital admissions and should be prevented (9). Patient-specific medication reviews could be useful to correct inappropriate prescribing or prescribing omissions.

\section{MEDICATION REVIEWS}

A medication review is defined as a structured evaluation of the medication of a specific patient, aimed at reaching agreement with the patient, optimising the impact of medicines, and minimising the number of medication-related problems while considering medical history and laboratory values (10). A regular revision of medication increases medication appropriateness while reducing medication related problems. Although effects on primary end points such as hospital admissions have not been proven, it is likely that the frail population of nursing homes and residential homes will benefit from medication reviews (11).

Nursing homes mainly have older residents with chronic somatic diseases or progressive dementia who are not able to perform activities of daily living. A nursing home physician, an officially acknowledged medical discipline, offers medical care to these residents (12). Elderly residents of residential homes are usually less dependent on care. A general practitioner treats elderly residents of these homes. The Dutch Healthcare Inspectorate (IGZ) demands a medication review by pharmacists and physicians for all residents of nursing homes (twice a year) and residential homes (once a 
year). In this review process, the information given by the nursing staff and the patient him/herself should also be taken into account (13). It is unclear to what extent the guidelines of the IGZ regarding the performance of medication reviews are followed in daily practice. This study aims to show residential and nursing homes by pharmacists. Our aim is to answer the following research questions. (i) To what extent are medication reviews performed as required by the IGZ? (ii) How much time is needed to perform the required medication reviews? (iii) Is the medication review economically efficient and medically relevant in the opinion of the pharmacist?

\section{METHODS}

A self-administered web-based survey was developed by three hospital pharmacists and a community pharmacist, who all specialise in performing medication reviews. The survey was piloted on three community pharmacists and two hospital pharmacists, and was adjusted according to their feedback. The data from the pilot study were not included in the present study. The questions were related to (i) pharmacist/ pharmacy characteristics, (ii) cooperation between physicians and pharmacists, and (iii) medication reviews. The survey consisted of 50 questions: 35 multiple choice, 9 numerical, 3 rating scale, and 3 open-ended.

The survey was sent to a total of 357 pharmacies: 87 hospital pharmacies and 270 community pharmacies. The community pharmacies consisted of 30 independent pharmacies and 240 pharmacies belonging to the Mediq-group, which is the market leader of chain pharmacies in the Netherlands. The hospital pharmacies and 30 independent community pharmacies were approached personally, while the chain pharmacies were approached with the aid of the Mediq-group. The survey was closed to answers from pharmacists 6 months after the first invitation had been sent. We received 68 completed surveys. For each pharmacy, only one pharmacist filled in the survey, thus every pharmacist represents one pharmacy organisation. All answers were reported anonymously and could not be linked to a pharmacy. Numerical variables were presented as mean (SD), and n (\%) was used for categorical variables. Although some variables were expected to be positively skewed, means (SD) were used instead of medians (interquartile range) because the mean is more informative in this situation. Descriptive statistics were computed using Microsoft excel 2010.

The medication review can be separated into at least three and as many as six phases: (1) gathering patients' information; (2) pharmacotherapeutic anamnesis; (3) pharmacotherapeutic analysis; (4) creation and determination of the pharmaceutical care plan; (5) determination of the pharmaceutical care plan with the patient; (6) the followup $(14,15)$. For the purpose of this survey, we translated these phases into three steps: preparing (phases 1-3), discussing (phase 4) and finishing (phases 5 and 6) a medication review. The survey also examined the practice of Dutch pharmacists of performing med- 
ication surveillance and the use of a clinical decision support system (CDSS). A system that can provide algorithm-based medication safety alerts electronically is referred to as a CDSS (16). In regular practice, pharmacy information systems use the 'G-standard' to generate medication safety alerts; the G-standard is a nationwide drug database issued by the Royal Dutch Pharmacists Association (KNMP). These medication safety alerts are based on drug-drug and drug-disease interactions which are required to be checked by a pharmacist daily (17).

A newer method for generating additional medication safety alerts involves clinical rules. Clinical rules are algorithms using multiple sources of information such as laboratory values and medication to generate medication safety alerts. If electronic, the clinical rules can be used in a separate monitoring system, but also integrated into the pharmacy information system $(16,18)$.

\section{RESULTS}

The survey was filled in by 68 pharmacists, a response rate of 19\%. Of the respondents, $60 \%$ were practicing in a community pharmacy and $40 \%$ in a hospital pharmacy.

\section{Characterisation of the pharmacists}

Of the 68 pharmacists, $88.2 \%$ offered pharmaceutical services to one type or both types of institution: nursing homes and residential homes. The other pharmacists did not offer pharmaceutical services to these institutions. Table 1 shows that pharmacists offered pharmaceutical services to a mean of 1.0 (SD 1.6) residential home and 2.2 (SD 3.3) nursing homes. The mean number of patients for the pharmacists that offered services was 151.1 (SD 134.0) in residential homes and 555.0 (SD 514.6) in nursing homes. Medication surveillance using the G-standard was used by $98.5 \%$ of the pharmacists.

\section{Cooperation between physicians and pharmacists}

Table 2 shows that $90 \%$ of the pharmacists have recurrent consultations with physicians. In $90 \%$ of the reported cases, these consultations were pharmacotherapy audit meetings (not shown in table 2), which usually occurred monthly (44\%) or quarterly (45\%).

Pharmacotherapy audit meetings occur between pharmacists and physicians (general practitioners or nursing home physicians) to come to an agreement on pharmacotherapy, taking into account national guidelines and the costs of medicines (19). Access to patient information in regular practice (i.e. the indications related to the prescribed drugs and laboratory values) varied greatly between hospital and community 


\section{Chapter 2}

pharmacists. Most of the hospital pharmacists (78\%) had access to laboratory values compared with $29 \%$ of the community pharmacists. The hospital pharmacists with routine access to laboratory values reported that $86 \%$ had access to all values and $14 \%$ to a limited set of laboratory values. A limited set could be renal function only, or also liver function, albumin, electrolytes, cholesterol or the international normalised ratio. Of the community pharmacists with routine access to laboratory values, $25 \%$ had access to all values and $75 \%$ to a limited set, often restricted to kidney function only (67\%). Routine access to the indications for the prescribed drugs was available in $37 \%$ of the hospital pharmacists and $7 \%$ of the community pharmacists.

\section{Medication reviews}

Table 3 shows that most hospital (82\%) as well as community (88\%) pharmacists performed medication reviews (85\% of all pharmacists). Access to laboratory values in regular practice was different from when performing medication reviews; $17 \%$ of pharmacists acquired access to laboratory values when performing medication reviews, and thus $36 \%$ of the pharmacists remained without access. When differentiated, $18 \%$ of hospital pharmacists and $47 \%$ of community pharmacists still lacked access to laboratory values when performing medication reviews. When pharmacists perform a medication review, $67 \%$ reported having access to the indication related to the prescribed drugs. 


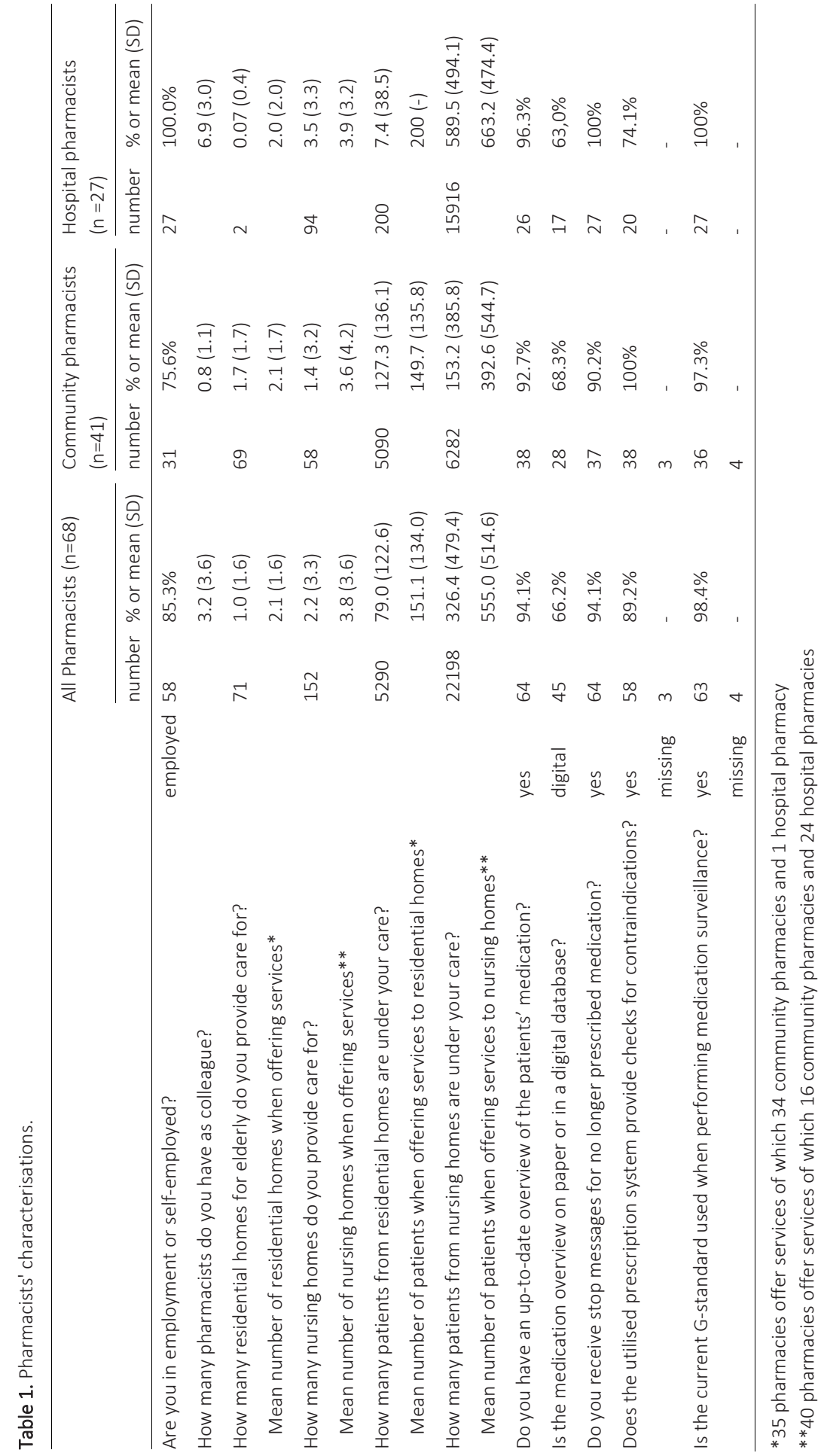


Chapter 2

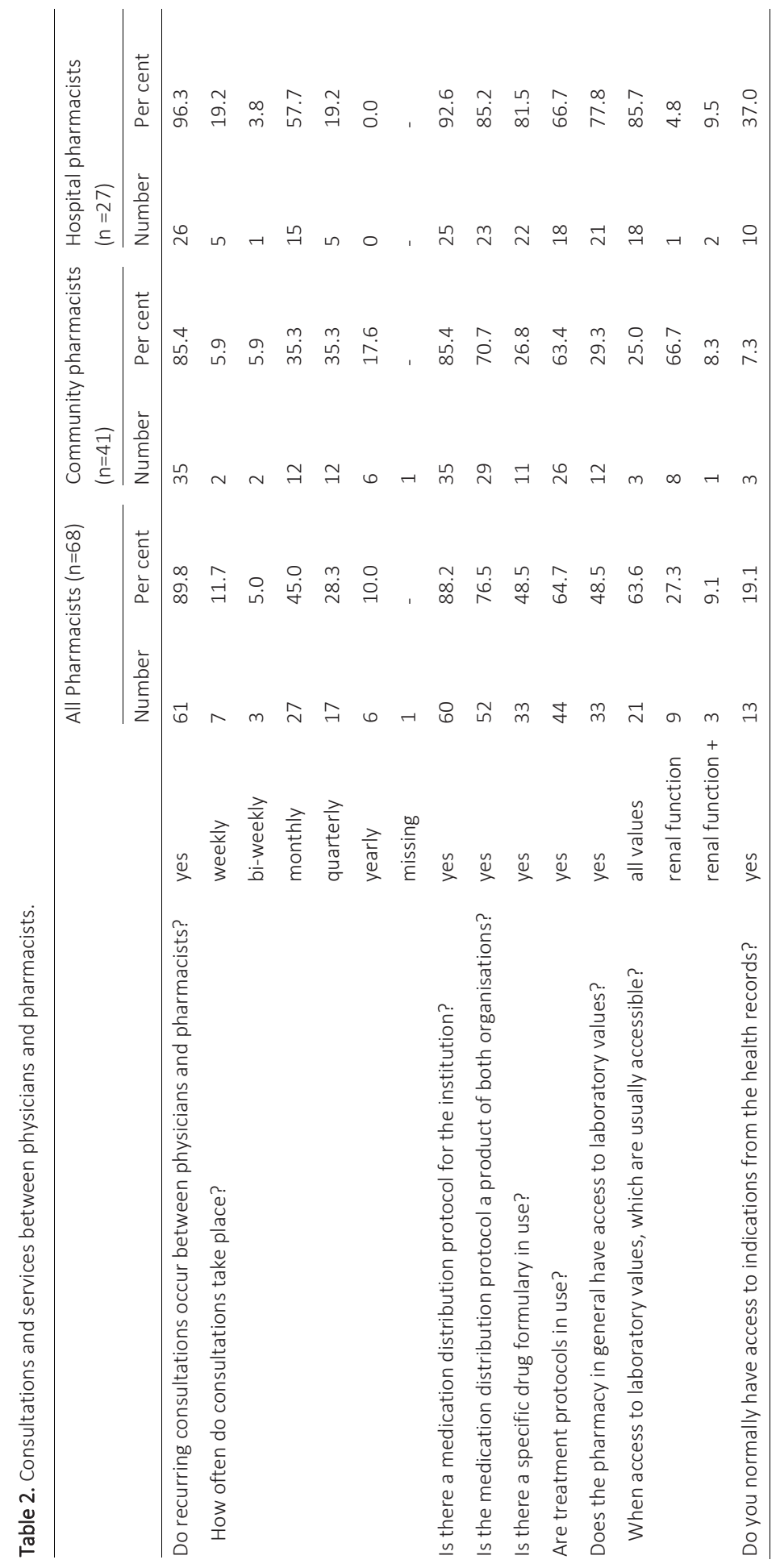


The method most frequently used to support medication reviews involved the use of the pharmacy information system. This was used by $86 \%$ of the pharmacists (data not in table). The use of clinical rules as a support technique for medication reviews was reported by $33 \%$ of the pharmacists. The mean estimated time spent on a medication review for one patient was $29.3 \mathrm{~min}$ (SD 22.0). There was a difference between the mean time spent by community pharmacists (36.6 (SD 24.2)) and hospital pharmacists (20.2 (SD 15.0)). Preparing and finishing the medication review is the main reason for the difference between community and hospital pharmacist. Community pharmacists spent a mean time of 17.7 (SD 11.8) and 15.7 (SD 11.2) min preparing and finishing the medication review, respectively, while hospital pharmacists only spent 9.4 (SD 10.1) and 5.7 (SD 4.8) min.

Eighty-eight per cent of the pharmacists offered services to a total of 27488 patients in any of the above-mentioned institutions, resulting in a mean of 404.2 (SD 506.0) patients for all pharmacists who completed the survey. Eighty-five per cent of all the pharmacists reported performing medication reviews. However, only $89.7 \%$ of the pharmacists reported reviewing a total of 18824 patients yearly. The remaining 10.3\% did not report the number of medication reviews performed. Therefore, the estimated number of medication reviews would be 20996 with a mean of 308.8 (SD 635.3) yearly reviewed patients for all the pharmacists claiming to perform medication reviews.

\section{Pharmacists' opinions}

According to $98 \%$ of the pharmacists, medication reviews are medically relevant. The mean estimated number of interventions suggested by pharmacists in a medication review was 2.0 (SD 1.2), whereas the estimated mean number of interventions accepted by physicians was 1.2 (SD 0.9). Of all the pharmacists, $47 \%$ considered medication reviews to be economically efficient, while $20 \%$ were compensated for performing medication reviews. Almost all pharmacists (98\%) believed that the pharmacist is the right person to perform medication reviews. Almost $13 \%$ of the pharmacists thought that an electronic system could replace the medication review. 


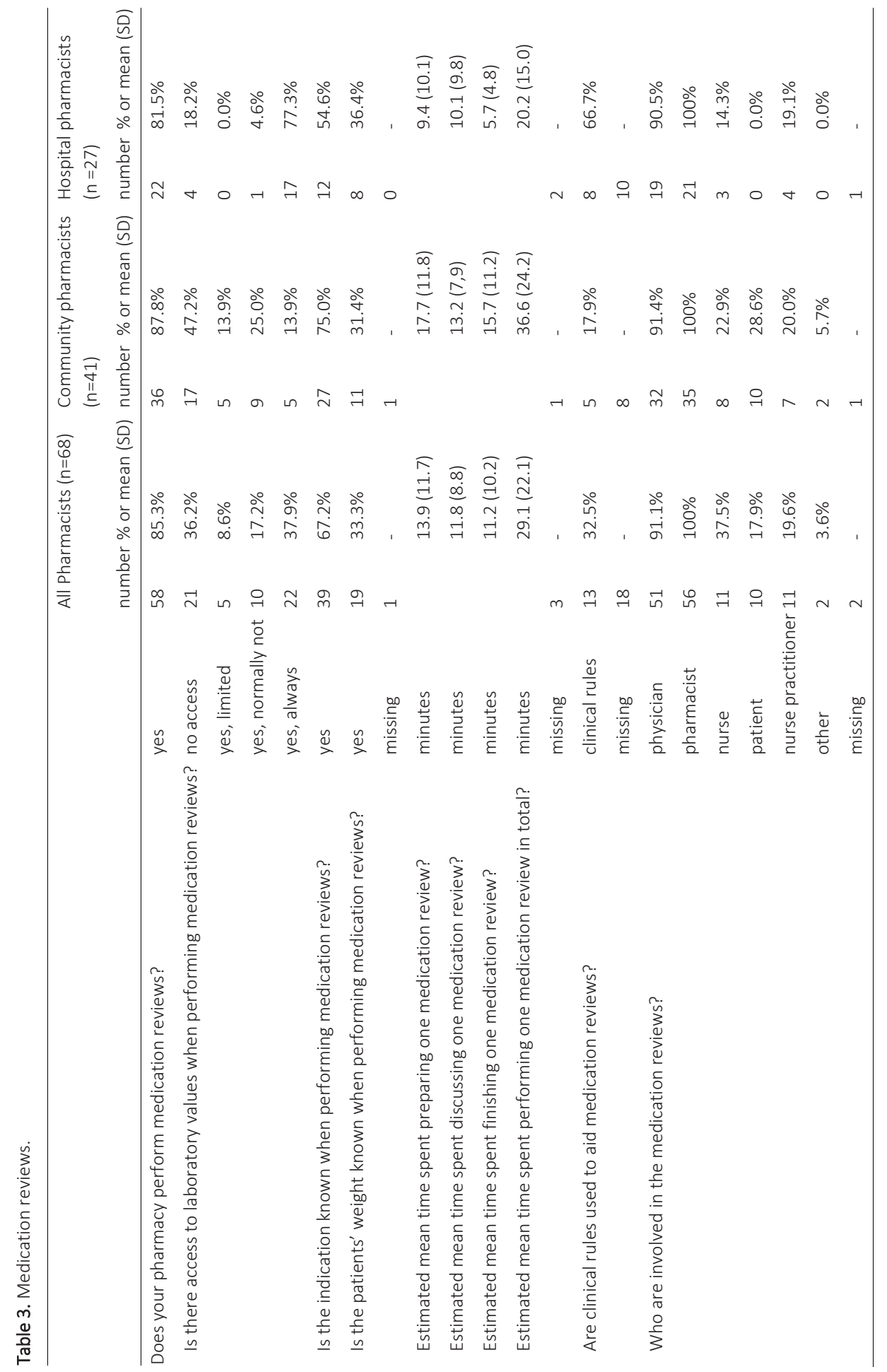


Clinical practice of medication reviews in institutional care settings for older people

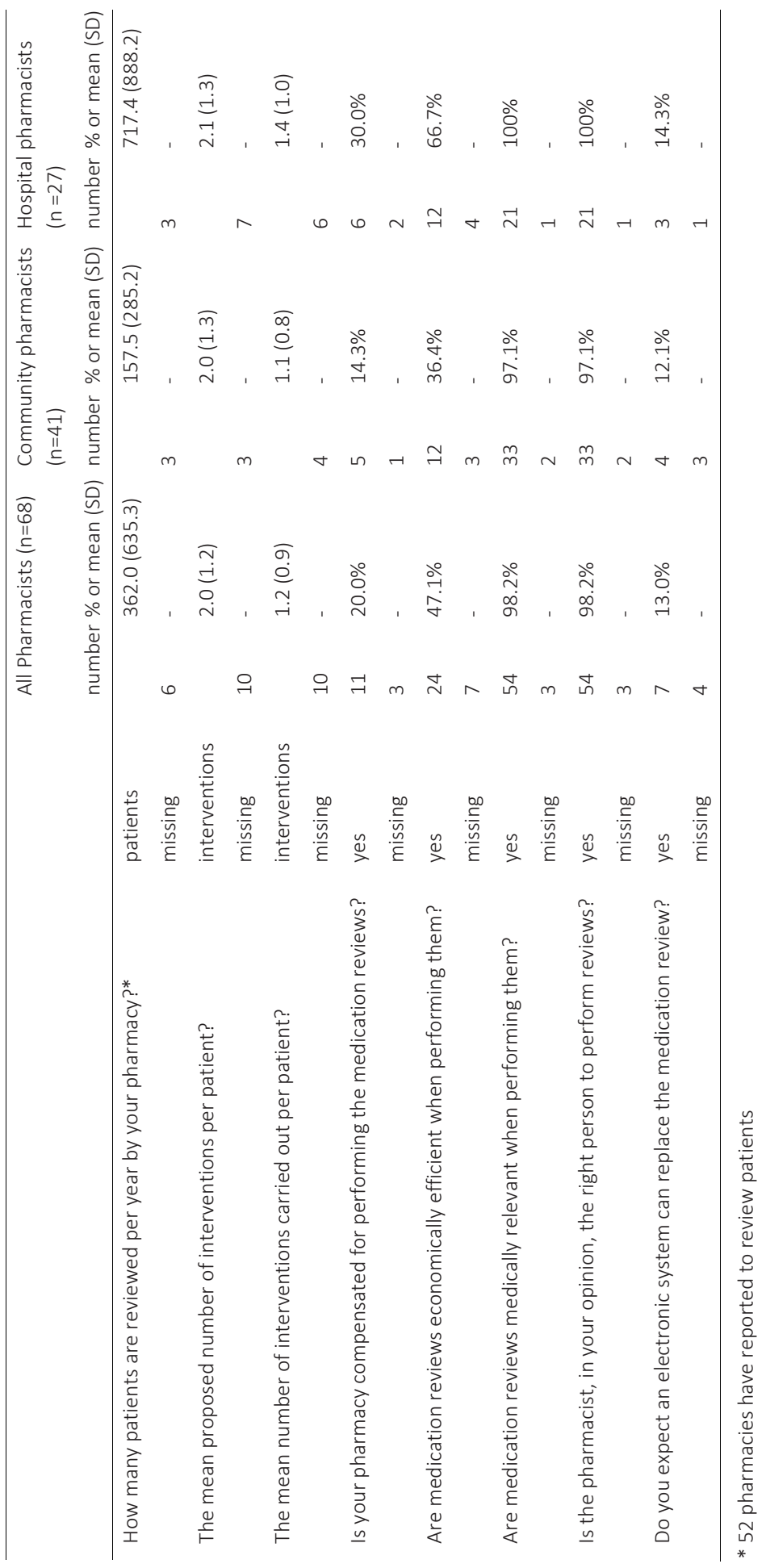




\section{DISCUSSION}

\section{IGZ requirements}

This survey reports on 60 pharmacies that provided care to 27488 patients in nursing homes and homes for the elderly. In 2009, approximately 150000 patients were cared for in such institutions in the Netherlands (20). This survey gives insight into the clinical practice of medication reviews in institutional care settings by pharmacists. To what extent medication reviews are performed according to IGZ requirements can be calculated by taking into account that the IGZ requires patients in residential homes to be reviewed yearly and those in nursing homes to be reviewed twice a year. In this survey, 60 pharmacists offered services to a mean of 88.2 (in total 5290) residential home patients and a mean of 370.0 (in total 22 198) nursing home patients. This results in a total of 49688 yearly IGZ-required medication reviews with a mean of 730.7 per pharmacy. In contrast, the reported annual number of reviewed patients per pharmacy was a mean of 308.8 . This suggests that only $42 \%$ of the IGZ-required medication reviews are performed.

However, the survey reported the number of annually reviewed patients per pharmacy and not the number of medication reviews, meaning that these patients are not necessarily only reviewed once a year. Therefore the calculated 42\% IGZ-required medication reviews performed is a 'worst case' scenario. A comparison of the reported number of patients per pharmacy in a residential or nursing home (404.3) with the reported mean number of patients reviewed (308.8) shows the 'best case' scenario, which is that $76 \%$ of IGZ-required medication reviews are performed.

Although these two percentages vary, it demonstrates that medication reviews are not performed according to IGZ requirements. However, it should be considered that pharmacists select high-risk patients - that is, patients with more than five different medications. Patient selection using a minimum number of medications was one of the inclusion criteria in the Preventing Hospital Admissions by Reviewing Medication (PHARM) Study (14).

\section{Time needed to perform medication reviews}

The time needed to perform the required yearly medication reviews can be calculated using the mean pharmacy size and the amount of time needed by one pharmacist to perform these medication reviews. The 730.7 annually required medication reviews, multiplied by the reported $29.3 \mathrm{~min}$, would take a pharmacist 2.5 months of full-time work a year. A recent report published by the KNMP determined that a regular medication review for patients in an institution such as a nursing home would require $68 \mathrm{~min}$ (15). Taking this into account, one pharmacist would require nearly 6 months of fulltime work annually to perform the IGZ-required medication reviews. The difference 
between the KNMP report and our results should be interpreted with caution and can perhaps be attributed to our approach of simplifying the medication review to three phases. Nevertheless, these results do show that performing IGZ-required medication reviews is very time-consuming, and the amount of time spent by the physician is not even included in this calculation. It is questionable if this amount of time spent on manually performed medication reviews is achievable and acceptable.

The impact on a pharmacy organisation with regard to time needed to perform the required medication reviews will also depend on the number of pharmacists in the organisation. Taking into account the mean number of community and hospital pharmacists (1.8 vs 7.9), the IGZ requirements would have a larger impact on a community pharmacy than on a hospital pharmacy.

\section{Relevance of the medication review}

Pharmacists report that medication reviews are medically relevant, meaning that they are thought to improve the quality of pharmacotherapy. The results show a mean of 2.0 proposed interventions and 1.2 accepted interventions for every medication review. This results in a mean rate of acceptance by physicians of $60 \%$. Other known physicians' acceptance rates vary from $39 \%$ to $91.6 \%$. It has been suggested that physicians' acceptance rate can be correlated with the 'cooperation between physicians and pharmacists', which seemed to be good (90\%) in our results (21). The literature remains ambiguous concerning the effects of manually performed medication reviews in institutions. Medication reviews in nursing home patients do not appear to reduce hospitalisation or mortality, although none of the studies included had the power to detect differences. Another study in care homes also did not show any effect on hospitalisation, mortality and adverse drug events, but did show an increase in medication appropriateness (11).

\section{Automation of the medication review process}

In the KNMP report, the manual acquisition of patient information is an important time factor in the medication reviewing process (15). In our survey, community pharmacists needed to acquire laboratory values more often than hospital pharmacists. This might be the reason why the time spent preparing a medication review was longer for community pharmacists than for hospital pharmacists. One-third of the pharmacists in our study were unable to acquire laboratory data and the indication for the prescribed drugs when performing medication reviews. It has been shown that the absence of laboratory values and indications can influence the outcome of the medication reviews (22). Fortunately, the limited access to indications and laboratory values has recently changed. A new Dutch law states that the reason for prescribing 39 medicines should be stated on the medication order, and, when requested, laboratory values should be given (23). This information is usually retrieved manually, or a 'viewer' application is 
used (15). Automated retrieval of patient information has not yet been incorporated into daily practice. It seems plausible that, when access to indications and laboratory values is automated, the time needed to perform a medication review will decrease.

A recent study has proven the low efficiency of manually performed medication reviews performed by physicians or pharmacists compared with an expert group, suggesting the evident need for a standardised system able to support medication reviews (24). The literature has proven the benefits of CDSS showing improved patient-important outcomes (e.g., adverse drug events) and positive effects on preventive care reminder systems $(25,26)$.

The use of patient information in a CDSS designed to replace the medication review would also require automated access to patient information. This survey reports the scepticism of almost $90 \%$ of the pharmacists about full automation of medication reviews. There is a lack of consensus in the literature also regarding the possibility of a fully automated medication review (27). Besides the necessary laboratory values and indication for the prescribed medication, there is no possibility to electronically collect the required information from the patient or nursing staff. This might be the reason for the pharmacists' scepticism about fully automated medication reviews. Information from the patient or nursing staff is still collected in meetings. A solution to this problem might be the use of self-reported information about an individual's symptoms and perceptions (patient-reported outcomes). Currently, the use of patient-reported outcomes is under investigation for clinical assessments and may be used in the future to facilitate medication reviews (28).

\section{LIMITATIONS}

One of the limitations of this survey is that it was merely piloted in five pharmacists but not validated. However, the consistency of the survey is shown by questions that determined the use of an up-to-date medication overview and the use of the G-standard, since it is expected that all pharmacies in the Netherlands have an up-to-date medication record of their patients and use the G-standard for medication surveillance.

Another limitation of this survey was the low response rate of 19\% (68 pharmacists). Although a response rate of $19 \%$ is low compared to some surveys among pharmacists, in which responses were reported up to $88.6 \%$ in both community pharmacists and hospital pharmacists $(29,30)$, other surveys among Dutch-speaking community pharmacists also showed a low response rate of $7.6 \%$ and $2.9 \%$, respectively $(31,32)$. The low response rate in our study may be the result of the survey-requesting letter. We encouraged pharmacists to complete the survey in relation to nursing and residential homes and to the performance of medication reviews in these long-term care settings. It is possible that many pharmacists who do not perform medication reviews or offer pharmaceutical services to nursing homes or residential homes did not complete the 
survey. Unfortunately, we could not determine whether our survey sample was representative based on specific respondents' characteristics, because these were not determined and it was impossible to retrieve this information since the survey was anonymous. This implies that selection bias may have been introduced. However, the relatively large standard deviations of the respondents' characteristics, suggest an acceptable heterogeneity in the group of respondents. Together with the consistency in their answers, this indicates that the information gathered is sufficiently reliable to answer the research question. However, referring to the aspect of generalisation the results should be interpreted cautiously.

\section{CONCLUSIONS}

This quantitative, explorative survey shows that medication reviews in institutional care settings for older people are not performed as required by the IGZ. We demonstrate that the required medication reviews demand large amounts of time; however, pharmacists do believe that medication reviews are medically relevant. To be able to perform all the IGZ-required medication reviews, more efficient methods are required. A CDSS is able to support medication reviews in a constant and regular way while also reducing the time needed. Automation of the medication review process should be initiated to increase its efficiency. 


\section{REFERENCES}

1. Lin $\mathrm{CF}$, Wang $\mathrm{CY}$, Bai $\mathrm{CH}$. Polypharmacy, aging and potential drug-drug interactions in outpatients in Taiwan: a retrospective computerized screening study. Drugs Aging. 2011;28(3):219-25.

2. Lemmens LC, Weda M. Polypharmacie bij kwestbare ouderen: inventarisatie van risico's en mogelijke interventiestrategieen [Internet]. 2013 RIVM. [ cited on 14 may 2014] Available from: http://www.rivm.nl/Documenten_en_publicaties/Wetenschappelijk/Rapporten/2013/juli/Polyfarmacie_b ij_kwetsbare_ouderen_Inventarisatie_van_risico_s_en_mogelijke_interventiestrategie\%C3\%ABn.

3. Salazar JA, Poon I, Nair M. Clinical consequences of polypharmacy in elderly: expect the unexpected, think the unthinkable. Expert Opin Drug Safety. 2007;6(6):695-704.

4. Cherubini A, Corsonello A, Lattanzio F. Underprescription of beneficial medicines in older people: causes, consequences and prevention. Drugs Aging. 2012;29(6):463-75.

5. Gallagher PF, Barry PJ, Ryan C, Hartigan I, O'Mahony D. Inappropriate prescribing in an acutely ill population of elderly patients as determined by Beers' Criteria. Age Ageing. 2008;37(1):96-101.

6. Shah BM, Hajjar ER. Polypharmacy, adverse drug reactions, and geriatric syndromes. Clin Geriatr Med. 2012;28(2):173-86.

7. Gallagher P, Barry P, O'Mahony D. Inappropriate prescribing in the elderly. J Clin Pharm Therap. 2007;32(2):113-21.

8. Ryan C, O'Mahony D, Kennedy J, Weedle P, Cottrell E, Heffernan M et al. Potentially inappropriate prescribing in older residents in Irish nursing homes. Age Ageing. 2013;42(1):116-20.

9. Leendertse AJ, Egberts AC, Stoker LJ, van den Bemt PM, Group HS. Frequency of and risk factors for preventable medication-related hospital admissions in the Netherlands. Arch Intern Med. 2008;168(17):1890-6.

10. Blenkinsopp A, Bond C, Raynor DK. Medication reviews. Br J Clin Pharmacol. 2012;74(4):573-80.

11. Alldred DP, Raynor DK, Hughes C, Barber N, Chen TF, Spoor P. Interventions to optimise prescribing for older people in care homes. Cochrane Database Syst Rev. 2013;2:CD009095.

12. Schols JM, Crebolder HF, van Weel C. Nursing home and nursing home physician: the Dutch experience. J Am Med Dir Assoc. 2004;5(3):207-12.

13. Dutch Healthcare Inspectorate. Beoordelingskader medicatieveiligheid verpleeghuizen [Internet]. [accessed on 01-09-2011]. Available from: http://www.igz.nl/Images/Bijlagen\%20Beoordelingskaders\%20 en\%20Risicoscore\%20bij\%20rapport\%20Medicatieveiligheid_tcm294-287277.pdf.

14. Leendertse AJ, de Koning FH, Goudswaard AN, Jonkhoff AR, van den Bogert SC, de Gier HJ et al. Preventing hospital admissions by reviewing medication (PHARM) in primary care: design of the cluster randomised, controlled, multi-centre PHARM-study. BMC Health Serv Res. 2011;11:4.

15. Significant. Generieke kosten medicatiebeoordeling [Internet]. 2014. [cited on 1 Sep. 2014] Available from: http://www.knmp.nl/downloads/nieuws/downloads-nieuws-2014/SignificantRapportGeneriekekosten medicatiebeoordelingDefinitief.pdf.

16. Eppenga WL, Derijks HJ, Conemans JM, Hermens WA, Wensing M, De Smet PA. Comparison of a basic and an advanced pharmacotherapy-related clinical decision support system in a hospital care setting in the Netherlands. J Am Med Inform Assoc. 2012;19(1):66-71.

17. KNMP. Handboek medicatiebewaking [Internet]. 2013. [cited on 20 Nov. 2015] Available from: https://www.knmp.nl/patientenzorg/medicatiebewaking/handboek-medicatiebewaking-2013.

18. Rommers MK, Teepe-Twiss IM, Guchelaar HJ. A computerized adverse drug event alerting system using clinical rules: a retrospective and prospective comparison with conventional medication surveillance in the Netherlands. Drug Saf. 2011;34(3):233-42.

19. Teichert M, van der Aalst A, de Wit H, Stroo M, De Smet PA. How useful are prescribing indicators based on the DU90\% method to distinguish the quality of prescribing between pharmacotherapy audit meetings with different levels of functioning? Eur J Clin Pharmacol. 2007;63(12):1171-7.

20. Centraal Bureau voor de Statistiek. Open database [Internet]. [cited on 2 Dec. 2014]. Available from: www.cbs.nl. 
21. Verrue CL, Petrovic M, Mehuys E, Remon JP, Vander Stichele R. Pharmacists' interventions for optimization of medication use in nursing homes : a systematic review. Drugs Aging. 2009;26(1):37-49.

22. Mestres Gonzalvo C, Hurkens KPGM, de Wit HAJM, Janknegt R, Schols JMGA, Mulder WJ et al. Covariates that influence the quality of a medication review. Eur J Hosp Pharm. 2013;0:1-4

23. Staatscourant.Wijziging regeling der geneesmiddelenwet [Internet]. [cited on 20-08-2014]. Available from: https://zoek.officielebekendmakingen.nl/stcrt-2011-22913.html.

24. Mestres Gonzalvo C, Hurkens KP, de Wit HA, van Oijen BP, Janknegt R, Schols JM et al. To what extent is clinical and laboratory information used to perform medication reviews in the nursing home setting? the CLEAR study. Ther Clin Risk Manag. 2015;11:767-77.

25. Tawadrous D, Shariff SZ, Haynes RB, lansavichus AV, Jain AK, Garg AX. Use of clinical decision support systems for kidney-related drug prescribing: a systematic review. Am J Kidney Dis. 2011;58(6):903-14.

26. Jaspers MW, Smeulers M, Vermeulen H, Peute LW. Effects of clinical decision-support systems on practitioner performance and patient outcomes: a synthesis of high-quality systematic review findings. J Am Med Inform Assoc. 2011;18(3):327-34.

27. de Wit HA, Mestres Gonzalvo C, Janknegt R, Schols JM, van der Kuy PH. A fully automated medication review? Int J Clin Pharm. 2014;36(2):220-1.

28. Bevans M, Ross A, Cella D. Patient-Reported Outcomes Measurement Information System (PROMIS): Efficient, standardized tools to measure self-reported health and quality of life. Nurs Uutlook. 2014;62:339-45.

29. van der Veen W, de Gier HJ, van der Schaaf T, Taxis K, van den Bemt PM. Risk analysis and user satisfaction after implementation of computerized physician order entry in Dutch hospitals. Int J Clin Pharm. 2013;35(2):195-201.

30. Florentinus SR, van Hulten R, Kramer M, van Dijk L, Heerdink ER, Leufkens HG et al. Which pharmacists contribute to high-level pharmacotherapy audit meetings with general practitioners? Ann Pharmacother. 2006;40(9):1640-6.

31. Patel M, Slack M, Cooley J, Bhattacharjee S. A cross-sectional survey of pharmacists to understand their personal preference of brand and generic over-the-counter medications used to treat common health conditions. J Pharm Policy Pactice. 2016;9:17.

32. Van Bever E, Elseviers M, Plovie M, Vandeputte L, Van Bortel L, Vander Stichele R. Attitudes of physicians and pharmacists towards International Non-proprietary Name prescribing in Belgium. Basic Clin Pharmacol Toxicol. 2015;116(3):264-72. 



\section{Chapter}

\section{Evaluation of clinical rules in a standalone pharmacy based clinical decision support system for hospitalised and nursing home patients}

This chapter is based on:

de Wit HAJM, Mestres Gonzalvo C, Cardenas J, Derijks HJ, Janknegt R, van der Kuy PHM, Winkens B, Schols JMGA.

Evaluation of clinical rules in a standalone pharmacy based clinical decision support system for hospitalized and nursing home patients. Int J Med Inform. 2015;84(6):396-405. 


\section{Chapter 3}

\section{ABSTRACT}

\section{Introduction}

In the Zuyderland Medical Centre a home-grown standalone advanced clinical decision support system (CDSS) is used for medication surveillance. The aim of this study is to determine the efficiency of this CDSS and to identify and quantify the benefits and limitations of the system allowing the improvement the CDSS.

\section{Methods}

Alerts and handling of the executed clinical rules were extracted from the CDSS from the period September 2011 to December 2011. The number of executed clinical rule alerts, number of actions on alerts, and the reason why alerts were classified as not relevant were analyzed. The alerts where considered clinically relevant when the pharmacist needed to contact the physician.

\section{Results}

The 4065 alerts have been separated into; 1137 (28.0\%) new alerts, 2797 (68.8\%) repeat alerts and 131 (3.2\%) double alerts. When the alerts were analyzed, only $3.6 \%$ were considered clinically relevant. Reasons why alerts were considered as not to be relevant were: a) the dosage was correct or already adjusted, b) the drug was (temporarily) stopped, c) the monitored laboratory value or drug dosage was already reverted to within the reference limits. The reasons for no action were linked to three categorical limitations of the used system; 'algorithm alert criteria', 'CDSS optimization', and 'data delivery'.

\section{Conclusions}

This study highlighted a number of ways in which the CDSS could be improved. These different aspects have been identified as important for developing an efficient CDSS. 


\section{INTRODUCTION}

Prescription errors have been defined as 'transcription errors', 'failure to communicate essential information' and 'the use of drugs or doses inappropriate for the individual patient' (1). Prescription errors can lead to adverse drug events and several studies have shown that adverse drug events (ADEs) can cause hospital admissions (2). The HARM-study showed that half of all hospital admissions due to ADEs are preventable (3). Prevention of prescription errors and related adverse drug events is one of the primary tasks of pharmacists in The Netherlands. This involves checking of prescriptions for key issues such as drug-drug interactions, drug contra-indications and dosage. This can also be referred to as 'medication surveillance' (4). The introduction of computerized physician order entry (CPOE) systems in hospital settings has been shown to reduce prescription errors and $\operatorname{ADES}(5,6)$. Khajouei et al. have shown that CPOE has a beneficial impact with positive effects on efficiency, workflow and medication safety for endusers, however operational deficiencies have also been reported such as lack of allergy alerts and integration with other hospital systems (7). Medication surveillance can be automated by the use of algorithms, also referred to as clinical rules, which enables the creation of drug safety alerts. These clinical rules exist in varyingly complexity (8). A system that can execute algorithm based clinical rules is referred to as a (computerized) clinical decision support system (CDSS). Many studies have shown the benefits of using a CDSS for healthcare providers including the generation of drug safety alerts for medication surveillance $(9,10)$.

In The Netherlands drug safety alerts include drug-drug interactions, drug contraindications and dosage appropriateness and are generated by the so-called 'Gstandard', a nationwide drug database maintained by the Royal Dutch Pharmacists Association (KNMP). The algorithms generating drug-drug interactions and dosage alerts are relatively to construct. In addition, contra-indication alerts are generated if contra-indications and allergies are registered within the CPOE system. The Dutch Healthcare Inspectorate (IGZ) requires physicians to prescribe exclusively using CPOE systems with integrated drug safety alerts from the 'G-standard' to ensure robust medication surveillance (11).

The use of algorithm based clinical rules that combine medication and laboratory values is not yet common practice in The Netherlands. Further, clinical rules using laboratory values are not (yet) integrated into CPOE and consequently are restricted to pharmacists using standalone CDSS.

There are studies showing the benefits of algorithm based clinical rules using multiple data sources. Kane-Gill et al. showed that such clinical rules can detect adverse drug reactions in the ICU (12). Tawadrous et al. showed, in a systematic review, that such clinical rules have a beneficial effect on renal function related drug prescribing (13). In total, 32 studies were examined of which 17 (53\%) involved computerized CDSSs. Eleven of the 17 (65\%) computerized CDSSs studies reported statistically significant im- 
provements in clinician prescribing outcomes (e.g. frequency of appropriate dosing). Patient-specific outcomes (e.g. adverse drug events) were considered in seven computerized CDSS studies, with two of these resulting in significant improvements. These two studies involved the variability of hemoglobin levels from target range and the decreased development of renal impairment $(14,15)$.

The implementation of guidelines by using clinical rules, such as the signaling of gastrointestinal prophylaxis in patients at risk, is another approach to medication surveillance. Some hospitals use clinical rules to prevent adverse drug events from nonsteroidal anti-inflammatory drugs (NSAIDs) in at risk patients. Rommers et al. showed that such clinical rules can prevent adverse drug events when used in combination with the normally available 'G-standard'. They reported a difference for the number of interventions made by a hospital pharmacist between the sole use of G-standard and the use of the G-standard augmented by clinical rules in a standalone CDSS (3.7\% vs. 19.4\%) which included a NSAID gastrointestinal prophylaxis clinical rule (8). The drug safety alert for gastrointestinal prophylaxis highlights the difference between algorithms used in the G-standard and clinical rules using multiple sources of information. In the Gstandard an alert will be generated when a patient uses a NSAID in combination with an antiplatelet aggregation drug to ensure the prescription of gastrointestinal prophylaxis. However, this alert needs to be manually reviewed in case gastrointestinal prophylaxis has already been prescribed. A standalone CDSS will generate a list of patients at risk which is dependent on specific drug combinations, age, co-medication, and either the absence of gastrointestinal prophylaxis, or a low dose of gastrointestinal prophylaxis.

The lower the clinical relevance of any alert, the higher the risk of alert fatigue by users. Alert fatigue occurs when there is a high number of non-clinically alerts, thereby resulting alerts both relevant and not-relevant being ignored. Drug safety alerts are reported to be ignored by physicians in $49 \%$ to $96 \%$ of the occasions (16). To reduce alert fatigue a structural assessment of the algorithms in the G-standard is made by taking into account four parameters: i) evidence relating to the interaction; ii) clinical relevance of the potential adverse reaction resulting from the interaction; iii) risk factors identifying patient, medication or disease characteristics for which the interaction is of particular importance; and iv) the incidence of the adverse reaction (17). Despite the structural assessment of clinical relevance the G-standard still has a low percentage of relevant drug safety alerts (5.8\%), however this has been shown to increase to $17 \%$ when used in combination with a standalone CDSS (18). In relation to alert fatigue, more complex clinical rules can in some cases replace simple G-standard algorithms thereby reducing the number of alerts. However, most of the more complex clinical rules will also be complementary and thereby generate additional alerts.

In Zuyderland Medical Centre, the Department of Clinical Pharmacy and Toxicology, has a home-grown standalone CDSS based on a Microsoft Access database. The CDSS has been implemented in both a hospital and nursing home setting with 450 and 800 beds, respectively. The CDSS is used on a single site by eight pharmacists for medication 
surveillance. It is used in combination with the 'G-standard' which is integrated in the Vipharma CPOE system. The CDSS operates by extraction of the medication data from the admitted patients and by extraction of the laboratory data from all admitted patients two weeks prior to the extraction date. Medication data is extracted from the CPOE by using the business intelligence application 'Crystal Reports'. Laboratory data is obtained daily from the laboratory by means of an electronic file. Both files are loaded into the Access database, see figure 1.

Since 2008, thirty-nine clinical rules have been created in the standalone CDSS. The clinical rules were mainly derived from the 'informatorium medicamentorum' issued by the KNMP but also known ADEs or practical prescribing mistakes were included to augment the CPOE integrated G-standard clinical rules (19-21). Whenever a laboratory value is below or above a set limit, in combination with the appropriate drug, the system will generate an alert. The clinical rules cover dosage adjustments in decreased renal function, electrolyte dysfunction in relation to medication, the determination of drug levels in presence of both hypoalbuminemia and leucocytosis, stop-dates of oncolytics, and specific dosages such as weekly methotrexate.

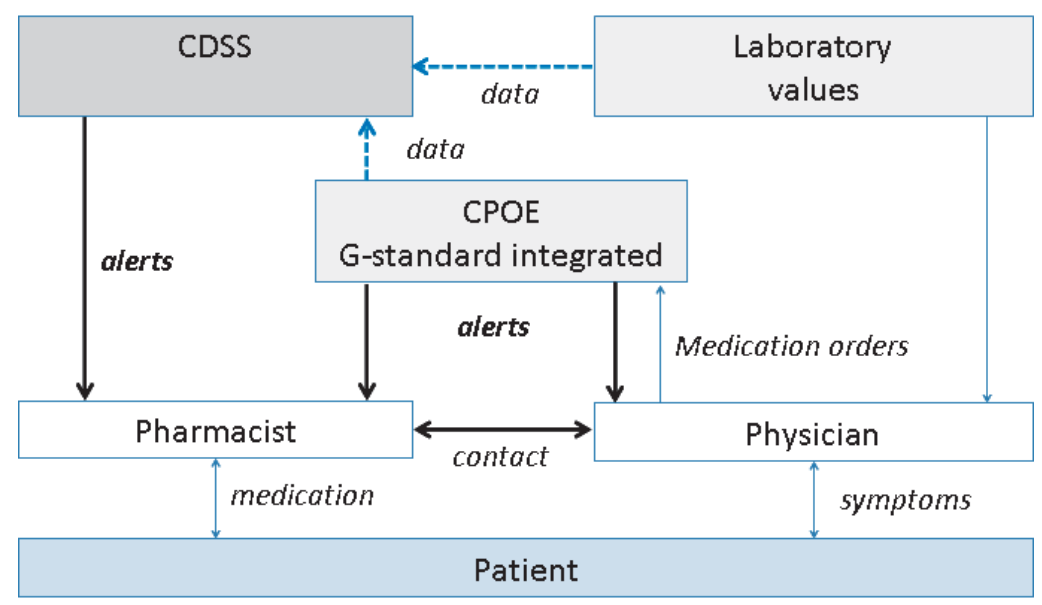

Figure 1. the medication loop positions the standalone CDSS. The alerts of the CDSS are only reviewed by pharmacists. If necessary the physician is contacted. The CPOE integrated G-standard generates alerts which can be reviewed by both pharmacists and physicians.

Checking of critical dosages and stop-dates has to be done manually. Only the prescription of an oncolytic or an out of range laboratory value is the trigger for an alert; drug dosage itself is not a trigger. The cut-off value for the laboratory values to create an alert are: eGFR-MDRD $<60 \mathrm{ml} / \mathrm{min}$, sodium $<130 \mathrm{mmol} / \mathrm{l}$, potassium $>5.5$ or $<3.0$ $\mathrm{mmol} / \mathrm{I}, \mathrm{INR}>5$, Leucocytes $<4 \times 10^{9} /$. This system has brought benefits by identification of the need for relevant medication interventions, however there are also negative effects due to the level of irrelevant alerts. The aim of this study was to identify and 
quantify both the positive and negative effects of the clinical rules in the current CDSS. This quantification will enable the efficiency of the CDSS to be assessed as well as yielding information on how it can be improved.

\section{METHODS}

This study was conducted at the Department of Clinical Pharmacy and Toxicology of Zuyderland Medical Centre. The study did not require ethical approval since it involved the retrospective analysis of a database used for standard care. Drug safety alerts and their management were extracted from the CDSS in the period. September 2011 to December 2011. A period of 4 months was chosen to limit the number of alerts to be analyzed. Alerts that were generated in August, but were repeated in the study period were excluded. The data were selected in such a way that it was possible to distinguish in the clinical rule alerts between: 'new alerts', 'repeat alerts' and 'double alerts'. A new alert was a clinical rule seen for the first time in a selected patient. A repeat alert was the same clinical rule reappearing daily, already evaluated by the pharmacist. Finally, a double alert was a clinical rule with the same content that occurred twice in the same patient on the same day. Evidently, when an alert reappeared daily the probability that action is needed will be lower than with new alerts since repeat alerts would already have been evaluated. The pharmacist evaluates the alert, for which the electronic health record (EHR) can be consulted, and subsequently the pharmacist advises the physician according to the relevant guidelines. The guidelines used to create the clinical rules have been developed by the pharmacists and physicians within Zuyderland Medical Centre. Nevertheless, in some cases the physician intentionally deviates from these guidelines taking into account other patient specific factors. Therefore, alerts were considered as clinically relevant whenever the pharmacist contacted the physician, irrespective of the outcome of the contact between the physician and pharmacist. The relevance of the alerts was determined by checking whether or not the pharmacist contacted the physician for each alert (an alert with an action). At the point of alert evaluation the pharmacist was obliged to register the reason for an 'action' or 'no action' (where an action results in a consultation between pharmacists and physicians). In addition, the appearance of an alert on two occasions was documented. The appropriateness of the pharmacist's evaluation was not validated in this study, but the pharmacist's documented reason following the identification of the alerts is already being assessed by another pharmacist.

The extracted alerts were analyzed by two pharmacists. One pharmacist, not trained in evaluating the alerts in practice, counted the pharmacist's documented alert evaluations and when the documented alert evaluation was unclear the second pharmacist trained in evaluating the alerts was consulted. The clinical rule efficiency (CRE) was defined as the percentage of clinically relevant alerts per clinical rule, i.e. the total num- 
ber of clinically relevant alerts divided by the total number of alerts. The new alert efficiency (NAE) and the repeat alert efficiency (RAE) was the efficiency of the clinical rules when appearing for the first time in a selected patient and when reappearing, respectively. The number of actions for both new alerts and repeat alerts was divided by the total number of new and repeat alerts to calculate the NAE and RAE, respectively. The total efficiency of the CRE, NAE and RAE was defined as the percentage of the total of clinically relevant alerts in each group in comparison with the total number of alerts in each group.

\section{RESULTS}

In the selected four months a total of 4652 alerts were generated from 900 individual patients and registered in the Access database. Thirty-five out of thirty-nine (90\%) clinical rules have been analysed, see table 1 for the selected clinical rules. The four clinical rules not selected for analysis involved rules not used for medication surveillance. Out of the 4652 alerts generated 587 alerts were not selected for analysis because they had been initiated before the selected period and were repeated in the study period. The 4065 analyzed alerts were generated over eighty-three days corresponding to an average of 48.5 alerts daily.

\section{Efficiency of the alerts}

The 4065 alerts were grouped into categories; 1137 (28.0\%) new alerts, 2797 (68.8\%) repeat alerts and 131 (3.2\%) double alerts.

Out of 1137 new alerts and 2797 repeat alerts, 123 (NAE 10.9\%) and 24 (RAE 0.9\%) required action, respectively. The efficiency of all clinical rules was 147 out of 4065 (CRE $3.6 \%)$. See figure 2.

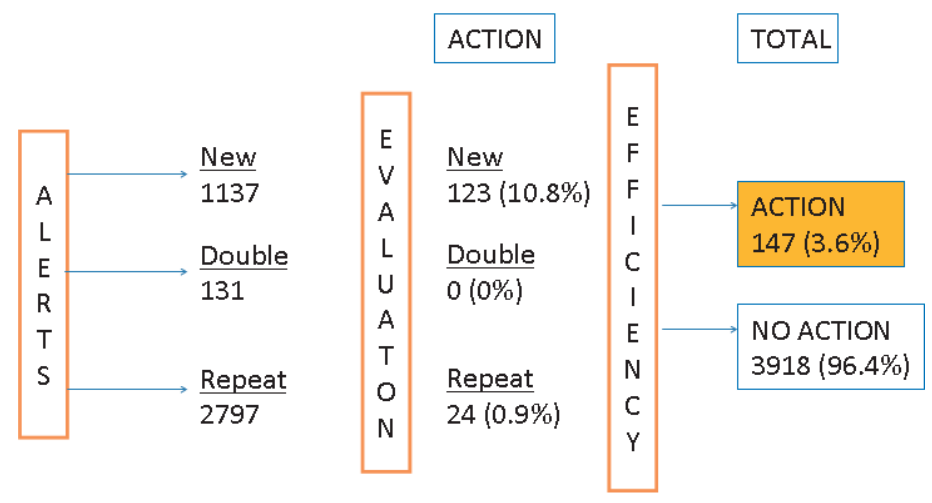

Figure 2. The efficiency of different CDDS alerts. 
Chapter 3

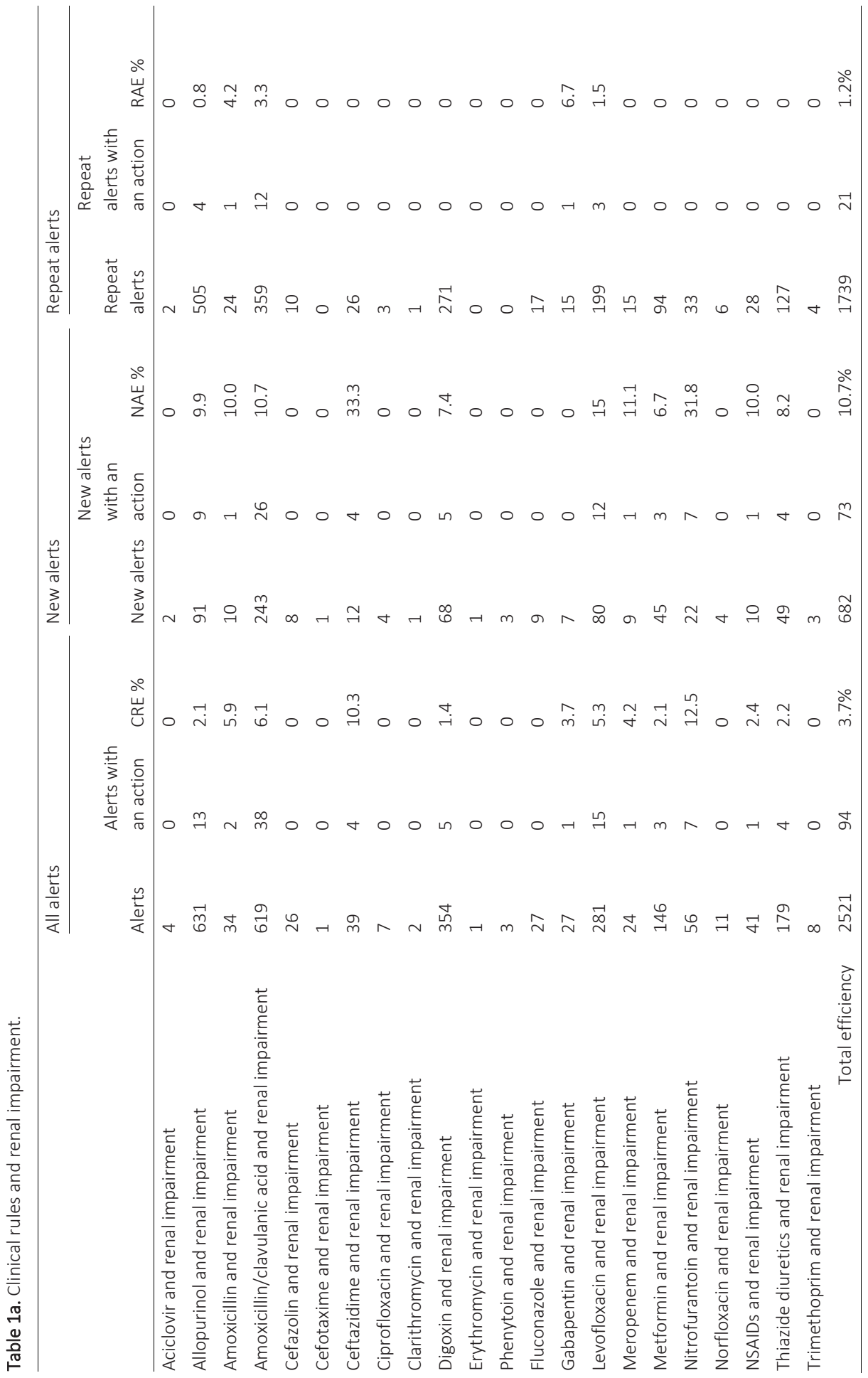


Evaluation of clinical rules in a standalone pharmacy based CDSS

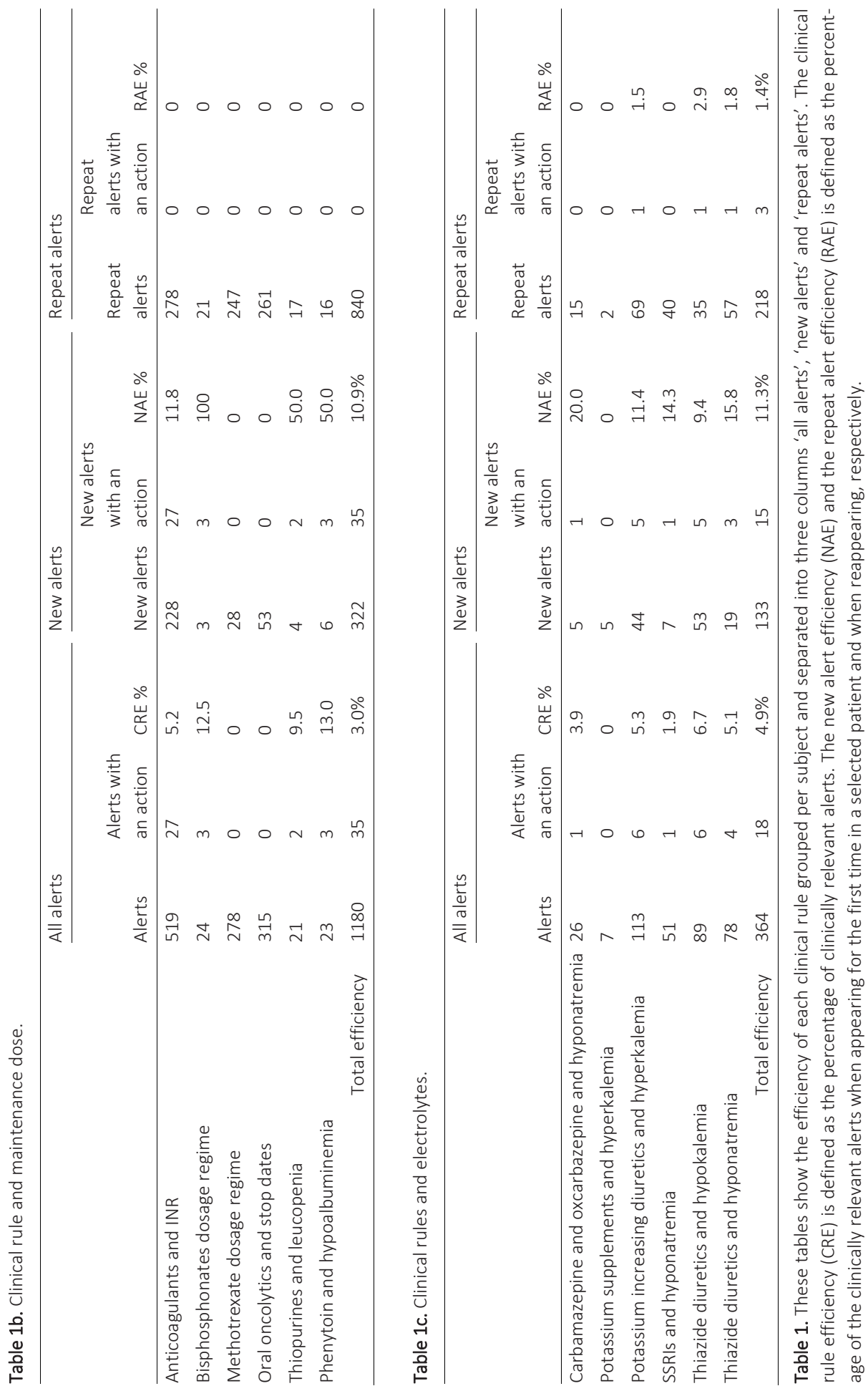




\section{Chapter 3}

The efficiency and number of alerts for each separate clinical rule is shown in Table 1. The second column in Table 1 shows the CRE for each separate clinical rule. The clinical rules in Table 1 are divided into three main groups: clinical rules for impaired renal function, clinical rules for maintenance dose and clinical rules for electrolyte imbalances.

Only a few clinical rules: 'phenytoin with hypoalbuminemia', 'bisphosphonates dosage regime' and 'ceftazidime with decreased renal function', had an efficiency of over $10 \%$.

Most of the clinical rules showed efficiencies lower than $10 \%$ and in some instances $0 \%$ as seen in the clinical rules 'oral oncolytics and stop dates' and 'methotrexate dosage regime', respectively.

Comparing the CRE with the NAE demonstrated that the NAE was much higher than the CRE for all clinical rules (10.9\% vs. 3.6\%). Almost all clinical rules would increase in efficiency when only the first alert was taken into account.

\section{Reasons for no action}

The appearance of double alerts (3.2\% of the total number of alerts) was a reason for both no actions and a lower efficiency. Double alerts mostly appeared because a drug was being prescribed with a 'loading dose' and a 'maintenance dose' as separate medication orders. In order to optimize the system and also as to the reasons why alerts were triggered but did not require any actions have been investigated. This was done by analyzing four different clinical rule categories.

Figure 3 shows the reasons why no action was needed for the categories: 'impaired renal function', 'elevated INR', 'electrolyte imbalance', and 'maintenance dose'. For each category the main reason as to why the clinical rules were interpreted as not relevant by the pharmacist was different.

The main reason an action was not required in the category 'impaired renal function' (figure 3a), was because the dosages were correct in $78 \%$ of the alerts. For the category 'elevated INR', which was a clinical rule monitoring the INR in coumarin usage (figure $3 \mathrm{~b}$ ), an action was not required in $61 \%$ of the alerts because the coumarin dosage was already adjusted by the physician based on the elevated INR. The category 'electrolyte imbalance', consisting of clinical rules triggered when electrolytes are influenced by drug therapy (figure $3 c$ ), did not require an action because the drug was already (temporarily) stopped in $47 \%$ of the alerts. Other reasons for the absence of actions in all categories were: the monitored laboratory value had already improved to within the reference limits, and the clinical parameter was (already) right on reference limit and only observation by the pharmacist was required, or the patient had already been discharged from the hospital.

The efficiency of the clinical rules category associated with maintenance dose 'oral oncolytics and stop dates' and 'methotrexate dosage regime' was $0 \%$. Many alerts were generated by these clinical rules but no actions were needed simply because both the stop dates and dosage regimes were always correct. In contrast, a similar clinical rule 
category 'bisphosphonates dosage regime' demonstrated a CRE of $12.5 \%$ and a NAE of $100 \%$, respectively.

\section{Categorical limitations}

The reasons for no action vary greatly and are linked to limitations of categories used in the system. As shown in figure 3a. the reason for no action on alerts was because the dosage was often correct. The alerts with no action were generated without taking into account the prescribed dosage but merely based on lowered eGFR-MDRD. This can be related to the limitation of the category 'algorithm alert criteria' (see figure 4a). Another example of the importance of the algorithm alert criteria was demonstrated with the clinical rules 'methotrexate dosage regime' and 'bisphosphonates dosage regime'. In the case of the first clinical rule the pharmacist was obligated to manually check the dosage of the methotrexate in the CPOE. In the case of the latter clinical rule, the algorithm also takes the dosage regime into account and therefore only generated an alert when the bisphosphonate dosage deviated from the normal prescribed dosage. The difference in algorithm construction was confirmed by the difference in CRE (0\% vs. $12.5 \%$ ), NAE (0\% vs. $100 \%$ ) and the number of irrelevant alerts (278 vs. 21 ). The algorithm 'bisphosphonates dosage regime' therefore is more efficient.

Another limitation of the categorisation can be seen from figure 2 which shows the high level of already evaluated alerts, which can be linked to the limitations of the CDSS. The way the CDSS is constructed will cause the alerts to reappear, whilst the data does not change.

Finally, the occurrence of 131 double alerts (3.2\%) shown in figure 2 and the clinical rules that were evaluated as irrelevant because the laboratory value had already changed to normal reference limits shown in figure $3 \mathrm{~b}$ and $3 \mathrm{c}$ can both be linked to the defined category 'data delivery' to the CDSS. The double alerts are a result of inadequate data delivery while the determination of laboratory values and change to normal reference limits is a consequence of the delay in data delivery thereby leading to more unnecessary alerts. 

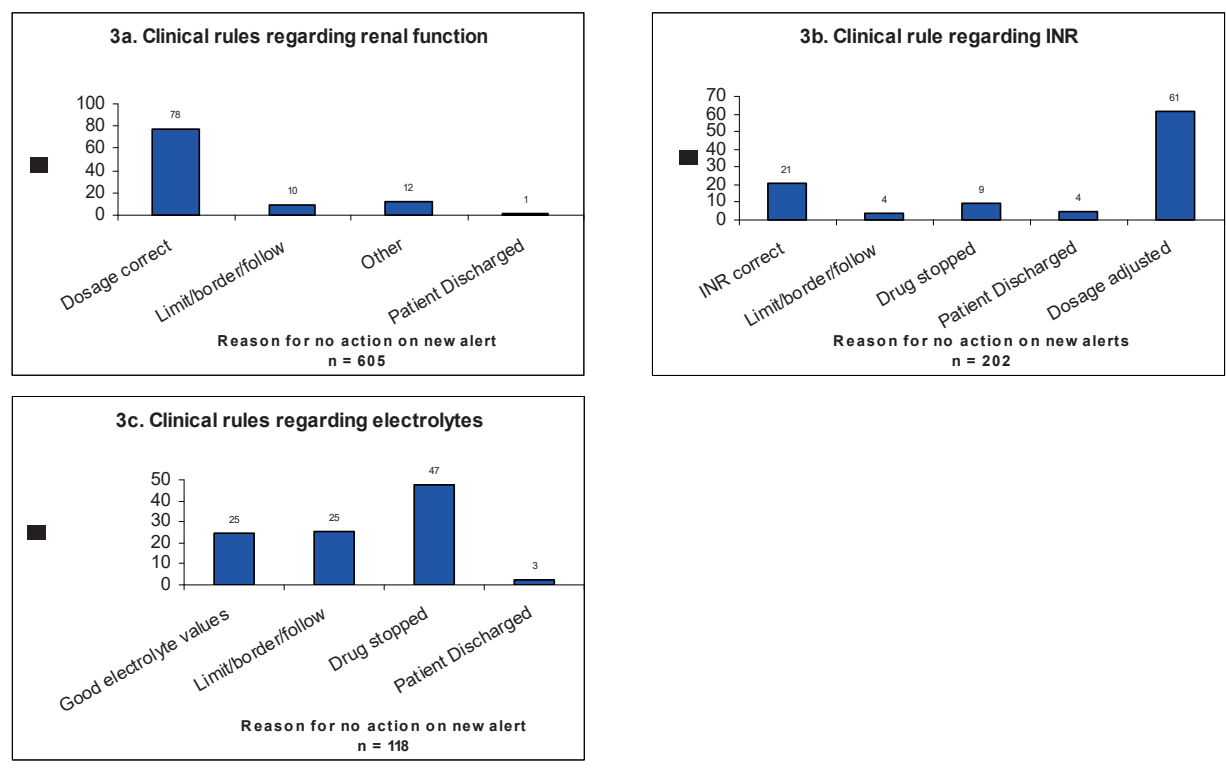

Figure 3. Shows for three categories the reasons why no action was required when an alert appeared. Figure 3a. Shows the clinical rules associated with impaired renal function. Figure 3b. With an elevated INR. Figure 3c. With electrolyte imbalance.

\section{DISCUSSION}

This study shows that the 35 clinical rules in the Zuyderland Medical Centre have a small efficiency of $3.6 \%$. The reported number of irrelevant alerts may lead to alert fatigue and more efficient CDSSs with complex clinical rules have been reported $(16,18)$. Nevertheless, it has been shown that a standalone CDSS can be (relatively easily) developed in a straightforward business intelligence system thereby augmenting the regular medication surveillance for pharmacists. The alerts generated are specific for high risk prescriptions and at-risk patients regarding renal function or other laboratory deviations in relation to prescribed medication.

The use of these more complex clinical rules, in addition to the CPOE integrated Gstandard, should be encouraged since it augments regular medication surveillance. When considering the development or implementation of a CDSS with more complex clinical rules for medication surveillance the three category limitations influencing efficiency should be addressed.

\section{Algorithm alert criteria}

The first category; the algorithm alert criteria should optimally also consider the dosage and specified eGFR-MDRD boundaries. This can be done by specifying the algorithm 
alert criteria. If the algorithm is specified according to the prescribing guideline in renal impairment then alerts will only appear when the prescribed dosage is above or below the dosage limits for the specified renal function. For instance, the algorithm should not show an alert when intravenously administered amoxicillin/clavulanic acid 1000/200mg is prescribed twice daily with an EGFR-MDRD of $24 \mathrm{ml} / \mathrm{min}$. However, when the dosage is three times daily, an alert should be generated. By adding the limits of dosage frequencies corresponding with decreased renal function boundaries to the algorithm alert criteria this may greatly increase the efficiency of the clinical rule (see figure 4b).

Further, the clinical rules regarding the checking of critical prescriptions such as stop dates of oral oncolytics can be improved by algorithm adjustments. The reason for the absence of actions in the clinical rules for oral oncolytics is that most oral oncolytics in the hospital are used for long periods of time but with limited dosage variations. These oncolytic dosages should be integrated in the alert criteria of the algorithms.

Additionally, the results in figure $3 b$ show that clinical rules are sometimes not evaluated as relevant because the values presented in the clinical rule are at the limit for relevance. For example, a sodium concentration is $129 \mathrm{mmol} / \mathrm{l}$ and action is needed whenever the sodium level is below $130 \mathrm{mmol} / \mathrm{l}$. The analysis showed that these situations are often evaluated as irrelevant since no action was initiated by the pharmacist. These alerts should therefore be prevented by establishing clear cut-off levels for the generation of an alert.

Patient's data

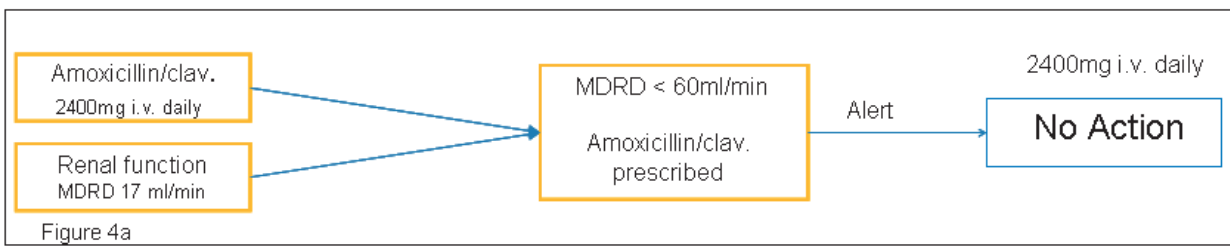

Figure 4. An example of the effect of different algorithm alert criteria. Figure 4a. Shows the algorithm alert criteria in the current system, which are limited to 'impaired renal function' and the 'prescription of a related drug'. Figure $4 \mathrm{~b}$. Shows the optimized algorithm with better specified alert criteria. 


\section{Chapter 3}

\section{CDSS optimization}

The limitations of the CDSS are shown by the daily recurrence of previously evaluated alerts. The advantage of these repeat alerts is that whenever the laboratory value changes the previously evaluated alerts, and thus the patient, can still be monitored. This is also shown by the number of actions 24 (0.9 \%) on repeat alerts (figure 2). Nevertheless, alerts that have been evaluated but are continuously reappearing without any change of one of the alert criteria (e.g. laboratory value) should be prevented. A solution to this problem would be the facility to suppress the alert until a relevant change of one of the alert criteria makes the alert reappear. For instance, the change in a laboratory value will then trigger the system to create another alert.

Further, the CDSS does not show all the information from the CPOE. The daily dosages are shown, but irregular dosage requirements such as weekly prescriptions and intermediate dosage (e.g. the stop dates) are not shown. For instance, the oral oncolytics presented in the CDSS still need to be manually checked for the stop date. Other irregular dosage requirements such as the weekly prescriptions of bisphosphonates and methotrexate are also checked manually in the CPOE. Manual checking of the CPOE can be prevented when the CDSS is programmed to show directly all relevant information.

\section{Data delivery}

When the CDSS is programmed to present all relevant information then data delivery to the CDSS should also be optimal. Delay in data delivery can lead to more unnecessary alerts and manual checking of data. Currently, the laboratory values are extracted overnight and used in the course of the next day. However, the laboratory values are routinely checked by taking blood from the patient early in the morning. These results are then not yet included in the overnight extraction causing the data to be somewhat delayed. This results leads to the necessity for manual checking of recent laboratory values and therefore some alerts are evaluated as 'no action required'. The optimal solution to this problem would be a direct link (HL7) between the laboratory system and the CDSS so that values are always up-to-date. This direct link would also aid the pharmacist with the handling of relevant alerts since the most recent laboratory value would therefore be available to be checked before the physician is contacted.

\section{Strengths and weaknesses}

The analysis of the alerts was not validated; the researcher merely consulted a trained pharmacist in evaluating the alerts when in doubt. Furthermore, the clinical relevancy of the alerts was determined by checking whether or not the pharmacist contacted the physician for each alert. Although the guidelines based clinical rules were developed with and accepted by the physicians within the Zuyderland Medical Centre the determi- 
nation of the clinical relevance of the alerts is limited to whether the physician was consulted rather than the outcome of the consult.

Some clinical rules appear not to be relevant since the NAE is $0 \%$. Nevertheless, these clinical rules are potentially important as the specific medication can always be prescribed. When the algorithm alert criteria are specified clinical rules with limited to zero efficiency will not be reported but will be enable the completion of medication surveillance. Essentially an alert efficiency of $100 \%$ would represent the optimal clinical rule, when only considering the alerts triggered (true and false positive). However, the false and true negatives were not included in this analysis. It is believed that an overall efficiency of $80 \%$ is achievable in the analyzed clinical rules. Higher efficiency will most probably result in more false negative alerts since the algorithm becomes even more specific. When considering the implementation or development of very complex clinical rules, such as the prediction of a delirium, this would necessarily have a lower sensitivity and specificity compared to clinical rules that involve the implementation of a straightforward guideline such as drug dosage in renal impairment (22).

\section{Future developments}

The categories that influence the efficiency of clinical rules; algorithm alert criteria, CDSS construction, and data delivery have also been reported in other studies. Ash et al. described the importance of different categories in the development and implementation of a CDSS. Two of the described themes: 'content component: knowledge management' and a 'technology component: data as a foundation' are also applicable to the system described in this work (23). The content theme: 'knowledge management' describes the planning and sorting of all collected information, whereas the technology theme: 'data as a foundation' describes the problems which can arise when collecting data from laboratories or making data electronically available. More specifically, Beccaro et al. also showed the positive effect of specifying the algorithm alert criteria. However, the alert criteria used were in relation to regular reference drug dosages or dosages in relation to age, weight or route of administration. In addition, drug-drug interactions were merely downgraded based on the severity and probability of an adverse reaction. However, this approach as a configuration will possibly lead to an increase in the number of false negative alerts (24). A review by Horsky et al. also describes the two above mentioned categories to reduce excessive alerting by; not triggering the alerts when certain conditions apply and by suppressing redundant alerts (25). Yet, another study which also integrated laboratory values and other patient specific parameters also suggested similar improvements as in this study such as determining clear cut-off points for triggering an alert and the suppression of duplicate alerts. These improvements can be also be related to the described categories 'algorithm alert criteria' and 'CDSS construction'. Moreover, it became clear that problems can be site specific, since laboratory data are already integrated via a HL7 data link in this study (26). 


\section{Chapter 3}

Work has now begun to develop a new CDSS rather than trying to improve the current system. During the development, all of the described recommendations of this study will be introduced. The development of clinical rules with a better efficiency will require great effort since many alert criteria need to be added or adjusted. However, not all the problems of the CDSS are efficiency-related. The new CDSS also needs to simplify the implementation of clinical rules based on guidelines and indications such as clinical rule alerting with regard to the prescribing of gastrointestinal prophylaxis. The implementation of a new CDSS with integrated guidelines may be an important tool in order to improve the quality of care and support prescribing according to the most recent guidelines $(9,27)$.

The CDSS should be validated, easy to implement, use recognised standards for sharing decision support content, easy to use in daily practice and provide information on previous alerts and actions. The advantage of using recognised standards for sharing decision support content is to enable the implementation of the CDSS in any setting. Similarly to the integration of the G-standard into the CPOE, the clinical rules from the newly developed CDSS will be integrated into CPOE. In order to avoid alert fatigue the CDSS will need be optimized before integration into the CPOE. Taking into account the patients' medical condition the physician will then be alerted directly at the point of prescribing. Successful integration would result in an efficient system and personalized medicine with minimal routine effort.

\section{CONCLUSIONS}

This study shows a number of points for improvement for the standalone CDSS currently in use in the Zuyderland Medical Centre. The average efficiency of the CDSS is low and only a few clinical rules have an efficiency greater than $10 \%$. The current system needs to be improved to reduce alert fatigue. Three categories of importance have been defined in order to improve the efficiency of the analyzed CDSS; algorithm alert criteria, CDSS optimization and data delivery.

The algorithms should take into account more variables such as the limits of dosage frequencies. Optimizing the CDSS can also diminish the number of alerts by suppressing already evaluated alerts. Similarly to irrelevant clinical alerts, the reappearance of already evaluated alerts is not only time consuming but also results in alert fatigue, and hence it is important that this issue is addressed. Data delivery can also increase the efficiency by having up-to-date data extraction or a direct link between the CDSS and hospital systems. 


\section{REFERENCES}

1. Dean B, Barber N, Schachter M. What is a prescribing error? Qual Health Care. 2000;9(4):232-7.

2. Roughead EE, Gilbert AL, Primrose JG, Sansom LN. Drug-related hospital admissions: a review of Australian studies published 1988-1996. Medical J Austr. 1998;168(8):405-8.

3. Leendertse AJ, Egberts AC, Stoker LJ, van den Bemt PM, Group HS. Frequency of and risk factors for preventable medication-related hospital admissions in the Netherlands. Arch Intern Med. 2008;168(17): 1890-6.

4. Royal Dutch Pharmacists Association (KNMP). Handboek medicatiebewaking [Internet]. 2013. [cited on 20 Dec. 2014] Available from: https://www.knmp.nl/patientenzorg/medicatiebewaking/handboekmedicatiebewaking-2013

5. Ammenwerth E, Schnell-Inderst P, Machan C, Siebert U. The effect of electronic prescribing on medication errors and adverse drug events: a systematic review. J Am Med Inform Assoc. 2008;15(5):585-600.

6. Eslami S, de Keizer NF, Abu-Hanna A. The impact of computerized physician medication order entry in hospitalized patients--a systematic review. Int J Med Inform. 2008;77(6):365-76.

7. Khajouei R, Wierenga PC, Hasman A, Jaspers MW. Clinicians satisfaction with CPOE ease of use and effect on clinicians' workflow, efficiency and medication safety. Int J Med Inform. 2011;80(5):297-309.

8. Rommers MK, Teepe-Twiss IM, Guchelaar HJ. A computerized adverse drug event alerting system using clinical rules: a retrospective and prospective comparison with conventional medication surveillance in the Netherlands. Drug Saf. 2011;34(3):233-42.

9. Jaspers MW, Smeulers M, Vermeulen H, Peute LW. Effects of clinical decision-support systems on practitioner performance and patient outcomes: a synthesis of high-quality systematic review findings. J Am Med Inform Assoc. 2011;18(3):327-34.

10. Pearson SA, Moxey A, Robertson J, Hains I, Williamson M, Reeve J et al. Do computerised clinical decision support systems for prescribing change practice? A systematic review of the literature (1990-2007). BMC Health Serv Res. 2009;9:154.

11. KNMG. Richtlijn elektronisch voorschrijven [Internet]. 2013. [ cited on 20 May 2014] Available from: http://knmg.artsennet.nl/Publicaties/KNMGpublicatie/136411/Richtlijn-elektronisch-voorschrijven2013.htm.

12. Kane-Gill SL, Visweswaran S, Saul MI, Wong AK, Penrod LE, Handler SM. Computerized detection of adverse drug reactions in the medical intensive care unit. Int J Med Inform. 2011;80(8):570-8.

13. Tawadrous D, Shariff SZ, Haynes RB, lansavichus AV, Jain AK, Garg AX. Use of clinical decision support systems for kidney-related drug prescribing: a systematic review. Am J Kidney Dis. 2011;58(6):903-14.

14. Rind DM, Safran C, Phillips RS, Wang Q, Calkins DR, Delbanco TL et al. Effect of computer-based alerts on the treatment and outcomes of hospitalized patients. Arch Intern Med. 1994;154(13):1511-7.

15. Gaweda AE, Jacobs AA, Aronoff GR, Brier ME. Model predictive control of erythropoietin administration in the anemia of ESRD. Am J Kidney Dis. 2008;51(1):71-9.

16. van der Sijs H, Aarts J, Vulto A, Berg M. Overriding of drug safety alerts in computerized physician order entry. J Am Med Inform Assoc. 2006;13(2):138-47.

17. van Roon EN, Flikweert S, le Comte M, Langendijk PN, Kwee-Zuiderwijk WJ, Smits $P$ et al. Clinical relevance of drug-drug interactions : a structured assessment procedure. Drug Saf. 2005;28(12):1131-9.

18. Eppenga WL, Derijks HJ, Conemans JM, Hermens WA, Wensing M, De Smet PA. Comparison of a basic and an advanced pharmacotherapy-related clinical decision support system in a hospital care setting in the Netherlands. J Am Med Inform Assoc. 2012;19(1):66-71.

19. Gisbert JP, Gomollon F. Thiopurine-induced myelotoxicity in patients with inflammatory bowel disease: a review. Am J Gastroenterol. 2008;103(7):1783-800.

20. Informatorium Medicamentorum. Royal Dutch Pharmacists Association (KNMP).

21. Goldsmith P, Roach A. Methods to enhance the safety of methotrexate prescribing. J Clin Pharm Ther. 2007;32(4):327-31. 


\section{Chapter 3}

22. van den Boogaard M, Pickkers P, Slooter AJ, Kuiper MA, Spronk PE, van der Voort PH et al. Development and validation of PRE-DELIRIC (PREdiction of DELIRium in ICu patients) delirium prediction model for intensive care patients: observational multicentre study. BMJ. 2012;344:e420.

23. Ash JS, Sittig DF, Guappone KP, Dykstra RH, Richardson J, Wright A et al. Recommended practices for computerized clinical decision support and knowledge management in community settings: a qualitative study. BMC Med Inform Decis Mak. 2012;12:6.

24. Beccaro MA, Villanueva R, Knudson KM, Harvey EM, Langle JM, Paul W. Decision Support Alerts for Medication Ordering in a Computerized Provider Order Entry (CPOE) System: A systematic approach to decrease alerts. Appl Clinical Inform. 2010;1(3):346-62.

25. Horsky J, Phansalkar S, Desai A, Bell D, Middleton B. Design of decision support interventions for medication prescribing. Int J Med Inform. 2013;82(6):492-503.

26. Reichley RM, Seaton TL, Resetar E, Micek ST, Scott KL, Fraser VJ et al. Implementing a commercial rule base as a medication order safety net. J Am Med Inform Assoc. 2005;12(4):383-9.

27. Fossum M, Alexander GL, Ehnfors M, Ehrenberg A. Effects of a computerized decision support system on pressure ulcers and malnutrition in nursing homes for the elderly. Int J Med Inform. 2011;80(9):607-17. 


\section{Chapter}

\section{Development of a computer system to support medication reviews in nursing homes}

This chapter is based on:

de Wit HAJM, Mestres Gonzalvo C, Hurkens KPGM, Mulder WJ, Janknegt R, Verhey FR, Schols JMGA, van der Kuy PHM.

Development of a computer system to support medication reviews in nursing homes. Int J Clin Pharm. 2013;35(5):668-72. 


\section{ABSTRACT}

The frail elderly populations of nursing homes frequently use drugs and suffer from considerable comorbidities. Medication reviews are intended to support evidence based prescribing and optimise therapy. However, literature is still ambiguous regarding the optimal method and the effects of medication reviews. Innovative computerised systems may support the medication reviews in the future. We are developing a clinical decision support system (CDSS) that, independently of the prescribing software, continuously monitors all prescribed drugs while taking into account co-medication, laboratory-data and co-morbidities. The CDSS will be developed in five phases: 1) development of the computerised system, 2) development of the clinical rules, 3) validation of the CDSS, 4) randomised controlled trial, and 5) feasibility for implementation in different nursing homes. The clinical decision support system aims at supporting the traditional medication review. 


\section{INTRODUCTION}

In 200911.8 million of the Dutch population (71.6\%) used prescription medication. A considerable number, 2.3 million (14.3\%), using prescribed medication were 65 years of age or older. In 2009, nursing homes and homes for the elderly provided care to approximately 155.000 patients daily (1). The populations of nursing homes are often frail given their inability to withstand negative events. Frail elderly is defined as the combination of biological, functional, cognitive, and clinical aspects of the elderly (2).

Polypharmacy, the use of many different drug combinations, increases the risk of adverse effects, often causes problems with patient compliance, increases the likelihood of inappropriate prescription, and simultaneously may cause suboptimal treatment because the probability of underprescription paradoxically increases with the number of drugs used $(3,4)$. A prevalence of $12 \%$ and $40 \%$ was found for potentially inappropriate medication in community-dwelling elderly and nursing home residents respectively (5). Polypharmacy also has other consequences such as increased drug interactions, adverse drug reactions, and non-adherence. Decreased physical functioning and ability to carry out instrumental activities of daily living, poorer nutritional status, increased risk of geriatric syndromes, and more falls are also associated with polypharmacy (6). Evidence-based guidelines for geriatrics and nursing homes are limited since older patients and patients with comorbidities are generally excluded from clinical trials (7). Medication prescription should be based on the physical condition of the patient with special interest in the hepatic and renal function. Regular revision of the medication should be considered, especially in elderly patients.

\section{Medication reviews}

A medication review is defined as a structured evaluation of the patient's medicines, aimed at reaching agreement with the patient, optimising the impact of medicines and minimising the number of medication-related problems while considering medical history and laboratory values (8). The Dutch Healthcare Inspectorate expects all residents of nursing homes (twice a year) and homes for the elderly (once a year) to receive a medication review by a physician and a pharmacist. In this medication review the information given by the nursing staff and the patient him/herself should be taken into account (9).

Several methods have been described to optimise prescribing and to support medication reviews $(10,11)$. Alldred et al. showed in a review for optimising prescribing in older people in care homes that there is no evidence for an effect on primary outcomes such as adverse drug events, hospital admission, and mortality. The interventions did lead to the improvement of medication-related problems (12). This shows that the mainly pharmacist initiated medication reviews create a multidisciplinary interaction between physicians, nurses, and pharmacist which optimises prescriptions and therapy. 


\section{Chapter 4}

It seems reasonable to assume that manual methods used to support medication reviews are as good as the professional using it. Outcomes will depend on variables such as knowledge of guidelines or perhaps the focus of the healthcare professionals. A study by Thier et al. has shown that the adherence to guidelines in clinical practice is low for many chronic diseases (13). Also, the current methods to support medication reviewing are time-consuming and have temporary effects since they are used manually and not continuously. Therefore, a computerised system might support a continuous, complete and reproducible medication review. Clinical rules are able to combine the pharmacy, clinical as well as laboratory data according to specifically developed algorithms (see figure 1). A computer system that can execute the algorithm based clinical rules is also referred to as a clinical decision support system (CDSS) and it can provide around the clock surveillance. Studies have shown that guidelines and clinical management tables integrated in a CDSS help healthcare professionals to avoid errors and improve clinical practice (14).

Moreover, the benefits of using a CDSS in a hospital setting are also shown $(14,15)$. Nevertheless, the use in nursing homes is still limited (16). Alldred et al. also concluded that cluster-randomised controlled trials testing a CDSS are needed to determine important population related-outcomes (12).

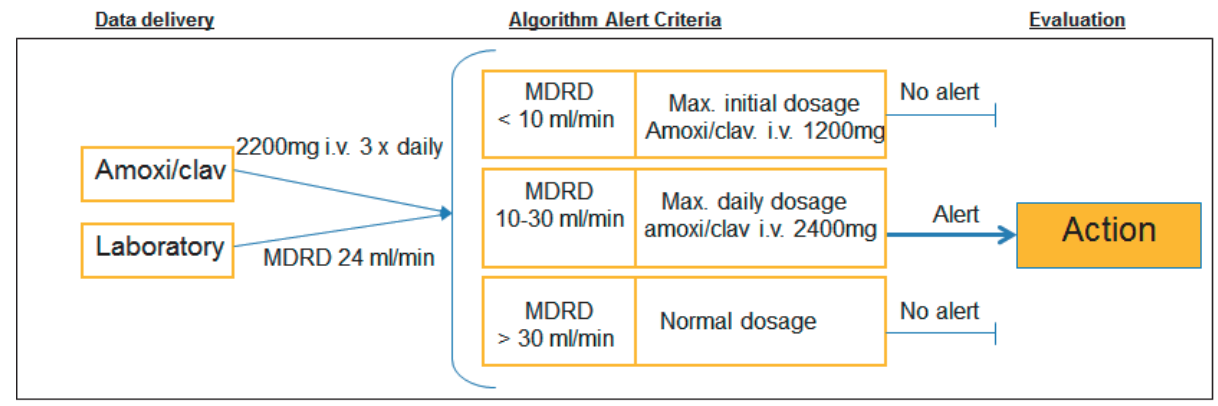

Figure 1. An example of an algorithm based clinical rule combining laboratorium and pharmacy data.

\section{New Approach}

The use of a CDSS seems to be the best direction for further optimisation and improvement of drug therapy. Currently the alerts of clinical rules used in a CDSS are strongly related to suggestions made in a medication reviews, however the alerts are underdeveloped and not yet validated for the nursing home population. It is our aim to develop a CDSS to support the traditional medication review in nursing home patients which monitors continuously the drugs used by the patient, taking into account comedication, laboratory-data and other clinical relevant data. This CDSS should be validated, easy to implement, EPS independent, easy to use in daily practice, and provide information on previous alerts and undertaken actions. Furthermore, the accessibility of 
different electronic patient's records (EPR) is pivotal for the usage of clinical rules with the primary indication for a drug as a starting point of the algorithms.

\section{Study Phases}

To achieve this, we have planned to execute the SCREEN study -Supporting Clinical Rules in the Evaluation of Elderly patients with Neuropsychiatric disorders. This study is divided in five phases (figure 2 ) and will be discussed below.

\section{Phase 1: Development of a computerised system}

The first phase is the development of a CDSS, which can use extractions from different electronic prescribing systems (EPS) and electronic patient's records (EPR). When the CDSS can use different EPS's it can be implemented in different nursing homes and probably other clinical settings such as primary care, homes for the elderly and hospital care.

\section{Phase 2: Development of the clinical rules}

Clinical rules are the content of the CDSS. The clinical rules will be divided into different topics; 1) clinical rules for general medicine, 2) laboratory values, and 3) neuropsychiatric disorders and indications. Each topic will be systematically approached through an exploration of the literature, guidelines and summaries of product characteristics. A hospital pharmacist and a physician geriatrician will explore the available literature first and design clinical rules. The concept clinical rules will then be reviewed by an expert panel consisting of hospital pharmacists, geriatricians, nursing home physicians, and physicians in neuropsychiatry and old age psychiatry.

The clinical rules for general medicine and laboratory values will consider dose adjustment for impaired renal and/or hepatic function, drugs contra-indicated for specific diseases and necessary laboratory checks to optimise pharmacotherapy such as the prescribing of an ACE-inhibitor in impaired renal failure and monitoring the potassium levels. The development of clinical rules based on guidelines will depend on the extraction of the EPR-data. For instance, a clinical rule specifying the optimal therapy after a myocardial infarction will be dependent of the known indication from the EPRextraction.

\section{Phase 3: Validation of the CDSS}

A high efficiency of the alerts in the CDSS is of great importance because many false positive alerts will lead to alert fatigue. Other studies involving prescribing software have shown the relation between the non-adherence of prescribing physicians and the appearance of too many non-relevant warnings $(17,18)$. To ensure a high efficiency the CDSS with the clinical rules will be validated via three methods. First the CDSS will be validated technically to ensure a correct link between the CDSS and the used clinical 


\section{Chapter 4}

information. This technical validation will be done by creating a fictive patient group which will include patients with every possible parameter variation in the clinical rules. By doing this, false negative alerts will be prevented. Secondly, when possible the clinical rules will be validated retrospectively by using a patient database.

Thirdly, a prospective validation will be performed to measure the effect of the clinical rule in daily practice. Whether a certain clinical rule is effective or not, can also be measured by evaluating the responses of the physicians. Alerts from clinical rules with no actions by the physicians should be evaluated on clinical relevance or specificity. The prospective validation will further facilitate the efficiency of the clinical rules.

Phase 4: Randomised controlled trial

A randomised controlled trial in nursing homes will be performed to show the possible benefits of the medication review performed by the CDSS in comparison with the traditional medication review. Outcomes are the total number of interventions suggested by the programme versus a medication review, quality of life-score, reported adverse effects (such as falling and delirium), and number of drug-related hospital admissions.

\section{Phase 5: Feasibility}

The final phase is to implement the system in other nursing homes with different software systems. The researchers will supervise this phase. The implementation in new nursing homes will need to overcome problems which might vary from technical problems extracting data from different EPS and ERPs to the introduction of a new system in an organisation.

\section{CONCLUSION}

The SCREEN-study aims to develop a generic applicable CDSS for nursing homes able to support medication reviews. The clinical trial will show the feasibility of the system to perform medication reviews in nursing homes. The lack of patient input is a limitation of the system should it replace the traditional medication review. However, the CDSS is expected to support the traditional medication review and it can serve as a secondgeneration medication surveillance system. In our opinion the development of a system, which is able to reduce the time-consuming process of a traditional medication review and create high-quality suggestions for therapy optimisation, will be unique. 


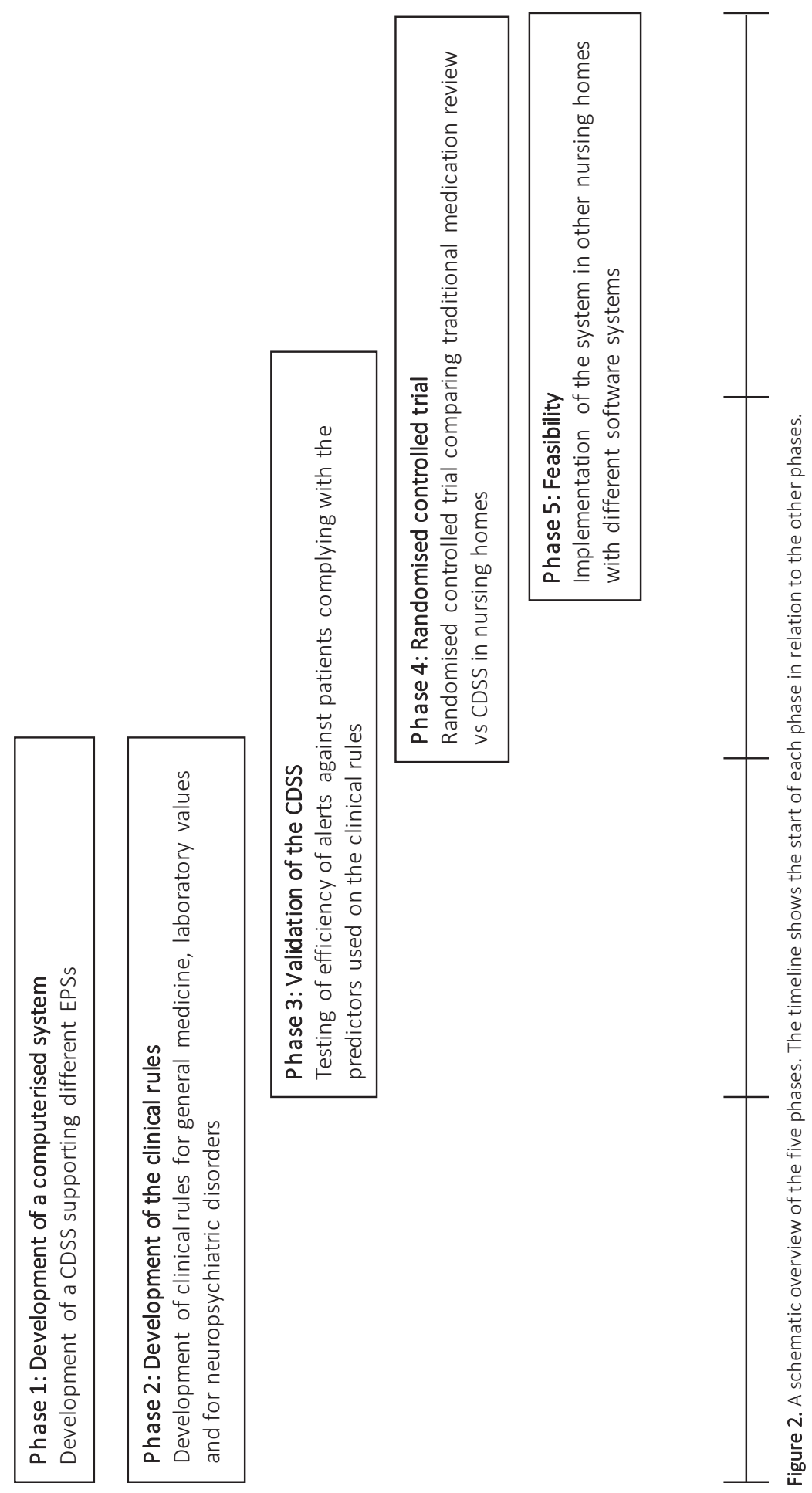




\section{REFERENCES}

1. Centraal Bureau voor de Statistiek. Open database [Internet]. [cited on 13 Feb 2012]. Available from: www.cbs.nl. Dutch.

2. Malaguarnera M, Vacante M, Frazzetto PM, Motta M. What is the frailty in elderly? Value and significance of the multidimensional assessments. Arch Gerontol Geriatr. 2013;56(1):23-6.

3. Gallagher PF, Barry PJ, Ryan C, Hartigan I, O'Mahony D. Inappropriate prescribing in an acutely ill population of elderly patients as determined by Beers' Criteria. Age Ageing. 2008;37(1):96-101.

4. Cherubini A, Corsonello A, Lattanzio F. Underprescription of beneficial medicines in older people: causes, consequences and prevention. Drugs Aging. 2012;29(6):463-75.

5. Gallagher P, Barry P, O'Mahony D. Inappropriate prescribing in the elderly. J Clin Pharm Ther. 2007;32(2):113-21

6. Shah BM, Hajjar ER. Polypharmacy, adverse drug reactions, and geriatric syndromes. Clin Geriatr Med. 2012;28(2):173-86.

7. Knottnerus A, Dinant GJ. Medicine based evidence, a prerequisite for evidence based medicine. BMJ. 1997;315(7116):1109-10.

8. Blenkinsopp A, Bond C, Raynor DK. Medication reviews. Br J Clin Pharmacol. 2012;74(4):573-80.

9. Dutch Healthcare Inspectorate. Beoordelingskader medicatieveiligheidverpleeghuizen [Internet]. [cited on 2013 June 26]. Available from: http://www.igz.nl/Images/Bijlagen\%20Beoordelingskaders\%20en \%20Risicoscore\%20bij\%20rapport\%20Medicatieveiligheid_tcm294-287277.pdf.

10. Drenth-van Maanen AC, van Marum RJ, Knol W, van der Linden CM, Jansen PA. Prescribing optimization method for improving prescribing in elderly patients receiving polypharmacy: results of application to case histories by general practitioners. Drugs Aging. 2009;26(8):687-701.

11. Gallagher PF, O'Connor MN, O'Mahony D. Prevention of potentially inappropriate prescribing for elderly patients: a randomized controlled trial using STOPP/START criteria. Clin Pharmacol Ther. 2011;89(6):845-54.

12. Alldred DP, Raynor DK, Hughes C, Barber N, Chen TF, Spoor P. Interventions to optimise prescribing for older people in care homes. Cochrane Database Syst Rev. 2013;2:CD009095.

13. Thier SL, Yu-Isenberg KS, Leas BF, Cantrell CR, DeBussey S, Goldfarb NI et al. In chronic disease, nationwide data show poor adherence by patients to medication and by physicians to guidelines. Manag Care. 2008;17(2):48-52, 5-7.

14. Jaspers MW, Smeulers M, Vermeulen H, Peute LW. Effects of clinical decision-support systems on practitioner performance and patient outcomes: a synthesis of high-quality systematic review findings. J Am Med Inform Assoc. 2011;18(3):327-34.

15. Tawadrous D, Shariff SZ, Haynes RB, lansavichus AV, Jain AK, Garg AX. Use of clinical decision support systems for kidney-related drug prescribing: a systematic review. Am J Kidney Dis. 2011;58(6):903-14.

16. Fossum M, Alexander GL, Ehnfors M, Ehrenberg A. Effects of a computerized decision support system on pressure ulcers and malnutrition in nursing homes for the elderly. Int J Med Inform. 2011;80(9):607-17.

17. Magnus D, Rodgers S, Avery AJ. GPs' views on computerized drug interaction alerts: questionnaire survey. J Clin Pharm Ther. 2002;27(5):377-82.

18. Glassman PA, Simon B, Belperio P, Lanto A. Improving recognition of drug interactions: benefits and barriers to using automated drug alerts. Med Care. 2002;40(12):1161-71. 


\section{ADDENDUM 1}

Computer system to support medication reviews: a good but not new concept. Bindoff IK, Peterson GM, Curtain C. Int J Clin Pharm. 2014;36(2):218-9 (with permission).

In their recent article, de Wit and colleagues put forward a solid argument for the use of clinical decision support systems to support medication reviews for the elderly (1). They point out that medication reviews are routinely performed, and they have positive effects in terms of reducing medication-related problems. They also rightly note that the quality of the findings and recommendations contained within these medication reviews are only going to be as good as the knowledge and skills of the professionals performing them. They then argue that a clinical decision support system using clinical rules that combine the available pharmacy, clinical and laboratory data may be able to improve the delivery of medication reviews. This we also accept.

The authors then assert that doing this would be a "new approach", and outline a simple plan for how they intend to develop this innovative system. In their conclusions, they state "in our opinion the development of a system, which is able to reduce the time-consuming process of a traditional medication review and create high-quality suggestions for therapy optimisation, will be unique". We wish to highlight that this approach is not unique.

Over the past 8 years, we have developed and evaluated systems that can provide intelligent clinical decision support for medication reviewers, and have presented the results at a range of conferences and in international publications (2-4). In these publications we described the rationale for and the implementation of an advanced system that allows expert medication reviewers to seamlessly and naturally build complex rules based on a patient's biographical details, medications, medical history, and laboratory results, while performing their routine medication review duties. The approach we used for doing this is based on recent advances in the field of knowledge acquisition (a subfield of artificial intelligence). This approach allows the expert to iteratively build up and refine a large and complex set of rules that are automatically used to identify medication-related problems. If the system ever identifies a problem that the expert disagrees with, or otherwise fails to identify a problem the expert has detected, the expert is asked to indicate what the problem should have been and why. In this way the system learns over time and the recommendations are highly individualised. Importantly, with this approach, very few false positives are identified, as the system's knowledge is gradually refined such that problems are only identified when it is definitely appropriate to do so in the particular case. Even when best practice suddenly changes, the system is easily updated to reflect the newest information (4).

With relatively little training our system was capable of identifying approximately 90 $\%$ of all medication-related problems for a patient. Importantly, the system was capable 


\section{Chapter 4}

of identifying problems that the expert had missed or incorrectly classified (errors). On average, we found that the system prevented the expert from making 2.02 errors per patient (2).

Furthermore, our approach was incorporated into a commercially available system to support medication reviewers. This product has since been used by pharmacists to perform more than 50,000 medication reviews. We have also compared the medication-related problems found by this product against original pharmacist's findings, and even against the findings found by applying the STOPP/ START guidelines (3, 5). A 12 member expert panel consisting of accredited medication review pharmacists, physicians, and pharmacologists was asked to rate the problems identified by each method in terms of their clinical relevance and importance (3). The panel members were not told which method identified which problems. We found that the problems identified by MRM were of equivalent quality in terms of both clinical relevance and importance when compared to those identified by the original pharmacist reviewer, but MRM identified somewhat more. The problems identified by applying the STOPP/ START guidelines were also clinically relevant, but were far fewer in quantity, with many important problems not identified.

Furthermore, we wish to flag a concern with the proposal of de Wit et al. They state that they will build their clinical rules through a literature search and examination of established guidelines. This approach is known within the expert and knowledge-based systems community to be unwise, as it results in the development of systems in which the rules are difficult to validate and maintain, because the rules generated using this approach are typically not sufficiently refined at inception, and because best practice is constantly shifting from year to year. This adversely affects the sustainability of these systems (6).

To overcome this problem we used knowledge acquisition principles, in this case the Multiple Classification Ripple Down Rules approach (4), to build and maintain our rule sets. We would strongly recommend de Wit et al. do the same, or their work will be quickly out of date and routinely annoying the user with incorrect findings. 


\section{REFERENCES}

1. de Wit H, Gonzalvo C, Hurkens K, Mulder W, Janknegt R, VerheyF, et al. Development of a computer system to support medication reviews in nursing homes. Int J Clin Pharm. 2013;35(5):668-72.

2. Bindoff I, Stafford A, Peterson G, Kang BH, Tenni P. The potential for intelligent decision support systems to improve the quality and consistency of medication reviews. J Clin Pharm Ther. 2012;37(4):452-8.

3. Curtain C, Bindoff I, Westbury J, Peterson G. Validation ofdecision support software for identification of drug-related problems in home medicines reviews. In: Proceedings of the 11th National Conference of Emerging Researchers in Ageing; 2012 Nov 19-Nov 20; Brisbane, Australia.

4. Bindoff I, Kang BH, Ling T, Tenni P, Peterson G. Applying MCRDR to a multidisciplinary domain. In: Proceedings of the 20th Australian Joint Conference on Artificial Intelligence; 2007; Gold Coast, Australia: Springer.

5. Gallagher P, Ryan C, Byrne S, Kennedy J, O'Mahony D. STOPP (Screening Tool of Older Person's Prescriptions) and START (Screening Tool to Alert doctors to Right Treatment). Consensus validation. Int J Clin Pharmacol Ther. 2008;46(2):72-83.

6. Bachant J, McDermott J. R1 Revisited: four years in the trenches. Readings from the Al magazine. Am Assoc Artif Intell.1984;5:177-88. 


\section{ADDENDUM 2}

A fully automated medication review?

de Wit HAJM, Mestres Gonzalvo C, Janknegt R, Schols JMGA, van der Kuy PHM. Inter J Clin Pham. 2013;36(2):220-221.

In a letter to the editor concerning the manuscript "development of a computer system to support medication reviews in nursing homes" the authors state that the proposed computer system to support medication reviews is a good concept but not new (1).

We agree the concept of a clinical decision support system (CDSS) is not new nor the idea to use these techniques to support a medication review. Furthermore, the concept of multiple classification ripple-down rules (MCRDR) methodology suggested appears to be state of the art programming. However, we believe our approach has an unique approach since the system is able to support the medication review fully electronic.

All the information used in our developed CDSS is retrieved and processed electronically. The medication, laboratory data and diagnosis are extracted from different systems and loaded into the CDSS. Bindoff et al. describe one of the software element as being a standard database "front-end" where the user can enter manually a single patient's details such medical history, medication and pathology (reed laboratory data). The average time needed for analysing each patient is 9 minutes (2). To our view this is an important limitation. Currently we automatically analyse 2000 patients daily. Entering patient's details to any system appears to be very time consuming, whereas data gathering is not even included. On the other hand our system currently does not incorporate adverse drug events. The adjustment of medication based on patient feedback is currently not possible, because adverse events are not electronically documented.

Furthermore, the author suggests the development of evidence-based clinical rules is unwise because of difficulties in validation and maintenance. We believe that "evidence-based medicine" is the best way forward. The opinion of a single pharmacist or physician to create a clinical rule can be helpful, but is not necessarily correct and can perhaps result in negative effects. Therefore, we have chosen to maintain and develop our clinical rules through the advice of an expert panel provided by feedback from physicians, pharmacist, new guidelines, and literature.

This results in a much more specific system. As described in our manuscript, the developed system only alerts when the dosage of a specific drug should be adjusted based on the kidney function. This is done by adding cut-off points for dosages for the kidney function for all relevant drugs. The alerts therefore have a maximum positive predictive value. In contrast, the demo-system shown on www.medscope.com.au merely alerts that drugs should be checked manually for dosage since the manually entered kidney function is decreased.

In addition, the described highly individualized method is a solution when pharmacists use the system as a standalone. However, in our opinion pharmacists should not 
influence the alerts of other pharmacists unless evidence-based or discussed by an expert panel. For instance we are often debating the relevant cut-off point of a 'hyperkalemia' clinical rule. The clinical relevance of hyperkalemia should be interpreted differently for a patient from a general practitioner than from a nephrologist. This can vary greatly from $>4.5 \mathrm{mmol} / \mathrm{l}$ to $>5.5 \mathrm{mmol} / /$ respectively. Designing a good clinical rule with an optimal cut-off point is difficult and should be differentiated for the patient population and not the pharmacist. Also, we believe some clinical rules cannot be developed by artificial intelligence (yet). We are developing clinical rules that will be able to predict a delirium and falls by weighing many different risk factors.

We believe our system is unique since we are developing a fully electronic engine without manually entering patients' details. In addition, we are developing 'evidencebased' clinical rules which will be implemented in a hospital setting and in nursing homes. Nevertheless, we are interested in the approach of "knowledge acquisition" and perhaps exchanging knowledge can support this field of research even further. 
Chapter 4

\section{REFERENCES}

1. de Wit HA, Mestres Gonzalvo C, Hurkens KP, Mulder WJ, Janknegt R, Verhey FR et al. Development of a computer system to support medication reviews in nursing homes. Int J Clin Pharm. 2013;35(5):668-72.

2. Bindoff IK, Tenni PC, Peterson GM, Kang BH, Jackson SL. Development of an intelligent decision support system for medication review. J Clin Pharm Ther. 2007;32(1):81-8. 


\section{Chapter}

\section{The support of a medication review in hospitalised patients by a clinical decision support system}

This chapter is based on:

de Wit HAJM, Hurkens KPGM, Mestres Gonzalvo C, Smid M, Sipers W, Winkens B, Mulder WJ, Janknegt R, Verhey FR, van der Kuy PHM, Schols JMGA.

The support of a medication review in hospitalised patients by a clinical decision support system. Springerplus. 2016;5(1):871. 


\section{Chapter 5}

\section{ABSTRACT}

\section{Introduction}

An advanced clinical decision support system (CDSS) developed in Zuyderland Medical Centre consists of algorithms that alert against the inappropriate combination of medication and laboratory values, lack of guideline implementation, and gives suggestions to prevent adverse drug events as a result of drug-disease interactions. The first aim of this study is to estimate the added value of the CDSS in the performance of medication reviews in hospitalised elderly. Second, to identify the limitations of the CDSS by analysing the drug-related problems (DRPs) generated.

\section{Methods}

Medication reviews were performed in patients admitted to the geriatric ward of the Zuyderland medical centre. Additionally, electronically available patient information was introduced into a CDSS. The DRP notifications generated by the CDSS were compared with those found in the medication review. The DRP notifications were analysed to learn how to improve the CDSS.

\section{Results}

A total of 223 DRP strategies were identified during the medication reviews. The CDSS generated 70 clinically relevant DRP notifications. Of these DRP notifications, 63\% (44) were also found during the medication reviews. The CDSS generated $10 \%$ (26) new DRP notifications and conveyed 28\% (70) of all 249 clinically relevant DRPs that were found. Classification of the CDSS generated DRP notifications related to 'medication error type' revealed that 'contraindications/interactions/side effects' and 'indication without medication' were the main categories not identified during the manual medication review. The error types 'medication without indication', 'double medication', and 'wrong medication' were mostly not identified by the CDSS.

\section{Conclusions}

The CDSS used in this study is not yet sufficiently advanced to replace the manual medication review, though it does add value to the manual medication review. The strengths and weaknesses of the current CDSS can be determined according to the medication error types. 


\section{INTRODUCTION}

Frailty in the aging patient is a state of vulnerability in which health status can suddenly decrease as a result of relatively small health events (1). Ultimately frailty may lead to considerable disability. Frail elderly often have multiple chronic conditions which are associated with the use of many drugs. Polypharmacy is often defined as the use of more than five drugs per patient. This is an arbitrarily chosen cut-off point and varies between studies. Alternatively, polypharmacy can be defined as the use of a higher number of drugs than clinically indicated $(2,3)$. Both polypharmacy and frailty are independently associated with morbidity intensity suggesting a direct effect of polypharmacy on a patient's frailty status. The influence of polypharmacy on frailty might be explained by decreased compliance, more adverse drug reactions (ADR), and drug interactions. Excessive polypharmacy, which is the use of more than ten drugs, is an independent risk factor for mortality (4).

A drug-related problem (DRP) is "an event or a circumstance involving drug therapy that actually or potentially interferes with health care outcome" (5). DRPs are associated with (frail) elderly as a consequence of polypharmacy, complex dosing regimens as well as alterations in pharmacokinetics and pharmacodynamics. DRPs are also associated with cognitive and functional status (5).

Leendertse et al. showed that polypharmacy is an independent risk factor for hospitalisation. Of the unplanned hospital admissions 5.6\% were medication-related. Of these almost half were probably preventable (6). Paradoxically, polypharmacy has been shown to increase during hospitalisation of elderly (7).

\section{Medication review}

A medication review is defined as a structured evaluation of a patient's medication by a physician and a pharmacist, taking into account medical history and laboratory values. The medication review aims to reach agreement about drug therapy in order to optimise the impact of medication while minimising the number of medication-related problems. When the patient's input into the medication review is included with all available patient's information, the review is defined as a clinical medication review. Without the patient's input, the review is defined as a treatment review. This is often the case in hospitalised patients $(8,9)$. It has been suggested that there should be an intermediate definition for hospitalised patients, since patients are followed up daily by nursing staff and physicians, while recognising that dementing or very ill patients cannot give comments on their medication (9). In a systematic review by Christensen et al. no conclusive benefits on future hospitalisations or mortality in hospitalised patients are shown when performing medication reviews. However, performing medication reviews has shown to reduce the number of emergency department contacts (10). Furthermore, it has been shown that regular revision of medication reduces DRPs and improves 


\section{Chapter 5}

medication appropriateness (11). The inconsistency of proven benefits of medication reviews might be caused by implementation problems. These problems involve the time efficiency of performing medication reviews as well as the structure and information used in a medication review. The output consistency of the health care professionals performing the medication reviews can also be a factor (12-14). These problems might be (partially) resolved by automation of the medication review (15).

\section{Clinical decision support systems (CDSS)}

The Dutch Healthcare Inspectorate (IGZ) requires physicians to prescribe exclusively using computerised physician order entry (CPOE) systems with an integrated drug safety alert system.

In the Netherlands, a nationwide drug database is maintained by the Royal Dutch Pharmacists Association (KNMP) that generates drug safety alerts to ensure medication surveillance which include dosage appropriateness, double medication, drug-drug interactions, and drug contraindications (16). The pharmacist responsible for the CPOE can regulate the drug safety alerts to a degree (17). These alerts result from relatively uncomplicated algorithms that form a basic clinical decision support system (CDSS) (18). The use of a CDSS with advanced algorithms that combine medication and laboratory values is becoming common practice in the Netherlands, and the use of advanced algorithms that incorporate guidelines and drug-disease interactions that initiate treatment to prevent adverse drug events is up-coming (19-21). Unlike the basic algorithms that are routinely integrated into CPOEs, the advanced algorithms are mostly not integrated into a CPOE. Integration of a CDSS into a CPOE results in the presentation of alerts when a physician is prescribing medication. In this way the physician can adjust the prescription during the prescription process if necessary, instead of adjusting the prescription afterwards, such as when it is initiated by an alert from a standalone $\operatorname{CDSS}(22,17,23)$. Patient outcome benefits of using advanced CDSSs remain limited, but several studies do report positive results related to prevention of adverse drug events (19, 23-25). Bright et al. have shown in a systematic review that CDSSs do influence 'health care process measures' by improving the initiated treatment or preventive care in terms of: pharmacotherapy, laboratory test ordering, and chronic disease management (24).

In a recent study, the use of an advanced CDSS to support medication reviews in older hospitalised patients has shown to improve the appropriateness and accuracy of medication regimens (20).

The department of clinical pharmacy and toxicology of the Zuyderland medical centre has developed an advanced CDSS based on our experience with a simpler access based CDSS (22). The currently developed advanced CDSS consists of algorithms that alert against the inappropriate combination of medication and laboratory values, for lack of guideline implementation, and gives suggestions to prevent adverse drug events as a result of drug-disease interactions. The development phases of the CDSS have been 
described in more detail previously (15). The content development team consisted of several hospital pharmacists and internists, a neuropsychiatrist, and a nursing home physician. In the Netherlands, nursing home medicine is an officially recognised medical discipline for physicians attending nursing homes (26). The content was based on national guidelines, protocols and relevant studies. The CDSS consists of 469 clinical rules aiming to have a high sensitivity and specificity. This standalone CDSS has been developed to support medication reviews in synergy with the already integrated CPOE drug safety alerts that consider dosage appropriateness, double medication, drug-drug interactions, and drug contraindications.

The aim of this study was to estimate the value of this CDSS regarding the performance of medication reviews in hospitalised elderly by comparing DRPs taken from a multidisciplinary treatment review to the DRP notifications prompted by the CDSS. Furthermore, we aimed to identify the limitations of the current CDSS by analysing the CDSS generated DRP notifications.

\section{METHODS}

From November 2012 to December 2013 medication reviews were performed in clinical patients admitted to the geriatric ward of the Zuyderland medical centre, located in Sittard-Geleen. The medication reviews were performed during the weekly 'gerontopharmacology meeting'. During every gerontopharmacology meeting one patient was discussed for 0.5 to 1 hour. The patients were selected by the geriatrician and communicated to all the participants before the meeting. At least one geriatrician (leading practitioner and chairman of the meeting), one resident and one hospital pharmacist (in training) attended the meeting. In most cases, more clinicians attended, including geriatricians, residents, nurse practitioners and medical students. The meeting was also intended to provide educational value. The leading practitioner and chairman of the meeting was either a geriatrician or an internist, with a subspecialty in old age medicine. Both are geriatric experts, and have expertise in medical education. The attending hospital pharmacist had extensive experience in performing structured medication reviews, as necessary for this study. Apart from these experts, up to 5 postgraduate physician residents and 5 medical students attended the meeting.

The structure of the review was based on the method used in the PHARM-study and was followed during the medication reviews (27). This structure was: 1) Matching the prescribed medication with the known indications, 2) Matching indications with prescribed medications, 3) Relating the laboratory values to the prescribed medication, 4) A general discussion whether there were other suggestions based on the patients' or nurses input. Although the pharmacist did not perform patients' interviews, the geriatrician and residents had daily contact with the reviewed patients, and any relevant information was then presented in the meeting. The indirect input of patients' com- 
ments causes these medication reviews to supersede the requirements of a standard "treatment review", but does not meet the requirements of a "clinical medication review". For this study, we will continue to address them as medication reviews.

The hospital pharmacist prepared the medication review by documenting the DRP suggestions resulting from the manual check of medications, laboratory values, and other relevant clinical patient information. These suggestions were discussed during the medication review by the attendants. If they were accepted by the attendants as a DRP, it was included as a strategy to improve the patient's therapy. The accepted 'DRP strategies' were documented by the hospital pharmacist. A week after the medication review the hospital pharmacist documented which DRPs had indeed been executed. In this study, we will discuss the DRPs using the following terms: "DRP suggestions" (when DRPs were identified during the pharmacist's preparation), "DRP remarks" (newly introduced DRPs during the medication review), "DRP strategies" (when DRPs were accepted as therapy strategies for the patient during the medication review), "DRP notifications" (the DRPs found by the CDSS), and "DRPs executed" (the carried out therapy strategies based on the DRP strategies).

In addition, prior to the gerontopharmacology meeting the hospital pharmacist extracted all electronic available patient information: laboratory values, medication, and documented indications and contraindications. The extracted electronic patient information was introduced into the CDSS in April 2015, therefore it was not possible that the medication reviews identified DRPs were influenced by the CDSS generated DRPS. The CDSS is designed to exclusively alert against relevant DRPs (DRP notifications). This is done by including 'triggers' that allow assessing whether the predefined DRPs are relevant or non-relevant. For example, there is an algorithm alerting for the prescription of a gastrointestinal prophylaxis in a patient at risk. However, for some patients a prophylaxis will already be prescribed and this notification is therefore assessed as a non-relevant notification by the CDSS. The DRP notifications from the CDSS were independently checked for relevancy and classified for type of medication error by hospital pharmacist HW and geriatrician KM. In case of disagreement, consensus was obtained by discussing on a case by case basis.

The local Medical Research \& Ethics Committee (MREC) determined this study to be non-accessory for the Dutch Medical Research in Human Subjects Act (non-WMO).

Numerical variables were presented by mean (SD; range, i.e. minimum - maximum value) and categorical ones by number (\%). The descriptive statistics were computed using Microsoft Excel 2010.

\section{RESULTS}

We held 33 documented gerontopharmacology meetings during which 33 medication reviews were performed, all on different patients. The mean age of the reviewed pa- 
tients was 83 years (SD 8.0; range 69-97). Gender was distributed almost equally with $55 \%(n=18)$ of the patients being male. The mean amount of prescribed drugs per patient was 15.2 (SD 4.0; range 8-27 medicines).

\section{Drug-related problems}

The pharmacist who prepared the gerontopharmacology meeting had a total of 221 DRP suggestions based on the 33 medication reviews. Of these 221 DRP suggestions, 166 DRP suggestions were accepted as DRP strategies, while 55 DRP suggestions were rejected and an additional 57 DRP remarks were introduced during the meeting and accepted as DRP strategies. Thus, a total of 223 DRP strategies were accepted with a mean of 7.0 (SD 2.2; range 2-11) per patient (see figure 1).

The CDSS generated 574 DRP notifications. Of these notifications the CDSS determined 516 to be non-relevant notifications. The remaining 58 DRP notifications were determined as relevant DRP notifications by the CDSS. These 58 CDSS DRP notifications were checked by HW and KM, 51 of which were considered clinically relevant for the patient. In addition, HW and KM confirmed 19 notifications that were assessed as nonrelevant by the CDSS to be clinically relevant DRP notifications. In total 70 DRP notifications issued by the CDSS were relevant (see figure 1).

Of the confirmed relevant CDSS DRP notifications, 63\% (44) DRP notifications were also noted and accepted as DRP strategies in the medication reviews. Twenty-six DRP notifications from the CDSS had not been identified during the medication review but were assessed as clinically relevant by HW and KM. Table 1 shows the CDSS DRP notifications that went unnoticed in the medication reviews.

These 44 DRP notifications covered $20 \%$ of the DRP strategies identified in the medication review. The CDSS generated an additional 26 newly confirmed DRP strategies (see figure 1). The 223 DRP strategies from the medication reviews combined with the additional 26 confirmed relevant DRP notifications from the CDSS, add up to a total of 249 DRP strategies. The CDSS generated 28.1\% (70) of all 249 DRPs strategies of which $10 \%$ (26) were new notifications.

The 223 DRP strategies determined in the medication reviews were followed up for implementation a week after the medication review. Fifty-five percent (121) of these DRP strategies were executed a week after the medication review, which is a mean 3.8 (SD 2.3; range 0-9) DRPs executed per patient.

\section{Classification of drug-related problems}

All DRPs were categorised according to a seven-fold 'medication error type' classification (see table 2). Table 2 shows that two medication error types (a) 'indication without medication' and (b) 'medication without indication' made up more than half of the existing medication errors. The 26 relevant DRP notifications not recognised during the 


\section{Chapter 5}

medication reviews were mainly medication errors type (a) 'indication without medication' and error type (c) 'contraindications/interactions/side effects'. The error types (b) 'medication without indication', (e) 'double medication', and (f) 'wrong medication' were least likely to be identified by the CDSS.

Table 1: DRP notifications not identified in the medication review.

Potassium level - drugs inducing hypokalemia

Use of aspirin, dipyridamol, clopidogrel, prasugrel without a statin

Calcium channel blockers with chronic constipation

Atypical antipsychotics combined with oral blood glucose lowering drugs or insulin

Atypical antipsychotics combined with Alzheimer medication

Nitrate without aspirin or clopidogrel, prasugrel, ticagrelor

Metformin with unknown vitamin B12 level

Renal failure with amoxicillin (oral)

Atypical antipsychotics combined with antihypertensive medication

Nitrate without a beta blocker

Nortriptyline usage in elderly patients

Tricyclic antidepressants with constipation

Paracetamol in elderly patients in combination with risk factors

Gastric protection without prophylaxis

Gastric protection, prophylaxis dosage not high enough

Classical antipsychotics with anticholinergic effects - start alpha blockers may be related to this effect

Tricyclic antidepressants with cardiac conductive abnormalities

Use of rheumatoid arthritis drugs without a statin

Bisphosphonates, calcium and vitamin D supplementation: suggest prescribing calcium

Renal Failure with metoclopramide 
The support of a medication review in hospitalised patients by a CDSS

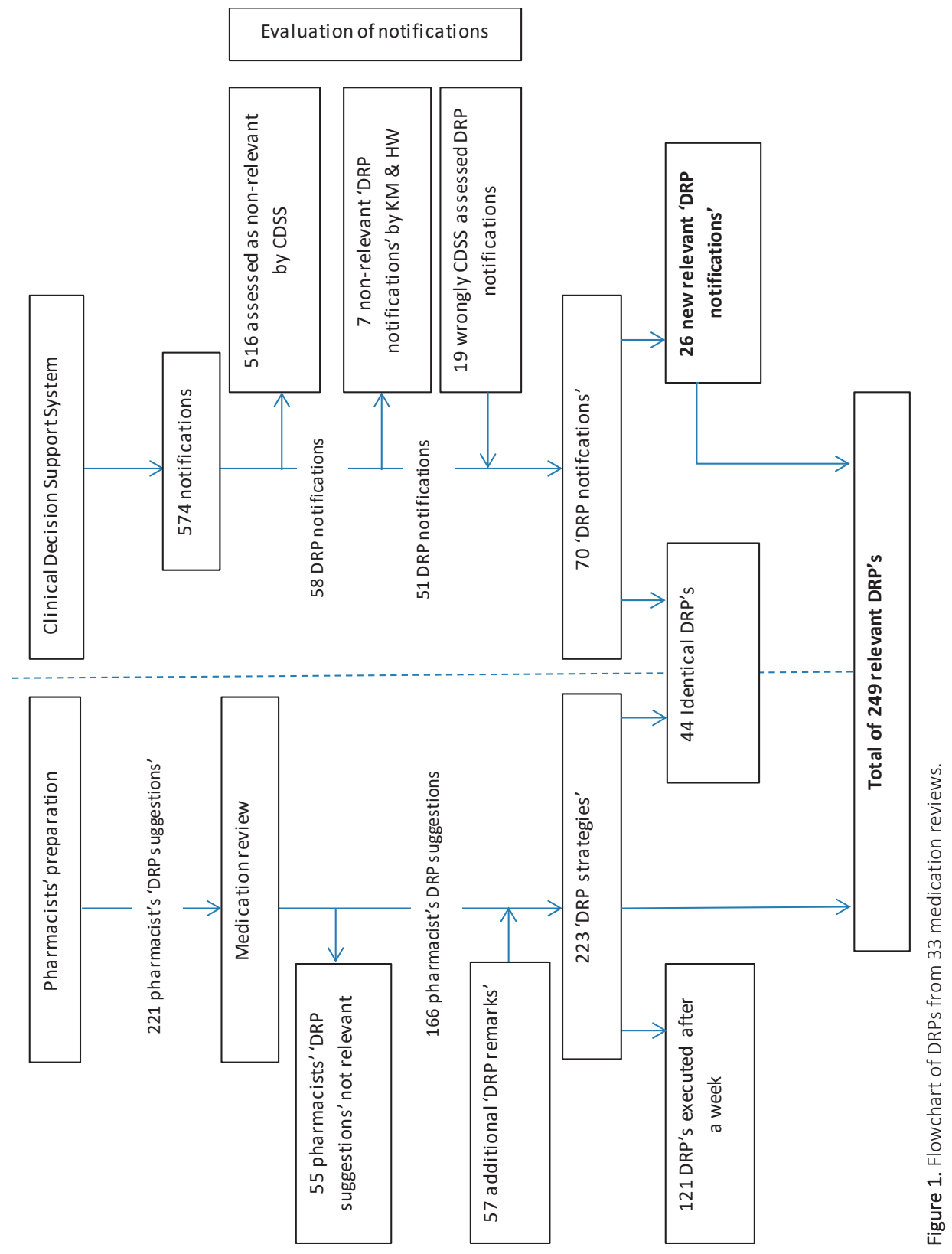


Chapter 5

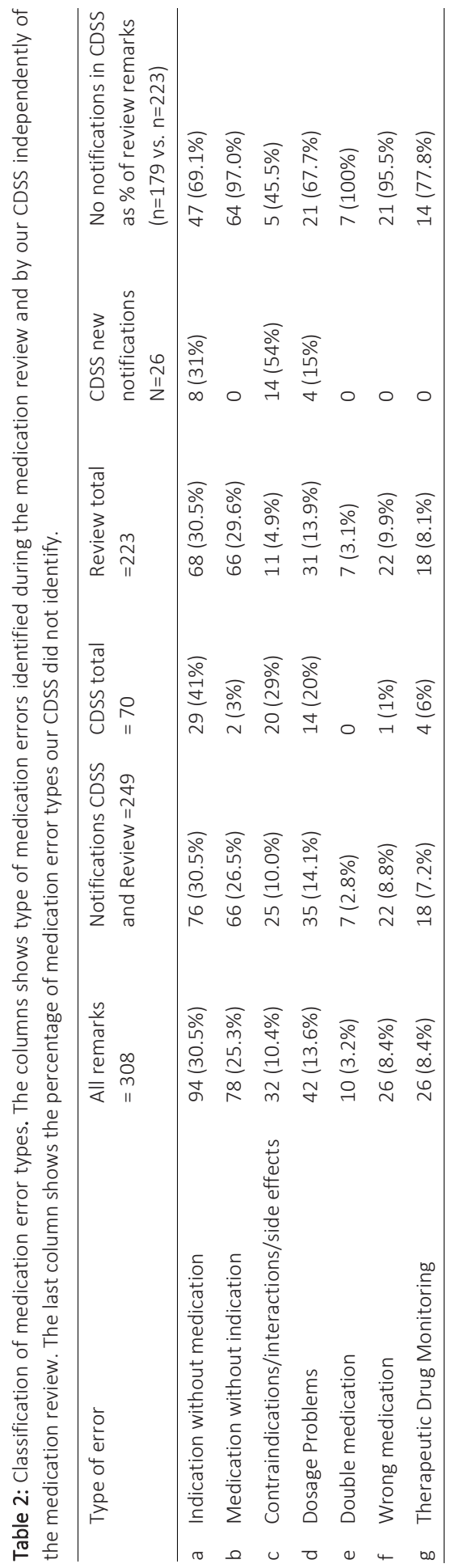




\section{CDSS efficiency}

The efficiency of the CDSS was determined by calculating the sensitivity and specificity of the CDSS using the confirmed correctness of CDSS notifications. Fifty-one of the 58 DRP notifications were found true positive, giving a sensitivity of $72.9 \%$. Of the 516 DRPs assessed as non-relevant notifications by the CDSS, 19 notifications were confirmed relevant by $\mathrm{KH}$ and $\mathrm{HW}$ giving $497 \mathrm{DRP}$ true negative notifications resulting in a specificity of $98.6 \%$. In table 3 the sensitivity and specificity are shown.

Table 3. Sensitivity $=A / A+C * 100 \% .=72.9 \%$, specificity $=D / D+B * 100=98.6 \%$.

\begin{tabular}{llcr}
\hline & \multicolumn{1}{l}{ Confirmed relevant DRPs } & \multicolumn{1}{c}{ Confirmed irrelevant DRPs } \\
\hline Relevant DRP Notifications & $51(\mathrm{~A})$ & $7(\mathrm{~B})$ & $58(\mathrm{~A}+\mathrm{B})$ \\
Non-relevant DRP Notifications & $19(\mathrm{C})$ & $497(\mathrm{D})$ & $516(\mathrm{C}+\mathrm{D})$ \\
& $70(\mathrm{~A}+\mathrm{C})$ & $504(\mathrm{~B}+\mathrm{D})$ & $\mathrm{N}=574$ \\
\hline
\end{tabular}

The confirmed clinically relevant CDSS notifications were analysed to determine the reasons why they were incorrectly assessed as relevant or irrelevant by the CDSS. In table 4, the CDSS notifications assessed as non-relevant but confirmed as relevant are shown. In table 5, the CDSS notifications assessed as relevant but confirmed as nonrelevant are shown. The actions needed to improve the sensitivity and specificity of these algorithms varies considerably and are also shown in tables 4 and 5.

\section{DISCUSSION}

This study shows that performing medication reviews in a hospitalised geriatric patient group can be of value when considering the mean of 7.0 (SD 2.2) DRP strategies identified during the gerontopharmacology meetings.

The acceptance rate of the pharmacists suggestions was $74.4 \%$ (166 out of 221 DRP suggestions) which corresponds with other described acceptance rates of DRP suggestions ranging from $39.0 \%$ to $91.6 \%$ in long-term care patients and $65 \%$ in hospitalised patients $(28,29,9)$. In our study, we also investigated to which extend the DRP strategies were executed by the physician one week after the medication reviews. This resulted in 54.3\% (122) executed DRPs after one week. Another study reports that $65 \%$ of the DRP strategies were executed (9). It should be mentioned that the patient group studied is easily susceptible to clinical changes, which might explain why physicians decided not to execute certain DRP strategies. 


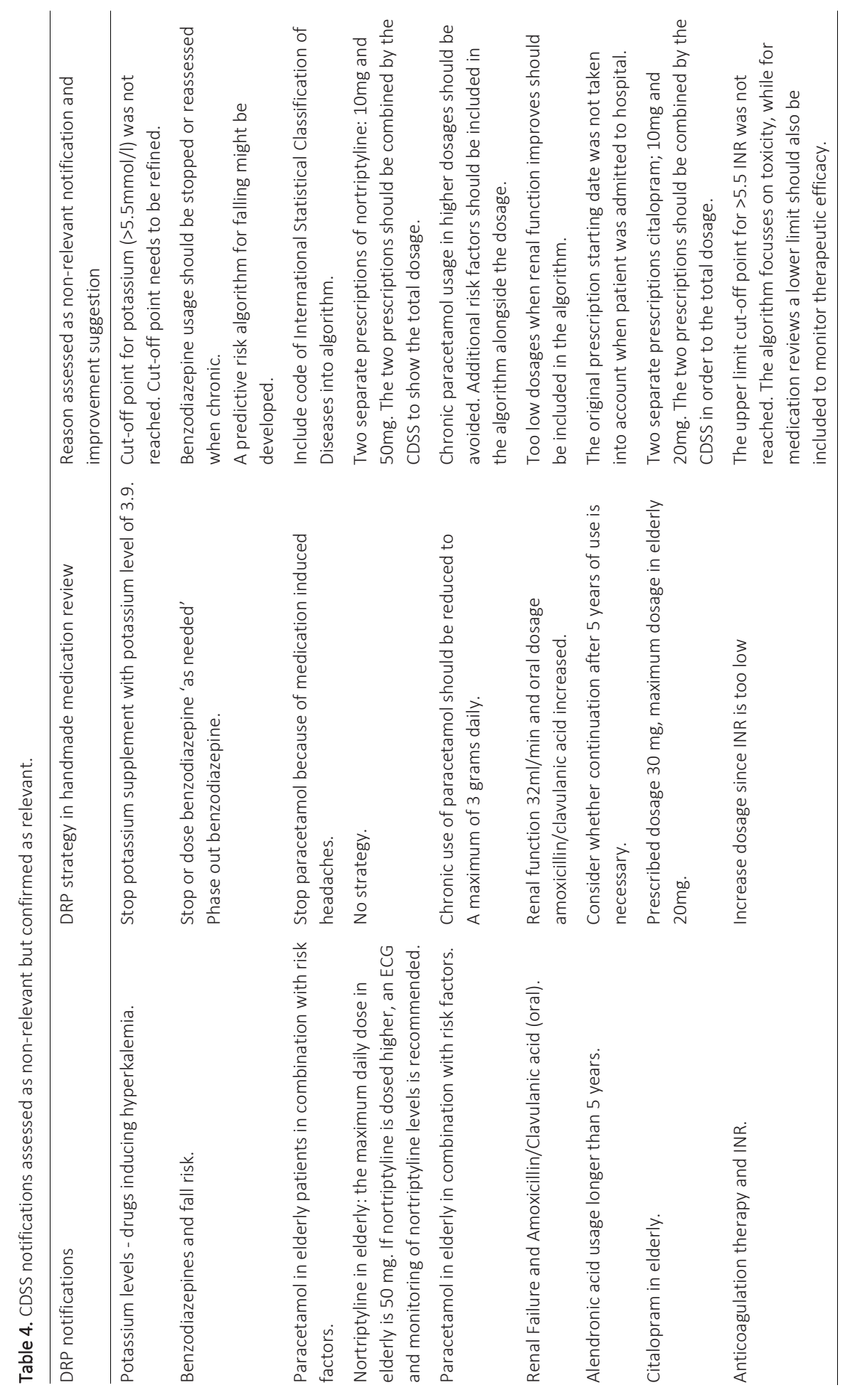


The support of a medication review in hospitalised patients by a CDSS

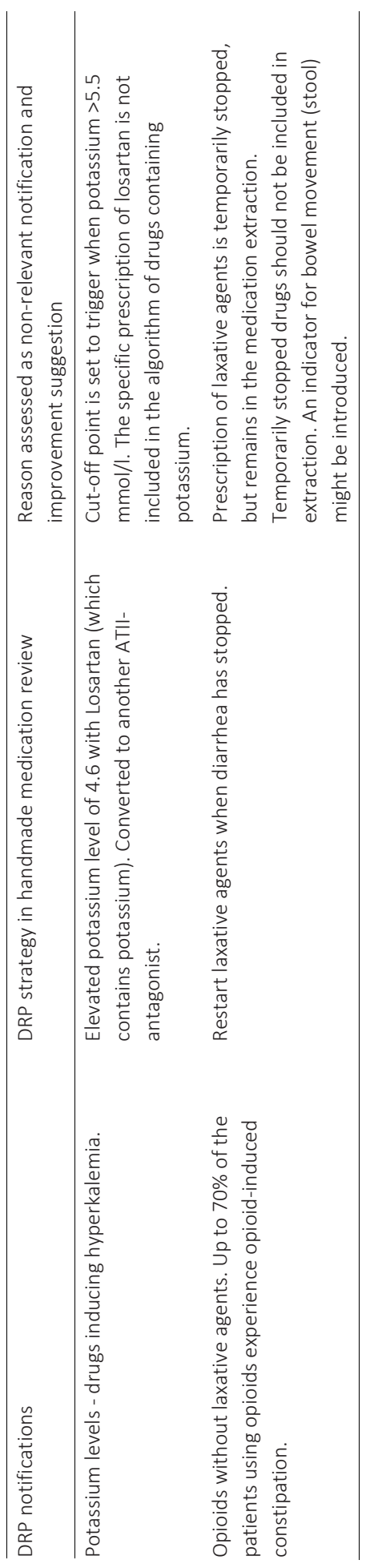




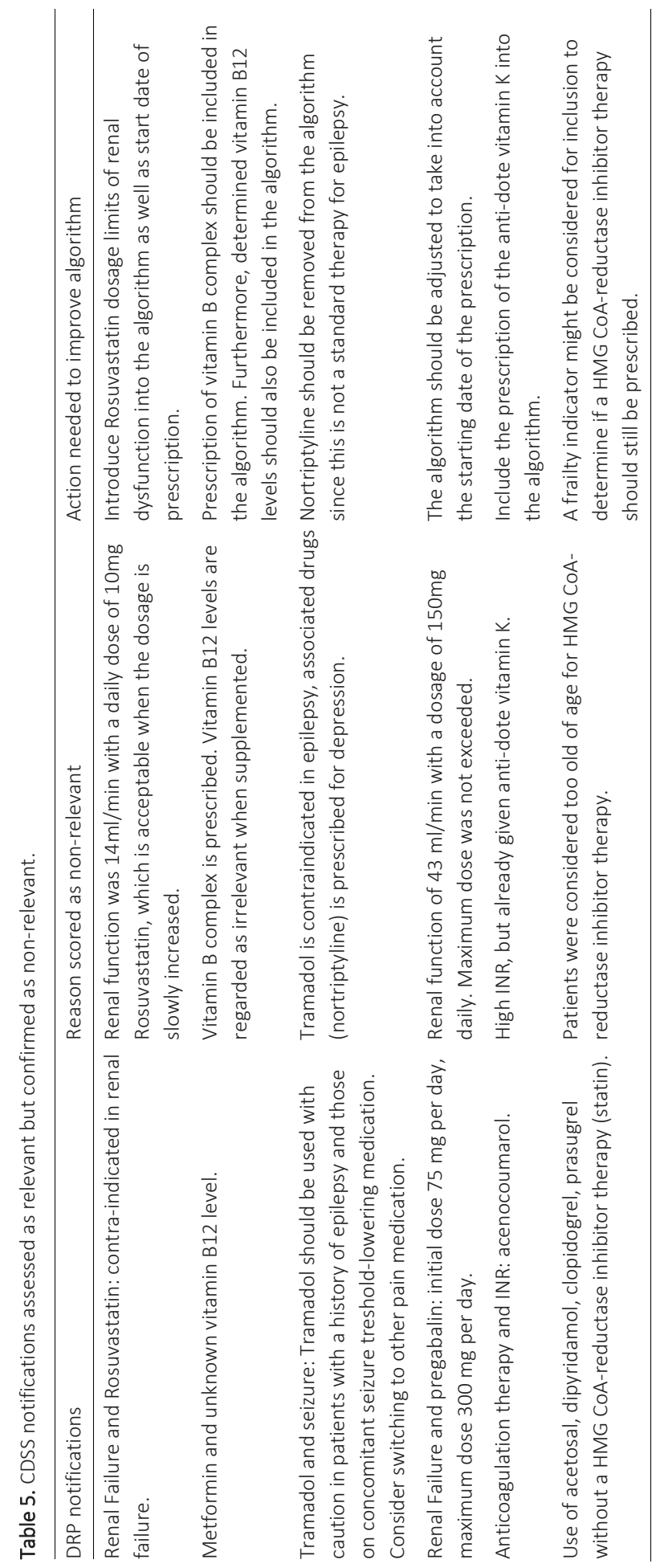




\section{CDSS supports medication review}

Despite the absence of conclusive benefits for morbidity and mortality, medication reviews feature prominently in pharmacists' and physicians' daily work $(13,12,30,10)$. Many other studies have shown improvement of medication safety in elderly with the support of a CDSS $(31,32)$. Whether a CDSS can support the manual medication review can be shown by comparing the confirmed DRP notifications with the DRP strategies of the medication reviews. This study shows that $20 \%$ of the DRP strategies were also identified by the CDSS. Furthermore, the CDSS identified 26 DRPs that were overlooked in the manual medication review showing that the CDSS adds value to performing the medication review manually. Meulendijk et al. also suggested that a CDSS may improve medication review effectiveness (21). However, the results also show the CDSS can be improved in efficiency and content.

\section{CDSS strengths and weaknesses}

The classification of types of medication errors shows that the notifications prompted by the CDSS, which went unrecognised during the medication reviews are strongly represented by the medication error types (a) 'indication without medication' and error type (c) 'contraindications/interactions/side effects'. The importance in recognising these medication error types has also been shown by Leendertse et al. in the evaluation of the potential causes of the preventable medication-related hospital admissions. Their evaluation showed a variety of a number of potential causes like gastrointestinal problems such as bleeding and constipation and cardiovascular problems such as heart failure (6). These cases also involved the medication error types (a) 'indication without medication', and (c) 'contraindications/interactions/side effects', mostly missed in the medication review but recognised by the CDSS.

The medication error types (b) 'medication without indication', (e) 'double medication', and (f) 'wrong medication' were frequently not identified by our CDSS. Depending on the medication error type, it can be reasoned why the CDSS did not find these types of errors. The medication error type (b) requires the input of the 'indication'. In the included medication reviews only three indications for all patients where documented in the CPOE and therefore available for the CDSS. It has already been suggested that multi-morbidity from electronic health records is poorly adopted by currents CDSSs (33). The reason why the medication error types (e) 'double medication' and (f) 'wrong medication' were not found by the CDSS is because these DRPs are not included in the algorithms. These DRPs are routinely screened by the physician and pharmacist in the CPOE integrated G-standard. Our CDSS has been developed to have new notifications in addition to the CPOE notifications. The appearance of the many medication error types (e) 'double medication' and (f) 'wrong medication', suggests there is a high degree of 


\section{Chapter 5}

alert fatigue with regard to CPOE alerts. Alert fatigue occurs when there are a high number of non-clinically relevant alerts, which results in the overlooking of both relevant and not-relevant alerts (34). The CPOE integrated G-standard has been reported to generate $5.8 \%$ relevant drug safety alerts (17). The efficiency of our CDSS is much higher with a sensitivity of $72.9 \%$ and a specificity of $98.6 \%$. The analysis of why the CDSS assessed DRP notifications as non-relevant or relevant, while later evaluated as relevant and non-relevant respectively, can be used to increase the efficiency of the CDSS.

\section{Limitations of the study}

We attempted to estimate the added value of the developed CDSS and the effectiveness of the manual medication review. Our study was limited, however, to the performed medication reviews, the CDSS-generated DRP notifications and the DRPS executed within a week after the medication review. An analysis of why DRP strategies were not executed would perhaps have provided interesting insights into the execution part of the medication review process. Furthermore, all DRPs were classified according to the type of medication error observed. A few DRPs, however, involved consultations with other physicians concerning the use of certain drugs or involved reminders to evaluate the necessity of certain drugs. We classified these DRPs as relevant, since there was considerable doubt related to the chosen therapy. This might be interpreted differently in other studies.

\section{Further research}

Using automation to make medication reviews more efficient is considered highly necessary according to a recent survey (12). There remain several differences between CDSSs both content and efficiency of current CDSS varies considerably. Some CDSSs still rely on the manual input of a single patient's details such as medical history, medication and pathology. These CDSSs do support the medication review but are lacking in terms of time efficiency (35). The effectiveness of our CDSS to support medication reviews needs to be increased by 1) complementing the content with overlooked DRP strategies from the medication review and 2) by optimising the DRP notifications that were incorrectly assessed by the CDSS. Furthermore, the results of this study show that certain DRP notifications are correct when strictly following the applicable guideline, but are found to be irrelevant after discussion in the gerontopharmacology meeting. The development of algorithms allowing discriminating between patients to initiate 'deprescribing' or to specifically not initiate pharmacotherapy treatment will be a challenge. Deprescribing aims to reduce the use of drugs that are less beneficial, or even, detrimental taking into account the individual needs for therapy (36). The challenge of incorporating 'deprescribing' is shown by the lack of DRP notifications in medication error type (b) 'medication without indication'. An example of deprescribing and of not initiat- 
ing pharmacotherapy treatment is the prescription of statins. In our study, treatment with HMG CoA-reductase inhibitor (statins) was not initiated in ten patients because they were too frail and too old. The benefit of stopping statins or not initiating statins when life expectancy is limited should be considered in CDSSs (37). The development of algorithms approaching the level of expertise in the gerontopharmacology meeting will be a great challenge and improvement for the CDSS.

\section{CONCLUSIONS}

Performing medication reviews in a hospitalised geriatric patient group can be of value seen the high number of accepted DRP strategies. More than half of the accepted DRP strategies were executed within one week. The distribution of the relevant medication error types shows the strengths and weaknesses of the CDSS compared to the manual medication review in this study. Our developed CDSS is currently unable to replace the manual medication review. It can however be of additional value for the manual medication review. Further development of the current CDSS is needed to fully support manual medication reviews. 


\section{REFERENCES}

1. Clegg A, Young J, Iliffe S, Rikkert MO, Rockwood K. Frailty in elderly people. Lancet. 2013;381(9868):752-62.

2. Maher RL, Hanlon J, Hajjar ER. Clinical consequences of polypharmacy in elderly. Expert Opin Drug Safety. 2014;13(1):57-65.

3. Hajjar ER, Cafiero AC, Hanlon JT. Polypharmacy in elderly patients. Am J Geriatr Pharmacother. 2007;5(4):345-51.

4. Herr M, Robine JM, Pinot J, Arvieu JJ, Ankri J. Polypharmacy and frailty: prevalence, relationship, and impact on mortality in a French sample of 2350 old people. Pharmacoepidemiol Drug Safety. 2015;24(6):637-46.

5. Silva C, Ramalho C, Luz I, Monteiro J, Fresco P. Drug-related problems in institutionalized, polymedicated elderly patients: opportunities for pharmacist intervention. Int J Clin Pharm. 2015;37(2):327-34.

6. Leendertse AJ, Egberts AC, Stoker LJ, van den Bemt PM, Group HS. Frequency of and risk factors for preventable medication-related hospital admissions in the Netherlands. Arch Intern Med. 2008;168(17): 1890-6.

7. Nobili A, Licata G, Salerno F, Pasina L, Tettamanti M, Franchi C et al. Polypharmacy, length of hospital stay, and in-hospital mortality among elderly patients in internal medicine wards. The REPOSI study. Eur J Clin Pharmacol. 2011;67(5):507-19.

8. Blenkinsopp A, Bond C, Raynor DK. Medication Reviews. Br J Clin Pharmacol. 2012;74(4):573-80.

9. van Dijk KN, van Asselt DZ, Vogel D, van der Hooft CS, Van Roon EN, Brouwers JR. Effects of a structured medication review by geriatrician and clinical pharmacologist on appropriateness of pharmacotherapy in frail elderly inpatients. Tijdschr Gerontol Geriatr. 2009;40(2):72-8.

10. Christensen $\mathrm{M}$, Lundh $\mathrm{A}$. Medication review in hospitalised patients to reduce morbidity and mortality. Cochrane Database Syst Rev. 2013;2:CD008986.

11. Alldred DP, Raynor DK, Hughes C, Barber N, Chen TF, Spoor P. Interventions to optimise prescribing for older people in care homes. Cochrane Database Syst Rev. 2013;2:CD009095.

12. de Wit HAJM, Winkens B, Mestres Gonzalvo C, Hurkens KPGM, Janknegt R, Schols JMGA et al. Clinical practice of medication reviews in institutional care settings for older people in the Netherlands: an explorative survey. Eur J Hosp Pharm. 2015;22(4):212-218

13. Hurkens KPGM, Mestres Gonzalvo C, de Wit HAJM, van der Kuy P-HM, Janknegt R, Verhey FR et al. A Survey on Medication Reviews in Older Patients: Substantial Variation in Daily Practice. J Gerontol Geriat Res 2013;2(133).

14. Mestres Gonzalvo C, Hurkens KPGM, de Wit HAJM, van Oijen BPC, Janknegt R, Schols JMGA et al. To what extent is clinical and laboratory information used to perform medication reviews in the nursing home setting? the CLEAR study. Ther Clin Risk Manag. 2015;11:767-77.

15. de Wit HAJM, Mestres Gonzalvo C, Hurkens KP, Mulder WJ, Janknegt R, Verhey FR et al. Development of a computer system to support medication reviews in nursing homes. Int J Clin Pharm. 2013;35(5):668-72.

16. KNMG. Richtlijn elektronisch voorschrijven [Internet]. 2013. [ cited on 20 Sep 2015] Available from: http://knmg.artsennet.nl/Publicaties/KNMGpublicatie/136411/Richtlijn-elektronisch-voorschrijven-2013.htm.

17. Eppenga WL, Derijks HJ, Conemans JM, Hermens WA, Wensing M, De Smet PA. Comparison of a basic and an advanced pharmacotherapy-related clinical decision support system in a hospital care setting in the Netherlands. J Am Med Inform Assoc. 2012;19(1):66-71.

18. Kuperman GJ, Bobb A, Payne TH, Avery AJ, Gandhi TK, Burns G et al. Medication-related clinical decision support in computerized provider order entry systems: a review. J Am Med Inform Assoc. 2007;14(1):29-40.

19. Rommers MK, Teepe-Twiss IM, Guchelaar HJ. A computerized adverse drug event alerting system using clinical rules: a retrospective and prospective comparison with conventional medication surveillance in the Netherlands. Drug Saf. 2011;34(3):233-42.

20. O'Sullivan D, O'Mahony D, O'Connor MN, Gallagher P, Cullinan S, O'Sullivan R et al. The impact of a structured pharmacist intervention on the appropriateness of prescribing in older hospitalized patients. Drugs Aging. 2014;31(6):471-81. 
21. Meulendijk MC, Spruit MR, Drenth-van Maanen AC, Numans ME, Brinkkemper S, Jansen PA et al. Computerized Decision Support Improves Medication Review Effectiveness: An Experiment Evaluating the STRIP Assistant's Usability. Drugs Aging. 2015;32(6):495-503.

22. de Wit HAJM, Mestres Gonzalvo C, Cardenas J, Derijks HJ, Janknegt R, van der Kuy PH et al. Evaluation of clinical rules in a standalone pharmacy based clinical decision support system for hospitalized and nursing home patients. Int J Med Inform. 2015;84(6):396-405.

23. Tawadrous D, Shariff SZ, Haynes RB, lansavichus AV, Jain AK, Garg AX. Use of clinical decision support systems for kidney-related drug prescribing: a systematic review. Am J Kidney Dis. 2011;58(6):903-14.

24. Bright TJ, Wong A, Dhurjati R, Bristow E, Bastian L, Coeytaux RR et al. Effect of clinical decision-support systems: a systematic review. Ann Intern Med. 2012;157(1):29-43.

25. Jaspers MW, Smeulers M, Vermeulen H, Peute LW. Effects of clinical decision-support systems on practitioner performance and patient outcomes: a synthesis of high-quality systematic review findings. J Am Med Inform Assoc. 2011;18(3):327-34.

26. Schols JM, Crebolder HF, van Weel C. Nursing home and nursing home physician: the Dutch experience. Journal of the American Medical Directors Association. 2004;5(3):207-12.

27. Leendertse AJ, de Koning FH, Goudswaard AN, Jonkhoff AR, van den Bogert SC, de Gier HJ et al. Preventing hospital admissions by reviewing medication (PHARM) in primary care: design of the cluster randomised, controlled, multi-centre PHARM-study. BMC Health Serv Res. 2011;11:4.

28. Verrue $\mathrm{CL}$, Petrovic M, Mehuys E, Remon JP, Vander Stichele R. Pharmacists' interventions for optimization of medication use in nursing homes : a systematic review. Drugs Aging. 2009;26(1):37-49.

29. Mestres C, Agustí A, Puerta L, Barba M. Prescription of potentially inappropriate drugs for geriatric patients in long-term care: improvement through pharmacist's intervention. Eur J Hosp Pharm. 2015;22(4):198-201.

30. Wallerstedt SM, Kindblom JM, Nylen K, Samuelsson O, Strandell A. Medication reviews for nursing home residents to reduce mortality and hospitalization: systematic review and meta-analysis. $\mathrm{Br} \mathrm{J} \mathrm{Clin}$ Pharmacol. 2014;78(3):488-97.

31. Marasinghe KM. Computerised clinical decision support systems to improve medication safety in longterm care homes: a systematic review. BMJ open. 2015;5(5):e006539.

32. Ranji SR, Rennke S, Wachter RM. Computerised provider order entry combined with clinical decision support systems to improve medication safety: a narrative review. BMJ quality \& safety. 2014;23(9):773-80.

33. Fraccaro P, Arguello Casteleiro M, Ainsworth J, Buchan I. Adoption of clinical decision support in multimorbidity: a systematic review. JMIR medical informatics. 2015;3(1):e4.

34. van der Sijs H, Aarts J, Vulto A, Berg M. Overriding of drug safety alerts in computerized physician order entry. J Am Med Inform Assoc. 2006;13(2):138-47.

35. de Wit HAJM, Mestres Gonzalvo C, Janknegt R, Schols JM, van der Kuy PH. A fully automated medication review? Int J Clin Pharm. 2014;36(2):220-1.

36. Scott IA, Hilmer SN, Reeve E, Potter K, Le Couteur D, Rigby D et al. Reducing inappropriate polypharmacy: the process of deprescribing. JAMA internal medicine. 2015;175(5):827-34.

37. Holmes HM, Todd A. Evidence-based deprescribing of statins in patients with advanced illness. JAMA internal medicine. 2015;175(5):701-2. 

The development of an automated ward independent delirium risk prediction model

This chapter is based on:

de Wit HAJM, Winkens B, Mestres Gonzalvo C, Hurkens KPGM, Mulder WJ, Janknegt R, Verhey FR, van der Kuy PHM, Schols JMGA.

The development of an automated ward independent delirium risk prediction model. Int J Clin Pharm. 2016;38(4):915-23. 


\section{Chapter 6}

\section{ABSTRACT}

\section{Introduction}

A delirium is common in hospital settings resulting in increased mortality and costs. Prevention of a delirium is clearly preferred over treatment. A delirium risk prediction model can be helpful to identify patients at risk of a delirium, allowing the start of preventive treatment. Current risk prediction models rely on manual calculation of the individual patient risk. The aim of this study was to develop an automated ward independent delirium risk-prediction model and to show that such a model can be constructed exclusively from electronically available risk factors and thereby implemented into a clinical decision support system (CDSS) to optimally support the physician to initiate preventive treatment.

\section{Methods}

The study involved a retrospective cohort study in which patients, 60 years or older, were selected when admitted to the hospital, with no delirium diagnosis when presenting, or during the first day of admission. We used logistic regression analysis to develop a delirium predictive model out of the electronically available predictive variables.

\section{Results}

A delirium risk prediction model was developed using electronically available predictive variables that were significant in the univariable regression analyses. The AUROC (area under the receiver operating characteristics curve) of the 'medication model' model was 0.76 after internal validation.

\section{Conclusions}

CDSSs can be used to automatically predict the risk of a delirium in individual hospitalised patients' by exclusively using electronically available predictive variables. To increase the use and improve the quality of predictive models, additional, relevant clinical risk factors should be documented ready for automated use. 


\section{INTRODUCTION}

A delirium is a multifactorial acute brain dysfunction common in multimorbid, frail and disabled older adults and a sign of the brain's vulnerability to external factors (1). The prevalence of delirium at hospital admission is reported to be 10 to $31 \%$ whereas the incidence of a new delirium during admission is 3 to $29 \%$ (2). Delirium is associated with high costs ranging from US \$16 303 to $\$ 64421$ per patient (3). Also, hospital mortality rates are high, ranging from $25 \%$ to $33 \%$. A hospital admission with a delirium episode gives an independent increased risk for death $(1,4)$.

Inouye et al. have shown that using protocols in the management of delirium risk factors can reduce the incidence of delirium, as well as the number of delirium days and delirium episodes (5). Therefore, screening vulnerable patients for delirium is important to allow for (early) diagnosis and treatment. Several screening methods for delirium assessment are available such as the Confusion Assessment Method (CAM), and the Nursing Delirium Screening Scale (Nu-DESC). These two methods are considered to be the most adequate as diagnostic tools to initiate the management of delirium symptoms and treat the underlying medical condition $(6,7)$. Prevention of delirium is clearly preferred over treatment. Prevention of delirium is possible, using pharmacological and non-pharmacological treatment $(8,9)$. Preventive interventions have shown to be effective, e.g. peri-operative use of antipsychotics in older adults at risk may reduce the incidence of a delirium and a reduction of severity and length of the delirium with haloperidol prophylaxis can be achieved $(10,11)$. However, a recent systematic review concluded that robust evidence is lacking to support the broad use haloperidol for inhospital delirium. This meta-analysis has shown that non-pharmacological interventions decrease the occurrence of delirium in hospitalised older adults (12). Therefore, nonpharmacological interventions, such as (re)orientation in time and place, family visits, optimising nutrition status, and improvement of sensory impairment remain important measures to prevent a delirium $(8,13)$.

\section{Delirium prediction}

Risk factors associated with delirium are categorised into predisposing and precipitating factors. Predisposing factors can be: age, cognitive disorders, illness severity (using severity-of-disease classification systems such as the 'Acute Physiology and Chronic Health Evaluation II' or a trichotomised nurse rate scale), Barthel Index (an index to measure activities of daily living), and vision impairment. Precipitating factors can be: alcohol use, infections, fever, dehydration, and certain drugs. Specific drug properties can also cause a delirium, but in general these effects are reversible $(8,14-18)$. A delirium risk predictive model uses risk factors to identify patients at risk of a delirium. Such predictive models already exist for intensive care patients and for hospitalised older adults and include different precipitating and predisposing factors as predictive variables, however they rely 


\section{Chapter 6}

on the manual calculation of the patient's risk $(16,17,19,20)$. For the practical use of these predictive models they are sometimes simplified to risk stratification models. An automated delirium prediction model that calculates risks for delirium in all hospitalised patients will support the physician more efficiently to identify these patients and to initiate timely treatment. Such a model can only be applied in practice if it relies exclusively on electronically available risk factors. A system that combines electronically available risk factors, such as medication and laboratory values, is known as a clinical decision support system (CDSS). A CDSS can provide around the clock surveillance and can integrate different algorithm types such as risk prediction models and clinical management tables (21). Although some important risk factors used in delirium predictive models are not electronically available yet, developing a delirium risk prediction model using exclusively electronically available risk factors might already be useful.

\section{AIM OF THE STUDY}

The aim of this study was to develop a ward independent delirium risk prediction model for the use in a CDSS using exclusively currently electronically available risk factors.

\section{ETHICAL APPROVAL}

No ethical approval from the local medical ethics committee was needed since only anonymised hospital data were used.

\section{METHODS}

This study in which a delirium risk prediction model was developed was a retrospective cohort study executed in Zuyderland Medical Centre, Sittard, The Netherlands.

\section{Study population}

Patients of 60 years or older, admitted to the hospital between 2008 and 2012, were included in the study. In order to include as many patients as possible we chose a cutoff point of 60 years or older, which is the lowest cut-off point of delirium associated in a geriatric population $(13,15,22)$. Patients admitted with or diagnosed with a delirium on the first day of admission were excluded. Initially, 646 delirium documentations were retrieved from the 'diagnosis table' in the electronic patient's records (EPR). The 'diagnosis table' is where the physician can enter diagnoses using 'plain text', but at this moment cannot use the International Classification of Diseases and Related Health 
Problems (ICD-10). The actual date of delirium diagnosis during admission was manually retrieved from the 'plain text' EPR, which also confirmed that the patient indeed had a delirium episode during admission. Of these 646 delirium documentations, 421 were excluded as a result of double delirium documentation in the same patient, delirium at the moment of hospital admission, delirium on the first day of admission, or age below 60 years. The control patients did not have a delirium episode documented in the 'diagnosis table' of the EPR. Control patients were selected based on a randomly chosen hospital admission day and an age of 60 years or older. Matching on 'gender' or 'age' was not used since these are predictive variables in the model. The first day of each month was a randomly chosen day of hospital admission for the inclusion of the control patients, thereby taking into account an equal distribution of the number of control patients per calendar year compared to the number of delirium patients in the same calendar year.

\section{Potential Predictive Variables}

Risk factors were included into the database when fulfilling three criteria: 1) previously identified in the literature, 2) available for data extraction from the hospital EPR, and 3) ready for automation in a CDSS. Twenty-six risk factors were identified according to these criteria and included in the database. The risk factors were: age, gender, polypharmacy, use of anxiolytics, antidepressants, anti-dementia medication, antiparkinson medication, antihistaminics, antidiabetics, psychopharmaca, analgesics, sleep medication, laboratory values ALT (serum alanine transaminase), AST (serum aspartate transaminase), GGT (serum gamma-glutamyl transferase), creatinine, C-reactive protein (CRP), glucose, potassium, urea (the highest value), alkaline reserve as a value for metabolic acidosis, albumin, sodium, calcium, anion-gap, and hemoglobin (the lowest value) $(1,14,15,19,23)$.

\section{Moment of data extraction}

Considering there is no known time frame to select risk factors for the prediction of a delirium, our predictive variables were extracted between 1 to 3 days before the delirium diagnosis. This time frame was selected so that the predictive variables were to be of predictive use and quality. As most laboratory values were not determined daily, the most deviating laboratory values, 1 to 3 days prior to the delirium diagnosis, were used.

The data concerning prescribed drugs were extracted one day before the delirium diagnosis. The 'reference date' for extraction of the control group data was set equal to the median number of hospital admission days until delirium diagnosis in the delirium patients in this study. The median was preferred over the mean, since the mean number of days is likely to be inflated by outliers (high number of hospital days until delirium diagnosis). 


\section{Chapter 6}

\section{Data extraction from hospital systems}

The predictive variables were classified into two groups depending on the system from which they were extracted; 1) computerised physician order entry (CPOE) extracted predictive variables and 2) laboratory values as predictive variables. The CPOE system for prescribing medication used in the Zuyderland Medical Centre, facilitated the electronic extraction of the predictive variables age, gender, polypharmacy, prescription of anxiolytics, antidepressives, anti-dementia medication, antiparkinson medication, antihistaminics, antidiabetics, analgetics, and sleep medication. These known risk factors were inferred as predictive variables by extracting them from the CPOE system according to the Anatomical Therapeutic Chemical (ATC) classification system. Using the ATC classification system the prescribed drugs could be extracted according to the known drug classes (24). The risk factor 'diabetes mellitus' for delirium was included by correlating it to the prescription of an anti-diabetic drug. The risk factor 'polypharmacy' accounts for the number of prescribed drugs. Polypharmacy can be defined as the use of a higher number of drugs than clinically necessary, but is often defined by using an arbitrary cut-off point ranging from more than two to seven drugs per patient $(25,26)$. We have therefore included polypharmacy as a predictive variable by counting the number of prescribed drugs per patient. The laboratory values, for both the delirium and the control group, were extracted between 1 to 3 days before the delirium diagnosis (or reference date).

\section{Statistical analysis}

It was anticipated that the predictive model would include 10 to a maximum of 20 risk factors. To ensure at least 10 delirium patients per risk factor used in the predictive model a minimum of 200 delirium patients were needed (27). With an expected incidence of $3-29 \%$ new deliriums after admission, we aimed to include the number of control patients that ensures an average incidence of about $16 \%$ (2).

To construct the delirium risk prediction model from the database, a two-step procedure was used. First, univariable logistic regression analysis was applied to the data, and only those variables having a p-value $\leq 0.05$ were included in the multivariable model. Missing values were imputed by multiple imputations (MI), creating five complete datasets. Linearity assumption was checked for numerical variables by adding and testing centred quadratic terms. Secondly, the multivariable model was applied to determine the weight of the included predictive variables.

The optimal cut-off point of the model was determined from the receiver operating characteristics (ROC) curve, i.e. the point closest to the upper left corner, which minimises the sum of (1-sensitivity) ${ }^{2}$ and (1-specificity) ${ }^{2}$ (28). Since laboratory values are not always available, another model that included only the patient's characteristics and medication as predictive variables was developed. This limited 'medication' model was 
developed as a sensitivity analysis for the 'full' model, using the same two step procedure, but multiple imputation was not necessary. IBM SPSS for windows (version 21.0) was used for analyses. Two-sided $p$-values $\leq 0.05$ were considered statistically significant. Since the predictive quality of the model might be overestimated due to overfitting, an internal validation (1000 bootstrapping samples) of the 'medication' model was performed using the 'rms' package within R software (version 3.2.3) (29).

\section{RESULTS}

Of the 646 delirium patients available from the EPR, 225 patients were included as the index group in the database. Another 1066 patients were included as the control group. The delirium incidence in the database was $17.4 \%$ which is acceptable. The patients' characteristics are shown in table 1.

Of the twenty-six potential predictive variables, twelve and ten predictive variables were included in the 'full' and 'medication' model, respectively. Predictive variables that were left out of the 'full' model were: gender, antihistamine use (ATC R06) and all laboratory values except for the CRP and urea.

Table 1. Patients' characteristics.

\begin{tabular}{llll}
\hline & Delirium $(\mathrm{n}=225)$ & Control $(\mathrm{n}=1066)$ & Total $(\mathrm{n}=1291)$ \\
\hline Mean age (years) & $81.2($ SD 7.6$)$ & $76.0($ SD 7.8$)$ & $76.9($ SD 8.0$)$ \\
Male (patients) & $113(50.2 \%)$ & $555(52.1 \%)$ & $668(51.7 \%)$ \\
Female (patients) & $112(49.8 \%)$ & $511(47.9 \%)$ & $623(48.3 \%)$ \\
\hline
\end{tabular}

The median delirium diagnosis was observed at three days (ranging from 1 to 141 days) after hospital admission, and was used as the 'reference date' for the extraction of the predictive variables in the control group.

The two laboratory values included in the 'full' model, urea and CRP, had 353 (27.3\%) and 396 (30.7\%) missing values from all 1291 patients, respectively. After accounting for the missing data by applying $\mathrm{MI}$, the prediction quality of the multivariable model did not vary much between the five datasets. The area under the ROC curves' (AUROC) ranged from 0.77 to $0.78(95 \% \mathrm{Cl} 0.74$ to 0.81$)$. The same applies to the optimal cut-off points (ranging from $13.7 \%$ to $14.8 \%$ ). The corresponding sensitivity was relatively high, ranging from $78.2 \%$ to $80 \%$, whereas the specificity was lower $(61.9 \%$ to $65.3 \%)$. Figure 1 a shows the ROC closest to the mean AUROC $(=0.78)$. The other ROC curves were similar. The optimal cut-off point obtained from the ROC was $14.2 \%$, resulting in a sensitivity of $78.2 \%$, a specificity of $63.5 \%$, and a positive and negative likelihood ratio of 2.14 and 0.34 , respectively. 


\section{Chapter 6}

\section{Sensitivity analysis}

As a sensitivity analysis, the 'medication' model was developed to assess whether using the laboratory variables would increase the predictive quality of the model. The 'medication' model resulted in an AUROC of 0.77 (95\% Cl 0.74 to 0.80$)$, where the optimal cut-off point is $14.1 \%$ (see figure $1 \mathrm{~b}$ ), resulting in a sensitivity of $78.2 \%$ and a specificity of $63.7 \%$ and a positive and negative likelihood ratio of 2.15 and 0.34 , respectively. After correcting for overfitting (internal validation), the AUROC decreased only slightly to 0.76 . The formulas of the 'full' model and the 'medication' model that allows for calculating the individual risk are shown in table 2.

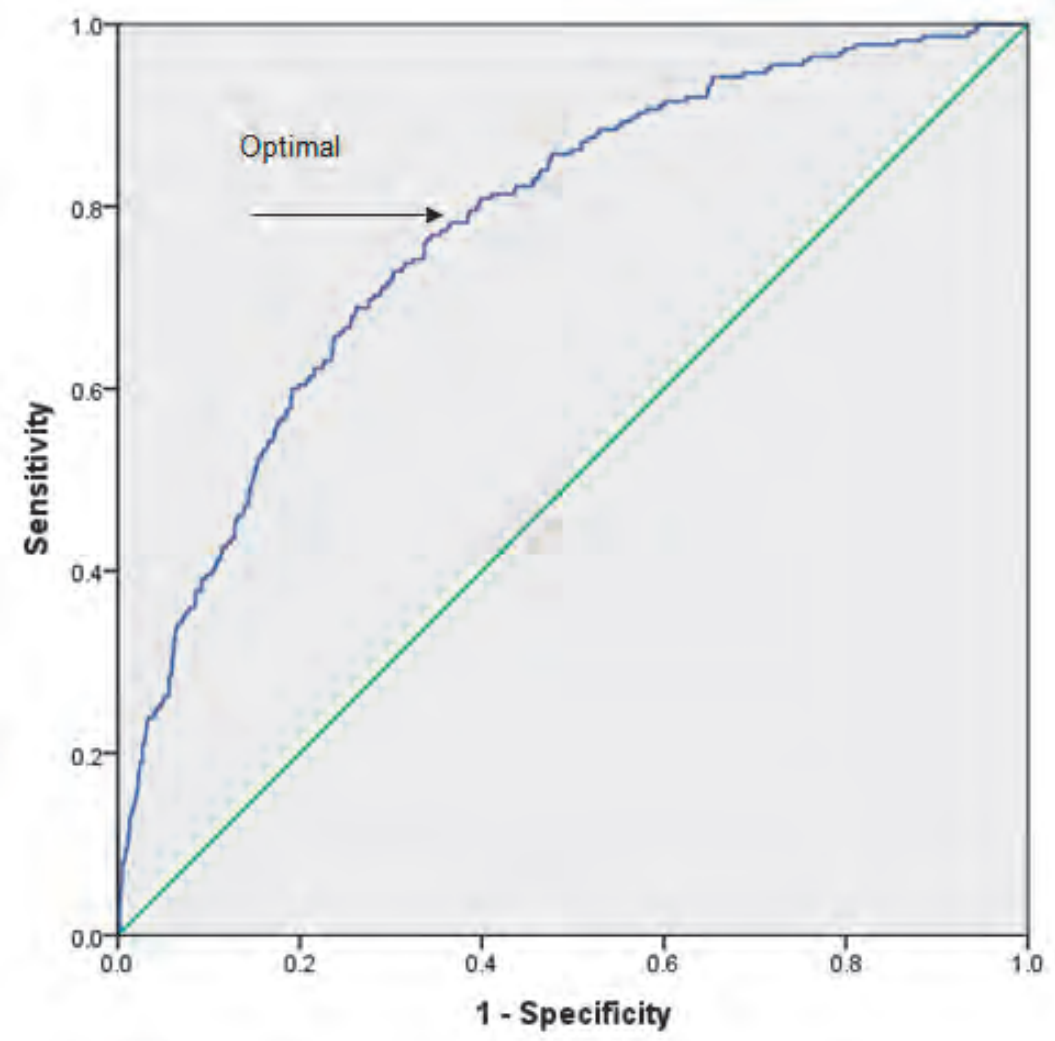

Figure 1a: Receiver operating characteristics (ROC)-curve closest to the mean AUROC of the full predictive delirium model. 
The development of an automated ward independent delirium risk prediction model

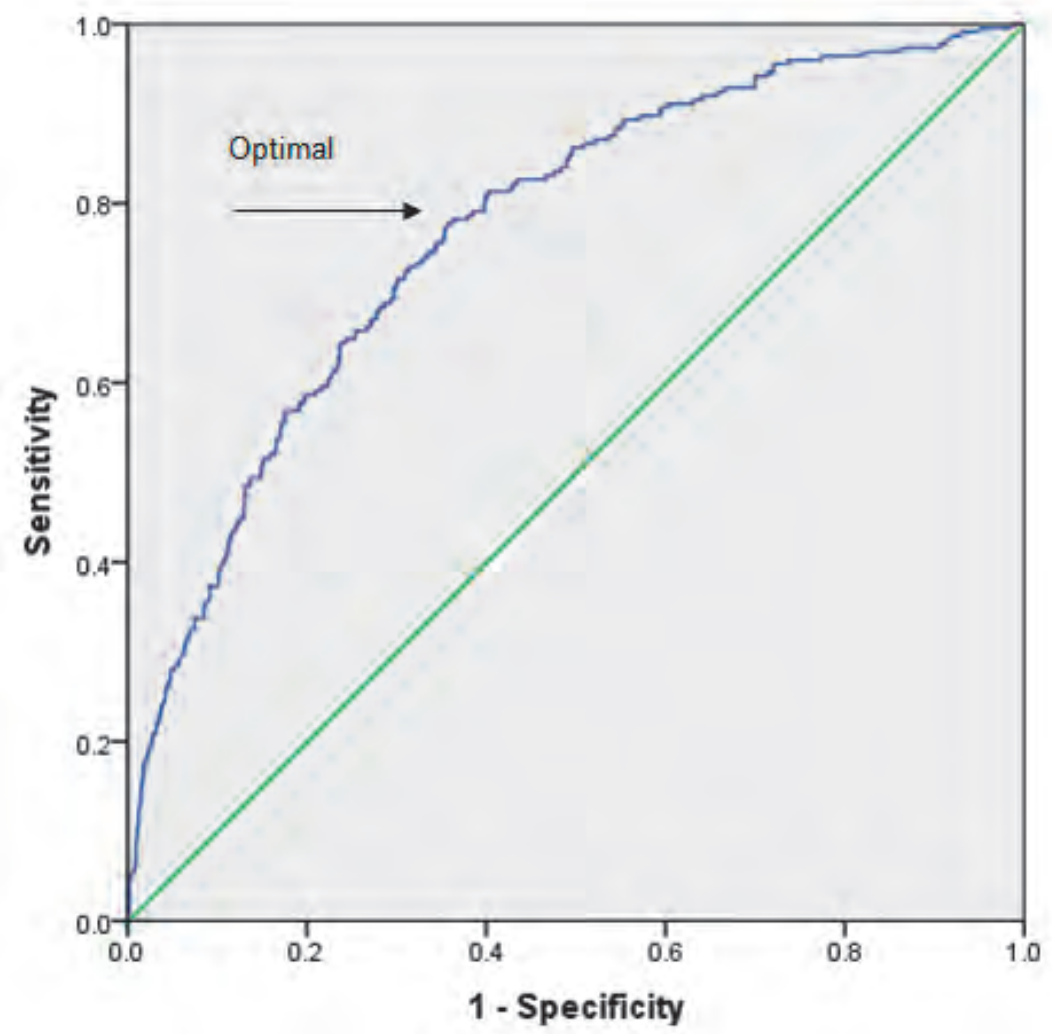

Figure 1b: Receiver operating characteristics (ROC)-curve of the 'medication' predictive delirium model. 


\section{Chapter 6}

Table 2. Two models to calculate the individual risk of developing a delirium in patients older than 60 years: the 'full model' and the 'medication model'. Appendix A shows the complete regression table.

Formula Individual delirium risk:

$$
=1 /\left(1+\mathrm{e}^{-(\underline{\text { Linear predictor })})}\right.
$$

Linear predictor full model $=$

$$
\begin{aligned}
& -9.118+(0.082 * \mathrm{~V} 1)+(0.029 * \mathrm{~V} 2)+(0.248 * \mathrm{~V} 3)+(1.123 * \mathrm{~V} 4)+(0.286 * \mathrm{~V} 5)+(1.962 * \mathrm{~V} 6)+(0.330 * \mathrm{~V} 7) \\
& +(1.164 * \mathrm{~V} 8)+(0.371 * \mathrm{~V} 9)+(0.155 * \mathrm{~V} 10)+(0.002 * \mathrm{~V} 11)+(-0.001 * \mathrm{~V} 12)
\end{aligned}
$$

Linear predictor medication model $=$

$$
\begin{aligned}
& -8.823+(0.081 * \mathrm{~V} 1)+(0.031 * \mathrm{~V} 2)+(0.173 * \mathrm{~V} 3)+(1.116 * \mathrm{~V} 4)+(0.266 * \mathrm{~V} 5)+(1.923 * \mathrm{~V} 6)+(0.359 * \mathrm{~V} 7) \\
& +(1.199 * \mathrm{~V} 8)+(0.413 * \mathrm{~V} 9)+(0.103 * \mathrm{~V} 10)
\end{aligned}
$$

Predictive variables used:

V1. Age (years)

V2. Polypharmacy ATC- $5^{\text {th }}$

Use of medication (yes $=1$, no $=0$ ):

$\begin{array}{cll}\text { V3. } & \text { Anxiolytics } & \text { N05B } \\ \text { V4. } & \text { Anti-dementia } & \text { N06D } \\ \text { V5. } & \text { Antidepressives } & \text { N06A } \\ \text { V6. } & \text { Antiparkinson's } & \text { N04 } \\ \text { V7. } & \text { Antidiabetic's } & \text { A10 } \\ \text { V8. } & \text { Psychopharmaca } & \text { N05A } \\ \text { V9. } & \text { Analgetics } & \text { N02A } \\ \text { V10. } & \text { Sleepmedication } & \text { N05C }\end{array}$

Value of laboratory result:

V11. CRP (mg/l)

V12. Urea $(\mathrm{mmol} / \mathrm{l})$

\section{DISCUSSION}

\section{Clinical relevance}

In this study we developed a hospital-wide, ward independent, delirium prediction model in older patients using individual risk calculation. With this model, we demonstrated that a complex prediction model, like delirium risk prediction, can be automated by exclusively using electronically available risk factors. This shows that a CDSS can be used to automatically calculate the individual patient's delirium risk in hospitalised patients.

The sensitivity analysis with the 'medication' model shows that delirium can also be effectively predicted when only the prescribed medication and age are available as predictive variables. The addition of laboratory variables to the model resulted in a marginal increase of the AUROC from 0.77 to 0.78 , which will not make much difference to the prediction quality of the model. After internal validation (1000 bootstrapping 
samples) of the 'medication model' the AUROC only decreased slightly to 0.76 , which shows that the predictive value was not largely overestimated.

The use of the predictive variables CRP and urea was not very beneficial, which was unexpected since these variables would have incorporated the onset of an infection and a diminished renal function into the prediction, respectively. The contribution of the variables in the 'medication' model (table 2) can be explained by their individual properties. The fact that increasing age contributes to the model appears to be normal because increasing age makes the brain more vulnerable to external factors (1). Polypharmacy is shown to be an independent risk factor for delirium in older adults which might be explained by the known effect of polypharmacy on a patient's frailty status which is also associated with delirium (30-32). Furthermore, the included drug groups, with the exception of the antidiabetics, exert their (adverse) effects directly in the brain by interfering with neurotransmitters, indicating a certain vulnerability of the brain. Cholinergic deficiencies and dopaminergic excess are frequently linked to delirium, which are also influenced by the drugs included in the model. Antipsychotics are known to have anticholinergic properties and antiparkinson medications are known to have dopaminergic properties. Both influence the brain neurotransmitters associated with delirium. Analgesics, anxiolytics, and sleeping medications exert sedative effects which are also known to have a negative effect on the brain; patients are less orientated and easier confused $(1,9)$. Diabetes mellitus is a known risk factor for delirium after cardiac surgery (22). The use of antidiabetics identifies the patients that are susceptible to metabolic derangements. These patients should be monitored for hypoglycaemia and hyperglycaemia (1).

\section{Clinical Practice}

The developed model can be regarded as clinically relevant since an AUROC higher than 0.75 is regarded as clinically relevant (28). Delirium (preventive) treatment is often limited to patients screened with a manual calculated simplified risk model and patients already diagnosed with a delirium. When implemented into a CDSS our model is able to automatically calculate the individual risk of hospitalised patients older than 60 years to timely start preventive treatment. Considering the individual precipitating and predisposing risk factors, the best available preventive treatment can be chosen such as discontinuing precipitating drugs and initiating non-pharmacological preventive treatment.

\section{Limitations of the study}

We included all patients independently of the subtypes of delirium documented such as the hypoactive and hyperactive delirium. Although these subtypes are known to fluctuate within the course of the disorder, a differentiation might influence the sensitivity and specificity of the model (1). The control patients were included as such when a delirium 


\section{Chapter 6}

episode in the EPR 'diagnosis table' was absent. If included control patients had a delirium episode, which wasn't registered properly in the EPR 'diagnosis table', this may have influenced the model in a negative way resulting in a lower predictive quality.

The risk factors included into the model were limited to the factors electronically available. However, other risk factors such as cognitive disorders, vision impairment, lower functional status (Barthel Index) and illness severity have been shown to be very relevant predictive variables for a delirium prediction model as well $(5,15-17)$. In addition, the risk factors 'urgent admission' and 'diagnosis group' (e.g. surgical vs. neurology patients) have also been reported to be relevant for delirium prediction in intensive care patients (19). Unfortunately, in this study, these risk factors were not electronically available for the use in the CDSS. Another limitation is that proxies for dehydration used in this study were the laboratory value Urea and Creatinin instead of the known significant proxy 'blood urea nitrogen /creatinine ratio' (17). Furthermore, the drug prescriptions included into the database were not further specified when prescribed 'as needed' by the physician. This means that all prescribed drugs were included into the database and thereby the assumption was that these drugs were actually administered. Indeed, when prescribed drugs are not administered but included in the database, this might influence the prediction model. Finally, we internally validated the 'medication model' by bootstrapping 1000 times but we did not validate the model in a different set of patients. The latter validation should be performed before implementing the current model into clinical practice.

\section{Future model improvements}

Stusser et al. suggest that a comprehensive CDSS in which all of a patients' information is integrated could be an important guide for the physician (33). Ideally the CDSS's will be integrated in the EPR or CPOE allowing the system to automatically alert the physician when a patient's individual risk, such as a risk for a delirium or for falling, is high and above a defined cut-off point. However, many of these models lack validation or adequate usage in clinical practice (34). The lack of documentation prepared for automation appears to limit the development, validation and use of automated predictive models. In our study, the manual retrieval of the exact date of the delirium diagnosis was the limiting factor for easy validation. Furthermore, other studies included predisposing factors in the model, such as cognitive impairment, previous occurrence of a delirium, the reason for hospital admission, type of performed surgery, vision impairment, functional status, and severity of illness, but these could not be included in our automated model $(17,19)$. It has already been suggested that further automation is dependent on the degree of computerisation of patients' characteristics. Computerisation implies that patients' information is digital, but when it is merely a digital version of a printed document, automation is less evident $(35,36)$. Another approach of including risk factors is the automatic detection of predictive 'trigger'-words from the EPR. This 
might be words such 'hallucin', 'disorient', or 'mental status' from both physician and nurse charts (37). This technique is however not yet supported by our EPR. Moreover, it is generally known that adequate documentation of symptoms in daily clinical practice up until now has shown to be limited (38). In our opinion risk factors and diagnoses should be documented more carefully, precisely, consistently, completely and purposely for automation thereby enabling the development of automated predictive models.

\section{CONCLUSIONS}

This study has shown that a CDSS can be used to automatically calculate the individual patient's delirium risk in hospitalised patients by developing a ward independent model using exclusively electronic predictive variables. To increase the use and improve the quality of predictive models, the registration of clinical patient information should be improved, and the relevant predictive variables should be structurally documented electronically for automated use. 


\section{REFERENCES}

1. Inouye SK, Westendorp RG, Saczynski JS. Delirium in elderly people. Lancet. 2014;383(9920):911-22.

2. Siddiqi N, House AO, Holmes JD. Occurrence and outcome of delirium in medical in-patients: a systematic literature review. Age Ageing. 2006;35(4):350-64.

3. Leslie DL, Marcantonio ER, Zhang Y, Leo-Summers L, Inouye SK. One-year health care costs associated with delirium in the elderly population. Arch Intern Med. 2008;168(1):27-32.

4. Salluh JI, Wang H, Schneider EB, Nagaraja N, Yenokyan G, Damluji A et al. Outcome of delirium in critically ill patients: systematic review and meta-analysis. BMJ. 2015;350:h2538.

5. Inouye SK, Bogardus ST, Jr., Charpentier PA, Leo-Summers L, Acampora D, Holford TR et al. A multicomponent intervention to prevent delirium in hospitalized older patients. N Engl J Med. 1999;340(9):669-76.

6. Inouye SK, van Dyck CH, Alessi CA, Balkin S, Siegal AP, Horwitz RI. Clarifying confusion: the confusion assessment method. A new method for detection of delirium. Ann Intern Med. 1990;113(12):941-8.

7. van Velthuijsen EL, Zwakhalen SM, Warnier RM, Mulder WJ, Verhey FR, Kempen GI. Psychometric properties and feasibility of instruments for the detection of delirium in older hospitalized patients: a systematic review. Int J Geriatr Psychiatry. 2016; doi: 10.1002/gps.4441.

8. Young J, Leentjens AF, George J, Olofsson B, Gustafson Y. Systematic approaches to the prevention and management of patients with delirium. J Psychosom Res. 2008;65(3):267-72.

9. Inouye SK. Delirium in older persons. N Engl J Med. 2006;354(11):1157-65.

10. Teslyar P, Stock VM, Wilk CM, Camsari U, Ehrenreich MJ, Himelhoch S. Prophylaxis with antipsychotic medication reduces the risk of post-operative delirium in elderly patients: a meta-analysis. Psychosomatics. 2013;54(2):124-31.

11. Kalisvaart KJ, de Jonghe JF, Bogaards MJ, Vreeswijk R, Egberts TC, Burger BJ et al. Haloperidol prophylaxis for elderly hip-surgery patients at risk for delirium: a randomized placebo-controlled study. J Am Geriatr Soc. 2005;53(10):1658-66.

12. Hshieh T, Yue J, Oh E, Puelle M, Dowal S, Travison T et al. Effectiveness of multicomponent nonpharmacological delirium interventions: a meta-analysis. JAMA internal medicine. 2015;175(4):512-20.

13. Young J, Murthy L, Westby M, Akunne A, O'Mahony R, Guideline Development G. Diagnosis, prevention, and management of delirium: summary of NICE guidance. BMJ. 2010;341:c3704.

14. Laurila JV, Laakkonen ML, Tilvis RS, Pitkala KH. Predisposing and precipitating factors for delirium in a frail geriatric population. J Psychosom Res. 2008;65(3):249-54.

15. Inouye SK. Predisposing and precipitating factors for delirium in hospitalized older patients. Dement Geriatr Cogn Disord. 1999;10(5):393-400.

16. Carrasco MP, Villarroel L, Andrade M, Calderon J, Gonzalez M. Development and validation of a delirium predictive score in older people. Age Ageing. 2014;43(3):346-51.

17. Inouye SK, Viscoli CM, Horwitz RI, Hurst LD, Tinetti ME. A predictive model for delirium in hospitalized elderly medical patients based on admission characteristics. Ann Intern Med. 1993;119(6):474-81.

18. Young J, Murthy L, Westby M, Akunne A, O'Mahony R. Diagnosis, prevention, and management of delirium: summary of NICE guidance. BMJ. 2010;341.

19. van den Boogaard M, Pickkers P, Slooter AJ, Kuiper MA, Spronk PE, van der Voort PH et al. Development and validation of PRE-DELIRIC (PREdiction of DELIRium in ICu patients) delirium prediction model for intensive care patients: observational multicentre study. BMJ. 2012;344:e420.

20. Douglas VC, Hessler CS, Dhaliwal G, Betjemann JP, Fukuda KA, Alameddine LR et al. The AWOL tool: derivation and validation of a delirium prediction rule. J Hosp Med. 2013;8(9):493-9.

21. de Wit HA, Mestres Gonzalvo C, Hurkens KP, Mulder WJ, Janknegt R, Verhey FR et al. Development of a computer system to support medication reviews in nursing homes. Int J Clin Pharm. 2013;35(5):668-72.

22. Lin $Y$, Chen J, Wang Z. Meta-analysis of factors which influence delirium following cardiac surgery. J Card Surg. 2012;27(4):481-92. 
23. Smulter N, Lingehall HC, Gustafson Y, Olofsson B, Engstrom KG. Delirium after cardiac surgery: incidence and risk factors. Interact Cardiovasc Thorac Surg. 2013;17(5):790-6.

24. WHO [Internet][ last updated: 2015-12-16, cited on 14-10-2015]. Available from: http://www.whocc.no/ atc/structure_and_principles/.

25. Maher RL, Hanlon J, Hajjar ER. Clinical consequences of polypharmacy in elderly. Expert Opin Drug Saf. 2014;13(1):57-65.

26. Hajjar ER, Cafiero AC, Hanlon JT. Polypharmacy in elderly patients. Am J Geriatr Pharmacother. 2007;5(4):345-51.

27. Peduzzi P, Concato J, Kemper E, Holford TR, Feinstein AR. A simulation study of the number of events per variable in logistic regression analysis. J Clin Epidemiol. 1996;49(12):1373-9.

28. Fan J, Upadhye S, Worster A. Understanding receiver operating characteristic (ROC) curves. CJEM. 2006;8(1):19-20.

29. Harrell FE. Regression modeling strategies : with applications to linear models, logistic regression, and survival analysis [Book]. Springer series in statistics. New York: Springer; 2001.

30. Herr M, Robine JM, Pinot J, Arvieu JJ, Ankri J. Polypharmacy and frailty: prevalence, relationship, and impact on mortality in a French sample of 2350 old people. Pharmacoepidemiology and drug safety. 2015;24(6):637-46.

31. Clegg A, Young J, Iliffe S, Rikkert MO, Rockwood K. Frailty in elderly people. Lancet. 2013;381(9868):752-62.

32. Hein C, Forgues A, Piau A, Sommet A, Vellas B, Nourhashémi F. Impact of Polypharmacy on Occurrence of Delirium in Elderly Emergency Patients. J Am Med Dir Assoc. 2014;15(11):850.e11-.e15.

33. Stusser RJ, Dickey RA. Quality and cost improvement of healthcare via complementary measurement and diagnosis of patient general health outcome using electronic health record data: research rationale and design. J Med Sys. 2013;37(6):9977.

34. Minne L, Ludikhuize J, de Rooij SE, Abu-Hanna A. Characterizing predictive models of mortality for older adults and their validation for use in clinical practice. J Am Geriatr Soc. 2011;59(6):1110-5.

35. Soman S, Zasuwa G, Yee J. Automation, Decision Support, and Expert Systems in Nephrology. Adv Chronic Kidney Dis. 2008;15(1):42-55.

36. Maviglia SM, Zielstorff RD, Paterno M, Teich JM, Bates DW, Kuperman GJ. Automating Complex Guidelines for Chronic Disease: Lessons Learned. J Am Med Inform Assoc. 2003;10(2):154-65.

37. Puelle MR, Kosar CM, Xu G, Schmitt E, Jones RN, Marcantonio ER et al. The Language of Delirium: Keywords for Identifying Delirium from Medical Records. J Gerontol Nurs. 2015;41(8):34-42.

38. Hope C, Estrada N, Weir C, Teng CC, Damal K, Sauer BC. Documentation of delirium in the VA electronic health record. BMC Res Notes. 2014;7:208. 


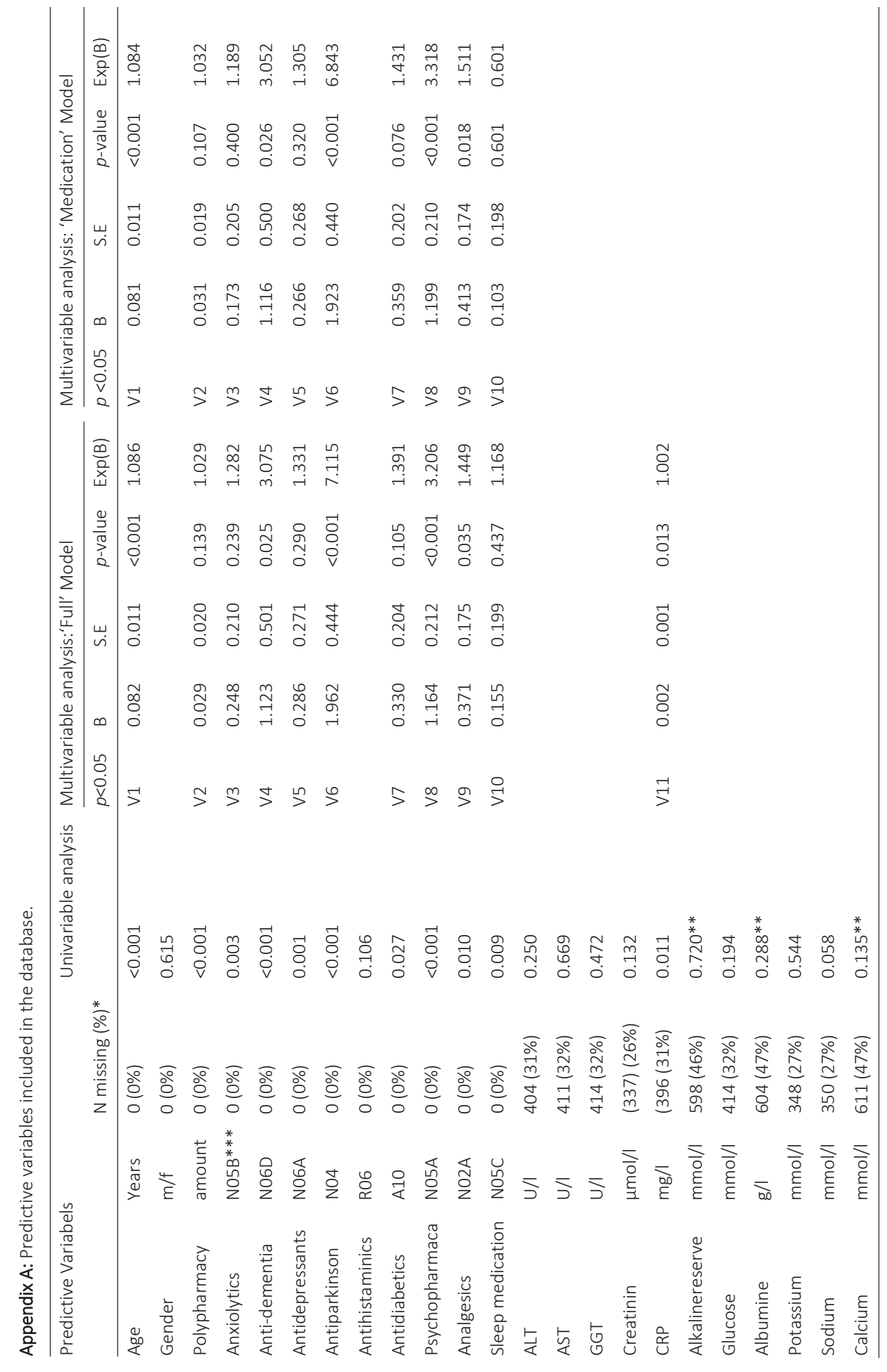


The development of an automated ward independent delirium risk prediction model

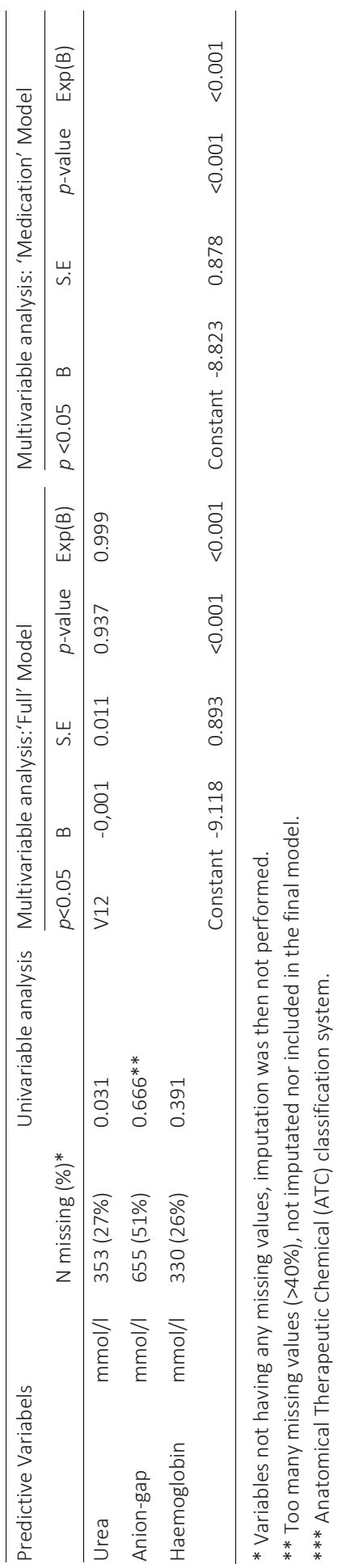





\section{Chapter}

General Discussion

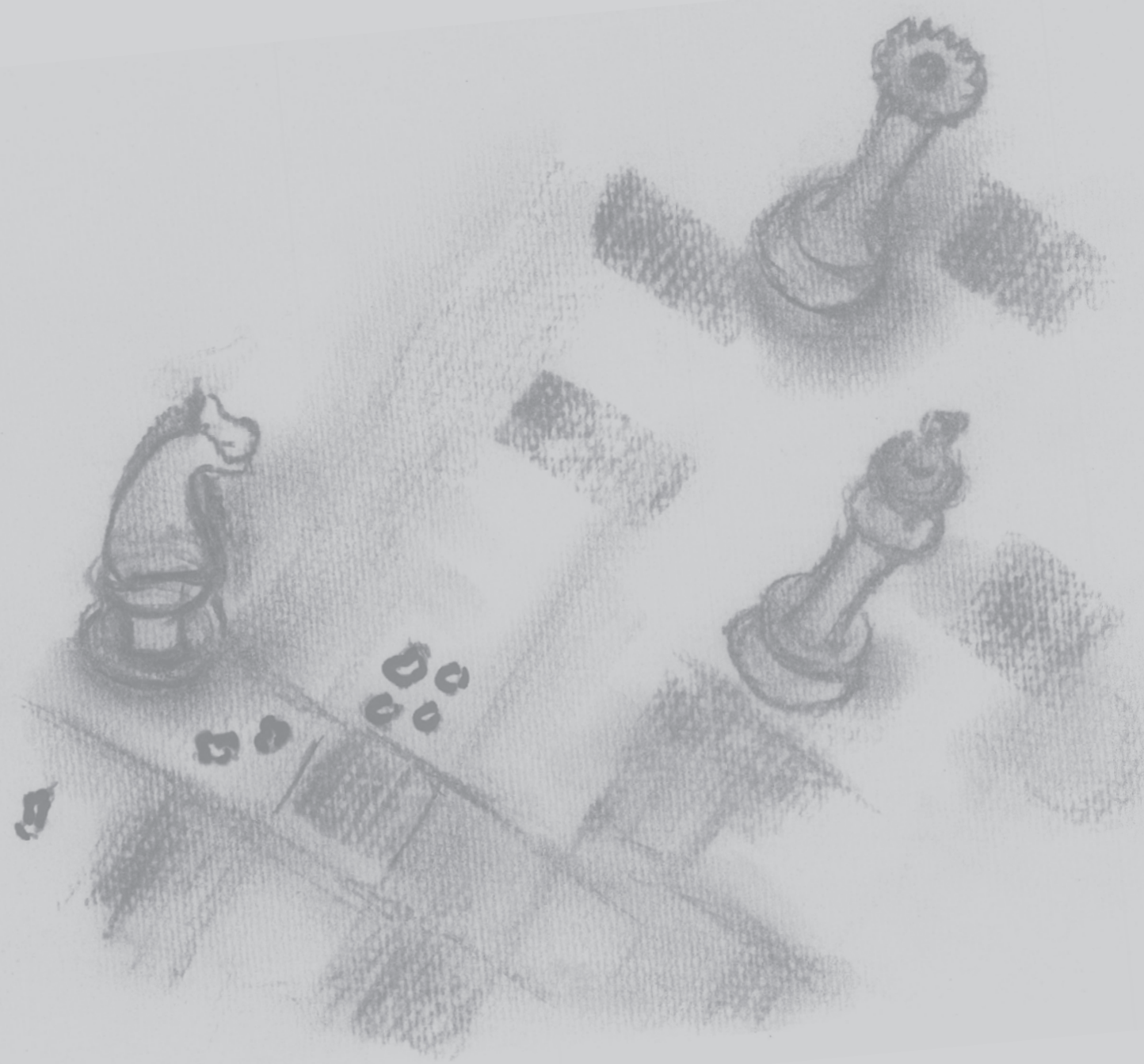



The use of medication increases with age (1). Polypharmacy, known as the concomitant use of many drugs, increases the likelihood of inappropriate prescription $(2,3)$, and has been shown to be an independent risk factor for hospitalisation. It is likely that half of these hospital admissions could have been prevented and were thereby a resultant of medication inappropriateness $(4,5)$. To tackle the problems of the increasing concomitant drug use and the increasing information from evidence-based data a clinical decision support system (CDSS) has become a feasible solution to automatically alert against pre-defined clinical problems. In addition to basic CDSS support for medication surveillance, e.g. drug-drug interactions, advanced CDSSs contain algorithms that combine multiple sources of information on individual patient characteristics such as the renal function combined with prescribed medication (6-8). The efficiency of advanced CDSSs, i.e. having a high percentage of clinically relevant alerts, is an important element for a successful implementation of CDSSs (9). Otherwise a CDSS that also presents many nonclinically relevant alerts would lead to alert fatigue. Alert fatigue is the ignoring of both the relevant and the not-relevant alerts (10). In addition to medication surveillance, the Dutch Healthcare Inspectorate (IGZ) expects a physician and a pharmacist to review the medication of all residents of nursing homes twice a year and once per year of the residents of residential homes, respectively, since these residents are prone to polypharmacy (11). A medication review is the process in which a health professional reviews the patient's drug treatment in relation to the diseases present, taking into account adverse drug effects, interactions, the necessity of the use of each drug, compliance, and the patient's understanding of the treatments thereby aiming to optimise the patient's pharmacological treatment (12). Literature reviews have shown that patients with polypharmacy will benefit from medication reviews; however outcomes such as mortality and hospitalisation remain to be debatable due to the fact that the various types of medication reviews, tools, and sample size are all important factors to take into account in the study of clinically relevant effects in studies (13-16). It seems reasonable to assume that when using non-automated reviewing tools, such a tool is only as good as the health care professional using it. Outcomes of performing medication reviews will depend on variables such as the reviewer's knowledge of evidence-based guidelines or perhaps the focus of the reviewer. Also, the reviewing tools that manually support medication reviews appear to be time-consuming, and these medication reviews only have temporary effects since they are performed infrequently. As soon as an advanced CDSS would be able to support medication reviews, it could provide surveillance around the clock, without any limitations to the amount of patients, and it would be consistent in the application of evidence-based data, i.e. finding the pre-defined medication inappropriateness.

Apart from medication surveillance and possibly medication reviews a CDSS might also be used for prediction models of subacute disease risk on an individual level in order to initiate timely (preventive) treatment $(17,18)$. Delirium is a disorder of cognition and attention, and a sign of the brain's vulnerability to external factors, which often 
occurs in frail elderly admitted to the hospital or residing in nursing homes (19). The automated prediction of delirium can be useful since it has been reported that many delirium diagnoses are missed in clinical practice (20). The development of such a model, exclusively using the available electronically risk factors, also appears useful when considering the availability of effective preventive (non-pharmacologic) treatment and the importance of the disease which is reflected by increased mortality, morbidity, costs, and its high incidence (19-24).

In the first study, described in chapter two, we wanted to find out the compliance of pharmacists with the requirements of the Dutch Healthcare Inspectorate (IGZ) by identifying the amount of currently performed medication reviews and the effort needed to perform the required medication reviews. This was the first objective of the thesis. We therefore conducted an explorative survey among pharmacists to reveal the clinical practice of medication reviews for older people in residential and nursing homes, in which we focussed on three important questions.

I To which extent are medication reviews performed by Dutch pharmacist as required by the Healthcare Inspectorate (IGZ)?

The IGZ expects all residents of nursing homes and residential homes to receive a medication review by a physician and a pharmacist twice a year and yearly, respectively (11). On the basis of the survey results, we could conclude that $42 \%$ to $76 \%$ of the required medication reviews are performed. This demonstrates that the medication reviews are not completely carried out according to the IGZ requirements. In the United Kingdom, the performance of medication reviews is included in the contractual requirements of general practitioners which demand a medication review for all patients being prescribed 'repeat' medication every 15 months. Furthermore, all National Health Services organisations are required to perform medication reviews in patients older than 75 years of age annually from 2002 onwards (25). However, we were unable to find any data on the compliance with these requirements.

Although the IGZ does not discuss selection of at risk patients in institutions, pharmacists may make a selection of at risk patients thereby excluding some of the residents of nursing homes and residential homes from medication reviews. Patient selection based on a minimum number of drugs is also regularly used in the inclusion criteria of studies, such as in the PHARM study (26). Clarification for the reasons behind the underperformance of the medication reviews required by the IGZ led to the next two questions investigated in the survey.

II What is the time needed to perform the required medication reviews?

III What is the economic efficiency and medical relevance of the medication reviews according to the pharmacist?

The survey results showed that the time needed to perform one medication review by a pharmacist was a mean of 29 minutes. It was calculated that it would thus yearly take a 
pharmacist 2.5 months full-time equivalents to perform the required medication reviews for an average-sized pharmacy (a mean of 731). This enormous time-effort to perform the medication reviews required by the IGZ seems to be an important factor why in practice this IGZ demand is not and cannot be fulfilled. Even more so, since a report from the Royal Dutch Pharmacists Association (KNMP) has determined that a single medication review for a patient in an institution, such as a nursing home, would require 68 minutes (27). If this data is applied to the reported mean number of patients per pharmacy from our survey, this would annually result in 6 months full-time work for a pharmacist which is more than twice the time reported in the survey. It is therefore safe to conclude that the calculated time needed to perform all the IGZ required medication reviews can hardly be achieved by a pharmacy.

Despite these results there is also awareness among pharmacists of the benefits of performing medication reviews. The pharmacists reported that they perceive medication reviews to be medically relevant, probably because such reviews might improve the quality of the pharmacotherapy. This result was to be expected from pharmacists despite the fact that the benefits on primary outcomes remain debatable. Nonetheless, a study on medication reviews in care homes showed a decrease of medication related problems and an increase of medication appropriateness (28). In another survey among physicians, general practitioners, and nursing home physicians, it was also concluded that the IGZ requirements were not met; with 'workload' being the most important limiting factor in the performance of medication reviews. However, all nursing home physicians reported the medication reviews to have a positive effect on the quality of care (29). This shows that medication reviews are considered to be of importance by both physicians and pharmacists, but it is difficult to actually carry out all the medication reviews.

Due to the explorative nature of the survey and its limitation to pharmacists, the results do not provide a complete view of the situation in the Netherlands, and should be interpreted cautiously in reference to the aspect of generalisation. A validated survey, among different types of healthcare professionals, would have been the optimal way to investigate the current state of the application of medication reviews in the Netherlands. The present survey also had a low response rate of $19 \%$ and, as our survey was anonymous, we were unfortunately unable to determine whether our survey sample was representative on the basis of specific respondents' characteristics or not. Nevertheless, the relatively large standard deviations of the respondents' characteristics suggest an acceptable heterogeneity in the group of respondents, together with the consistency in the respondents' answers, and we therefore believe that the information gathered was sufficiently reliable to answer the research questions. A solution for the time-demanding medication reviews in enormous groups of patients might be automation of a part of the process of medication reviews. Medication surveillance which also aims to optimise medication, was initially also carried out manually (30). A CDSS, used in 
medication surveillance could therefore be very suitable for the purpose of medication reviews if it efficiently alerts against medication inappropriateness.

Consequently, the second objective of this thesis was to identify and quantify the positive and negative properties of a home-grown, standalone, advanced CDSS with the intention to improve its efficiency. Our study into these properties was described in chapter three. An efficient advanced CDSS is a pre-requisite for using a CDSS in the support medication reviews. The alerts generated by the home-grown, standalone CDSS used in this study focussed on patients at risk, and took into account individual pharmacokinetics such as medication influenced by renal function, adverse side effects such as leucocytosis or hyperkalaemia as a result of medication, and specific dose related alerts such as weekly prescribed methotrexate. The home-grown CDSS showed to have limited efficiency with a mean of $3.6 \%$ of the alerts being clinically relevant in relation to all alerts presented by the CDSS. Only a few algorithms had an efficiency higher than $10 \%$. Two other studies also reported on the efficiency of a standalone CDSS. Rommers et al. reported an efficiency, i.e. intervention by the hospital pharmacist, of 3.7\% vs. $19.4 \%$ for the G-standard and the advanced CDSS, respectively (31). Eppenga et al. showed an increase of relevant drug safety alerts (5.8 to 17\%) when augmenting the G-standard with an advanced CDSS (7). This shows that the efficiency of the home-grown CDSS in our study was as low as that of the non-augmented G-standard. A high efficiency of any medication surveillance system is important, as a low efficiency has been shown to induce alert fatigue. It has already been reported that physicians ignore drug safety alerts in $49 \%$ to $96 \%$ of the cases (10). Increasing the efficiency of CDSSs to an efficiency level of more than $80 \%$ should be the first step to eliminate alert fatigue and to be able to efficiently support medication reviews with a CDSS.

For the improvement of the efficiency of an advanced CDSS three categories were defined that influence the efficiency: 1) algorithm alert criteria, 2) CDSS optimisation, and 3) data delivery. The first category, the algorithm alert criteria, should consider the dosage versus specified estimated Glomerular Filtration Rate-Modification of Diet in Renal Disease (eGFR-MDRD) boundaries. The second category, CDSS optimisation, addresses the notion that the CDSS should suppress repeat alerts after an initial signal has already been given, until a relevant change in one of the alert criteria makes the alert reappear. For the third category, data delivery, a direct link ( $\mathrm{HL} 7)$ from the laboratory system and the CPOE to the CDSS is important so that the patient's characteristics are always up-to-date. With these three defined categories we showed how the currently used advanced CDSSs can be improved with regard to their efficiency. The improvement of other defined requirements for the development of the CDSS were: 1) the simple adjustment or implementation of algorithm alert criteria, 2) validation of the CDSS, 3 ) easy implementation, 4) use of recognised technical standards for sharing decision support content, 5) easy to use in daily practice, and 6) the CDSS should provide information on previous alerts and actions. In another study, the success factors for an advanced CDSS were described as the "right message, right time, right place and right 
system" (32). These four categories are similar to our categories since the "right message" focuses on the sensitivity and specificity of the content, while the "right time" aims for integration of the CDSS in the workflow thereby saving time, which is described in our category as to whether it is "easy to use in daily practice". The "right place" is the delivery of alerts to the right professional, which is covered in our CDSS, since the alerts are aimed exclusively at physicians and pharmacists. The "right system" refers to the integration of a CDSS in other systems such as the electronic health record (EHR) or a computerised physician order entry system (CPOE). It is important to have a CDSS for medication optimisation integrated in other systems, because physicians can then receive an alert directly during the prescribing process. However, a CDSS integrated in an EHR or CPOE limits the possibility of easy implementation because integration in another system is complicated due to the large heterogeneity of other health systems (33). Therefore, a standalone CDSS currently remains the most commonly implemented CDSS for medication optimisation, but web-based solutions might lead to easier integrations. The above mentioned categories for success factors of advanced CDSSs match the requirements defined in our study and can be considered to be 'basic tools' for a successful implementation of a CDSS. The development and use of advanced CDSSs is a relatively new concept in healthcare and therefore these apparently 'basic tools' are not yet always integrated in the applied CDSSs. Our study undoubtedly showed which categories are important to increase the efficiency of the CDSS.

However, the fact that it was a retrospective study presents a limitation. A prospective study would have enabled us to investigate whether the physicians considered the alerts relevant or not, which would have helped us to even further optimise the alerts. Nonetheless, the current study does give insight into the aspects necessary for a highly efficient advanced CDSS. By taking the important aspects for an advanced CDSS into consideration we initiated the development of a CDSS that can be used to support medication reviews.

The development phases of the new CDSS have been described in chapter four. Phase 1 describes the CDSS design and shows that the CDSS is broadly applicable in nursing homes and other healthcare settings such as hospitals. Phases 2 and 3 discuss the development of the algorithm content and the validation of the CDSS, respectively. A randomised controlled trial to show the possible benefits of the CDSS in nursing homes is described in phase 4, while the implementation of the system in other nursing homes in order to determine feasibility for the implementation of the CDSS in another setting is discussed in phase 5 . These five phases are based on the Systems Development Life Cycle (SDLC). Different approaches of the SDLC, are possible; our approach resembles the 'iterative prototyping', which describes the iterative process of defining the requirements, designing the system, coding the system, and testing the system, after which the system is implemented. This method is specifically appropriate when evolving from prototype to working system with a good likelihood of users acceptance 
(34). The phases described are applied in such a way that they optimally suited the development and implementation of the CDSS.

The idea to automate medication reviews was not new, but our approach which does not require the manual input of patients' characteristics to perform medication reviews, was to the best of our knowledge described for the very first time.

The use of a semi-automatic CDSS to support medication reviews has been described earlier in this thesis. This CDSS was based on a computer programming technique known as knowledge acquisition; a subfield of artificial intelligence. This method allows individualisation of the alerts by 'automatically' learning from the user defined relevant alerts (35-37). A study that used the CDSS based on knowledge acquisition, and referred to the commercial system 'Medscope Review Mentor', claimed that the data could also be directly imported from physician software. However, in this study the patients' characteristics were entered into the system manually which required 10 minutes for each patient (38). The CDSS desgined by us works exclusively by using the time-efficient automated input of patients' characteristics, which will be needed to optimally perform all the medication reviews required by the IGZ. As mentioned earlier, the CDSS was developed on the basis of the experience and analyses from the previously home-grown standalone advanced CDSS. However, it has been assumed that the support of medication reviews by using a CDSS would decrease the time needed to perform the complete medication review process, whereas a recent study showed that the time needed to perform medication reviews, also using a semi-automatic CDSS such as the 'Medscope Review Mentor', increased (39). According to the authors increase in time and decrease of efficiency could be attributed to unfamiliarity with the new method and the user-interface of the CDSS. Indeed, the authors have more recently shown a significant decrease in the time needed when experience had been gained in the method used (40). We believe that the automated input of patients' characteristics into a CDSS and automated output of alerts will be the optimal way to efficiently support medication reviews. We therefore aimed to develop a knowledge-based CDSS that contains algorithms that automatically alert against pre-defined problems by using decision tree rules without having to enter patient characteristics manually, despite the fact that knowledge-based CDSSs with pre-defined decision tree rules are known for their limited implementation due to the great effort required to create and maintain these systems (33). Another method, the use of a 'knowledge acquisition' CDSS that also supports medication reviews, was described earlier in this thesis (see addendum of chapter 4). This method contains self-learning software using 'multiple classification ripple down rules' (MCRDR), which enables the user to define the decision tree rules in the system (37). Although self-learning software is apparently a useful technique, we regard a CDSS with pre-defined decision tree rules as having clear benefits when compared to self-learning software. It is questionable whether a system in which a professional creates an individualised system by using self-learning software, would produce all the alerts needed. A study has shown that physicians and pharmacist only identified 
approximately one-third of the problems that an expert-group would detected, which confirms that healthcare professionals can have 'blind spots' for drug-related problems (41). By using a system only based on the 'automatically' learning from the user defined relevant alerts, as defined by the user, it might be possible that the healthcare professional unintentionally introduces a low specificity level. In our opinion the use of a validated CDSS with pre-defined problems, based on consensus, will therefore detect more drug-related problems than a CDSS based on 'knowledge acquisition'. However, using a technique of 'knowledge acquisition' in combination with the review of these automatically learned algorithms by an expert team could limit the efforts of maintaining a CDSS.

The third objective of this thesis was to explore the possibilities of supporting medication reviews with the developed advanced CDSS thereby focussing on the interventions found in medication reviews. The study described in chapter five is a proof of concept study to demonstrate the feasibility of the CDSS to support medication reviews, and also to uncover its strengths and weaknesses to further improve the system.

In chapter five, the developed advanced CDSS was compared with manually performed medication reviews of hospitalised patients. During the manual medication reviews, a mean of 7.0 (SD 2.2, range 2-11) drug-related problems (DRP strategies) were found which shows that conventional medication reviews in hospitalised older adults can be highly effective for the identifications of DRPs. In a systematic review of the literature concerning medication reviews among hospitalised patients (age ranging from 5-87 years) the amount of drug-related problems per patient varied from 0.13 to 10.6 (42). Since we have studied a geriatric population, it seems plausible that we found a relatively high amount of DRPs per patient. Although we did not study patient outcomes, other studies reported positive effects of medication reviews in hospitalised patients such as: positive outcomes on re-admission, drugs related re-admission, increase of the quality of prescribing, and a relative risk reduction in the number of emergency department contacts $(15,42)$.

In total 249 clinically relevant DRPs were determined, out of which the CDSS had alerted against 70 DRPs. These DRPs were confirmed as relevant in the multidisciplinary gerontopharmacology meeting or by the authors when considering CDSS alerts that had been previously unidentified in the medication reviews. Of these CDSS alerts, 63\% (44) were also found in the conventional medication reviews and $10 \%$ (26) were undiscovered DRPs. There are also studies showing the effects of a CDSS on medication prescriptions $(43,44)$. Moreover, Thier et al. have shown that the adherence to guidelines in clinical practice is rather low for many chronic diseases (45), while a CDSS has already proven to serve as a good instrument to support adherence to guidelines $(31,39,46)$. However, to our knowledge there is only limited amount of studies that compare the use of an automated advanced CDSS with a conventional medication review. One study which used the knowledge acquisition CDSS known as 'Medscope Review Mentor', showed supremacy of the CDSS compared to the pharmacists in the performance of 570 medication reviews. The CDSS found significantly more DRPs than the 
pharmacists, with 129 different types of alerts (themes) (38). In this study, the CDSS alerts are merely compared with the findings of the pharmacist, but without challenging the DRPs with physicians in a medication review. A study from the Netherlands also suggests that a CDSS may improve the effectiveness of medication reviews. The researchers aim to integrate the CDSS into a CPOE, but in the study the manually entering of patient characteristics was still required to initiate alerts (39). Taking into account these studies, and our own study, we believe that a CDSS is able to support medication reviews. It appears, however, that the full potential of using a fully automated advanced CDSS to alert against drug-related problems normally identified in manually performed medication reviews, has not been shown yet.

In the development of the CDSS, a high efficiency level was aimed for to prevent alert fatigue. In the study, described in chapter five, the sensitivity and specificity of the alerts reported by the CDSS was $72.9 \%$ and $98.6 \%$, respectively. However, many alerts (179 out of 249) were not detected by the CDSS, which urged us to improve the relatively poor performance of the CDSS in the support of medication reviews. We therefore identified its limitations and strengths on the level of clinical relevance of the drugrelated problems. The classification of types of medication errors not found in the conventional medication reviews has shown that the CDSS is good in retrieving medication error types concerning 'contraindications/interactions/side effects' and 'indication without medication'. These medication errors types are similar to a subset of errors found in the evaluation of the causes of preventable medication related hospital admissions $(5,47)$. This suggests that the CDSS is able to find important medication errors that, if correctly intervened, might prevent medication related hospital admissions. Yet the medication errors types mostly not found by the CDSS were 'medication without indication', 'double medication', and 'wrong medication'. The lack of detecting these medication error types by the CDSS is multifactorial because of the complexity of these errors and the fact that the manually performed medication reviews were compared with the advanced CDSS, but did not take into account the regular care which also includes medication surveillance from the CPOE integrated medicines databank. The defined 'double medication' errors may result from alert fatigue of the relatively sparse clinically relevant CPOE alerts generated by the 'G-standard. The lack of finding other medication error types results from the lack of input of documented multi-morbidity from electronic health records, the under-development of deprescribing algorithms, and the under-development of indicators that would enable a CDSS to interpret the progression or status of indications and adverse drug events.

The study was performed in a limited number of hospitalised patients without measurement of patient-outcomes and, therefore benefits of using a CDSS in the support of medication reviews should not be generalised. Advanced CDSSs are at this time an emerging technology and were relatively recently introduced into healthcare which makes the described aspects especially important to consider in further improvements of CDSSs. 
A multi-centre study in nursing homes, using the CDSS from this study, is currently being carried out and aims to show patient-outcomes as well (48).

\section{Improvement and addition of new clinical rules}

In order to be able to provide a better support for medication reviews the CDSS algorithms need to be complemented by adding the unnoticed DRPs from the conventional medication reviews and by optimizing the DRPs that were incorrectly assessed by the CDSS. These improvements can be introduced by simply adding information to the existing algorithms, such as an exclusion trigger. For example, an exclusion trigger to the algorithm that alerts against an elevated INR related to an anticoagulant overdosage, could be the prescription of vitamin K. However, some suggestions might be more complex such as the addition of an indicator for bowel movements (i.e. stool) to be able to differentiate between patients that require laxative prescription or not. Currently the advice for a laxative is based on the prescription of an opiate without incorporating clinical symptoms. The inclusion of criteria for clinical symptoms in algorithms (clinical criteria) can be beneficial to increase the alert sensitivity, i.e. more clinically relevant alerts. For example, alerts for patients that would require a laxative according to guidelines, but are suffering from diarrhoea would not occur. If such indicators are documented in the electronic health records, these should be made available for automation purposes and a (validated) cut-off point to trigger such an algorithm should be developed. From this thesis can be concluded:

Advice 1: clinical criteria (i.e. indicators) that will improve the efficiency of alerts should be developed.

\section{When to perform medication reviews}

The Dutch government has on purpose introduced a hierarchy in the healthcare system which aims to lead to minimal claims on the highest (i.e. most expensive) step: admission to nursing homes and residential homes. Hospitalisation might be perceived as a much more serious event than the admission into an institution such as a nursing home, but on a population level the mean usage of long-term care of elderly people (age $>65$ years) in institutions is more expensive than short-term hospitalisation (49). It seems strange that during hospitalisation medication reviews are not obliged by the IGZ nor by the health insurance companies, while reviews are proven to have positive outcomes in hospitalised patients $(15,42)$. In chapter five we have found a mean of 7 drug-related problems as an outcome of performing medication reviews in hospitalised patients. More than half of these drug-related problems were related to 'indication without medication' (e.g. atrial fibrillation without anticoagulants) and 'medication without indication' (e.g. selective 


\section{Chapter 7}

alpha 1-adrenoceptor antagonist usage after transurethral resection of the prostate or the use of gastrointestinal prophylaxis in patients despite the fact that the use of the risk induced drug had already been discontinued). Although community-dwelling elderly people would also receive medication reviews after hospital discharge, these would most likely be carried out much later than the discharge. Furthermore, patients are already questioned about their medication use when they are admitted and discharged from the hospital. This is defined as medication reconciliation which aims at assembling an accurate medication list that includes documentation of changes made to the medication. The correct transfer of medication use is important to minimise the risks of adverse drugs events, early re-admissions after discharge, and prolonged hospitalisation (50). The moment of medication reconciliation is perhaps the perfect moment to also discuss medication agreement as intended in (clinical) medication reviews. The input of the patient's information, such as drug-adherence or symptoms of adverse drug events (51), in combination with a multidisciplinary approach currently has not yet been automated. Therefore the following advice should be considered:

Advice 2: medication reviews should also be structurally performed in hospitalised patients next to patients in nursing homes, residential homes, and community dwelling elderly.

\section{Automation of medication reviews}

In this thesis we have concluded that the currently required medication reviews are very time-consuming to perform and thus very costly. It is therefore imperative that more research and progress is made in automation of medication reviews. Additionally, it was also reported that different types of physicians in the hospital setting, i.e. medical specialists, have a different focus on the medication they prescribe (52). Training to perform quality medication reviews could be a solution (53). However, even experienced physicians and pharmacists have shown a low efficiency in manually performed medication reviews when compared to an expert group (54). To be able to perform the required and demanded medication reviews an efficiency enhancement would be very valuable. Therefore, further research and efforts should be initiated to automate the medication review process as much as possible, with the common objective to increase the efficacy of automatically alerting against all drug-related problems. The most obvious start is to document patients' characteristics so these will be readily available for automation, such as 'indications for medications'. On the basis of this, we give the following advice:

Advice 3: data delivery of patients' characteristics should be easily available for automation since these are needed to automate medication reviews. 
The fourth objective of this thesis was to explore the possibilities of using a CDSS for the prediction of an important sub-acute illness in hospitalised patients, i.e. developing a delirium predictive algorithm in hospitalised older adults. Such an algorithm would allow to automatically calculate the individual risk of these patients in order to start timely preventive treatment. The study described in chapter six is a proof of concept study to demonstrate the feasibility of the hospital systems to predict the risk of a delirium automatically, by exclusively using the currently available electronic risk factors. In this study we have described the development of a ward independent delirium algorithm that automatically predicts the individual risk of a delirium in hospitalised patients, by exclusively using electronically available documented patient characteristics. With regard to the patient characteristics used, these are presumed to be easily available in any hospital for the use in a CDSS without having to adjust hospital software. The developed model can be regarded as clinically relevant, taking into account the sensitivity of $78.2 \%$, specificity of $63.7 \%$, and AUROC of 0.76 , and can be helpful to start timely preventive delirium treatment for at-risk patients. Recently an association was also found between high-risk and very-high-risk delirium patients and an increased risk for readmission, length of stay, and discharge to rehabilitation. Early detection of patients with a high risk or a very high risk to develop delirium could be very beneficial (55). Delirium predictive models already exist for hospitalised elderly patients and intensive care patients, but these models rely on the manual calculation of the patient's individual risk (56-59). Kobayashi et al. have also developed a delirium risk prediction model by retrieving the patients' information from the electronic charts, but this was merely done for internal medicine units (60). In contrast, our model has been developed to automatically predict a delirium by exclusively using electronically available risk factors and can be used throughout the hospital.

Furthermore, in the study by Kobayashi a chi-square automatic interaction detector (CHAID) decision tree model is proposed instead of a logistic model for delirium risk prediction because the AUROC of the CHAID decision tree model was higher than that of the 'logistic model' (0.82 vs. 0.79) suggesting that a decision tree analysis method is more sensitive and specific. A decision tree model stratifies the individual patient risk, while a logistic model gives a continuous individual risk (60). A continuous risk will be more precise for the individual patients, while the mentioned disadvantage of the manual calculation in a logistic model is overcome if using a CDSS. In our opinion a CDSS that automatically calculates a patient's individual risk, will make the use of a stratification decision tree model redundant. A CDSS can combine the predictive variables and provide the individual risk scores through automation. Simplifying models to be of practical use is unnecessary when a CDSS is used and predictive variables are electronically available. Recently another study also developed an automated delirium prediction algorithm based on patients' characteristics from the electronic health record (EHR). This study was able to include the pre-determined risk factors: "impaired baseline cognition, vision impairment, severity of illness, fracture, infection, and age" by developing specific 
software to extract these characteristics from the EHR. The documentation of the risk factors that assess the cognitive function was determined as very time consuming (20 minutes), which was resolved by using brief cognitive assessments (55). We believe that if these predictive variables, and the other proven relevant predictive variables, including cognitive disorders, a high blood urea nitrogen /creatinine ratio, lower functional status, 'diagnosis group' (e.g. surgical vs. neurology patients), and 'urgent admission', would all have been available electronically, the predictive value would ultimately have been optimal $(56-58,61)$. The absence of these predictive variables in our model shows the lack of proper computerisation in the hospital, a computerisation which is highly necessary to develop the digital healthcare risk models of the future. Despite the awareness of the absence of some of the risk factors that were proven relevant, the development of an automated predictive delirium algorithm limited to the available electronically risk factors appeared useful, since this also revealed important aspects necessary for further developments.

The study described is a retrospective cohort that used an internal validation method. It should, however, be noted that the prediction quality of the model may be an overestimation of the true prediction quality, as this was tested on the same dataset on which the model had been developed. In addition, testing it on the same dataset does not deal with selection-bias. The model should therefore be externally validated, e.g. in another institution, such as another hospital or a nursing home, which consists of a different population where the delirium incidence might differ from the one in the cohort used in our retrospective study.

\section{Predictive medicine with a CDSS}

Further automation and concomitantly the development of much more accurate predictive models, relies on the 'level' of computerisation of databases. Computerisation suggests that the patients' characteristics necessary are digital, but automation is less evident if these patients' characteristics are simply a digital version of a printed document $(62,63)$. Predictive medicine is optimally developed by using large patient databases and is sometimes also referred to as 'the analysis of big data'. Big data is the enormous amount of complex and linked data from multiple sources. Exponential progress in computer calculating power makes it possible to find associations in big data that regular database systems are unable to find (64). Supercomputers, containing a plethora of algorithms, are able to 'read' the digital version of printed documents. Nevertheless, the reading of such texts introduces new problems, for instance the understanding of a language or knowing the medical taxonomy in a small language such as the Dutch language by a supercomputer (65). It seems that especially in the Netherlands, we should pay more attention to documentation for the purpose of automation. Although physicians are well aware of the importance of documentation, endless documentation has been shown to occupy up to half of the physician's time and is hard to 
combine with clinical work (66). Also, risk factors derived from patient observations, such as a cognition screening method, are known to be time-consuming (55). Documentation of adverse events, of patients reported outcomes, and of patients' characteristics will be essential to allow the development of CDSSs able to optimally support medication reviews. In the absence of proper automated documentation of patient characteristics for physicians and nurses, the documentation will remain limited and the full support of clinical medication reviews by a CDSS will not be possible. A good solution is therefore needed for the automation of documentation, since the time needed for documentation will increase and will thus remain a stressor on clinical work.

Advice 4: data delivery of patients' characteristics should be easily available for automation since these are needed to automate predictive algorithms.

\section{Implications for the use of a CDSS to optimise treatment of patients}

One specific aspect connects the different chapters of this thesis, and this is the use of an efficient CDSS for variable clinical problems: 1) advanced medication surveillance, 2) medication reviews, 3) predictive medicine in subacute illnesses. As described before, the advanced medication surveillance should be optimally integrated into the CPOE, especially since the alerts from advanced medication surveillance augment the alerts from basic medication surveillance $(6,7)$. However, whether the alerts related to medication reviews should also be integrated into the CPOE can be a matter for debate, since a medical specialist in the hospital will most probably regard alerts related to medication reviews as irrelevant for his profession. For example, it is unlikely that an otolaryngologist would feel responsible for the revision of the use of a statin when he or she is prescribing ear drops. Taking this into consideration, there should perhaps be a clear difference between acute drug safety alerts and drug safety alerts for the longer term. Yet an advanced CDSS integrated into CPOEs can give feedback during prescription process, thereby preventing DRPs on a population level rather than having infrequently performed medication reviews to repair overdue maintenance (67). Furthermore, the use of a CDSS in the prediction of a subacute illness, such as a delirium, should not be presented in the CPOE but rather in the EHR. This might also be the case for some medication reviews alerts. It is clear that drug safety alerts or risk assessments should be presented 'at the right time, place, and in the right system' (9), but it is not clear how this should be achieved when the same standalone CDSS is used in different workflows. Currently CPOEs are often not yet integrated into the EHR: however, all these different hospital systems will slowly evolve into one integrated system (68). An optimal approach would be a 'cockpit' integration of the CDSS into the EHR that would allow for tuning of alert presentation in different workflows, for instance, the prescription of medication requires advanced medication surveillance, and a patient admitted 


\section{Chapter 7}

into hospital will need a delirium risk prediction as a part of the general overview of the patient (18). Advanced CDSSs for medication surveillance are often primarily screened by pharmacists or trained employees after which physicians are approached to approve the suggested interventions (7, 31). Another approach could be to send an e-mail to the appropriate physician containing an Alert-URL allowing the physician to open the alerts in a web browser without requiring direct access to a CDSS or without the CDSS having to be integrated into the hospital systems. This is, however, a work-around that can be considered as awaiting more optimal solutions. These suggestions are also important for the successful implementation of CDSSs aimed at medication optimisation. Overall, CDSSs are a relatively new technique used and this thesis has explored its use for variable clinical problems. 


\section{REFERENCES}

1. Lemmens LC, Weda M. Polypharmacie bij kwestbare ouderen: inventarisatie van risico's en mogelijke interventiestrategieen [Internet]. 2013 RIVM. [ cited on 20 nov 2015] Available from: http://www.rivm.nl/ Documenten_en_publicaties/Wetenschappelijk/Rapporten/2013/juli/Polyfarmacie_bij_kwetsbare_ouder en_Inventarisatie_van_risico_s_en_mogelijke_interventiestrategie\%C3\%ABn.

2. Gallagher PF, Barry PJ, Ryan C, Hartigan I, O'Mahony D. Inappropriate prescribing in an acutely ill population of elderly patients as determined by Beers' Criteria. Age Ageing. 2008;37(1):96-101.

3. Cherubini A, Corsonello A, Lattanzio F. Underprescription of Beneficial Medicines in Older People: Causes, Consequences and Prevention. Drugs Aging. 2012;29(6):463-75.

4. Pedrós C, Quintana B, Rebolledo M, Porta N, Vallano A, Arnau J. Prevalence, risk factors and main features of adverse drug reactions leading to hospital admission. Eur J Clin Pharmacol. 2014;70(3):361-7.

5. Leendertse AJ, Egberts AC, Stoker $L$, van den Bemt PM, Group HS. Frequency of and risk factors for preventable medication-related hospital admissions in the Netherlands. Arch Intern Med. 2008;168(17):1890-6.

6. Helmons PJ, Suijkerbuijk BO, Nannan Panday PV, Kosterink JGW. Doing the right things and doing things right: inpatient drug surveillance assisted by clinical decision support. Eur J Hosp Pharm. 2015;22(4):236-42.

7. Eppenga WL, Derijks HJ, Conemans JM, Hermens WA, Wensing M, De Smet PA. Comparison of a basic and an advanced pharmacotherapy-related clinical decision support system in a hospital care setting in the Netherlands. J Am Med Inform Assoc. 2012;19(1):66-71.

8. Tawadrous D, Shariff SZ, Haynes RB, lansavichus AV, Jain AK, Garg AX. Use of clinical decision support systems for kidney-related drug prescribing: a systematic review. Am J Kidney Dis. 2011;58(6):903-14.

9. Scheepers-Hoeks AM, Grouls RJ, Neef C, Korsten HH. Strategy for implementation and first results of advanced clinical decision support in hospital pharmacy practice. Stud Health Technol Inform. 2009;148:142-8.

10. van der Sijs H, Aarts J, Vulto A, Berg M. Overriding of drug safety alerts in computerized physician order entry. J Am Med Inform Assoc. 2006;13(2):138-47.

11. Dutch Healthcare Inspectorate. Beoordelingskader medicatieveiligheid verpleeghuizen. [ cited on 20-012016]. Available from: http://www.igz.nl/Images/Bijlagen\%20Beoordelingskaders\%20en\%20Risicoscore \%20bij\%20rapport\%20Medicatieveiligheid_tcm294-287277.pdf.

12. Zermansky A, Petty D, Raynor T, Lowe C, Freemantle N. Clinical medication review by a pharmacist of patients on repeat prescriptions in general practice: a randomised controlled trial. Health Technol Assess. 2002;6(20):86.

13. Topinkova E, Baeyens JP, Michel JP, Lang PO. Evidence-based strategies for the optimization of pharmacotherapy in older people. Drugs Aging. 2012;29(6):477-94.

14. Hatah E, Braund R, Tordoff J, Duffull SB. A systematic review and meta-analysis of pharmacist-led fee-forservices medication review. Br J Clin Pharmacol. 2014;77(1):102-15.

15. Christensen $M$, Lundh $A$. Medication review in hospitalised patients to reduce morbidity and mortality. Cochrane Database Syst Rev. 2013;2:CD008986.

16. Wallerstedt SM, Kindblom JM, Nylen K, Samuelsson O, Strandell A. Medication reviews for nursing home residents to reduce mortality and hospitalization: systematic review and meta-analysis. $\mathrm{Br} \mathrm{J} \mathrm{Clin}$ Pharmacol. 2014;78(3):488-97.

17. Oh SM, Stefani KM, Kim HC. Development and application of chronic disease risk prediction models. Yonsei Med J. 2014;55(4):853-60.

18. Stusser R, Dickey R. Quality and Cost Improvement of Healthcare via Complementary Measurement and Diagnosis of Patient General Health Outcome Using Electronic Health Record Data: Research Rationale and Design. J Med Syst. 2013;37(6):1-13.

19. Inouye SK, Westendorp RG, Saczynski JS. Delirium in elderly people. Lancet. 2014;383(9920):911-22.

20. Siddiqi N, House AO, Holmes JD. Occurrence and outcome of delirium in medical in-patients: a systematic literature review. Age Ageing. 2006;35(4):350-64. 


\section{Chapter 7}

21. Hshieh T, Yue J, Oh E, Puelle M, Dowal S, Travison T et al. Effectiveness of multicomponent nonpharmacological delirium interventions: a meta-analysis. JAMA internal medicine. 2015;175(4):512-20.

22. Neufeld KJ, Yue J, Robinson TN, Inouye SK, Needham DM. Antipsychotic Medication for Prevention and Treatment of Delirium in Hospitalized Adults: A Systematic Review and Meta-Analysis. J Am Geriatr Soc. 2016;64(4):705-14.

23. Schrijver EJ, de Graaf K, de Vries OJ, Maier AB, Nanayakkara PW. Efficacy and safety of haloperidol for inhospital delirium prevention and treatment: A systematic review of current evidence. Eur J Intern Med. 2016;27:14-23.

24. Leslie DL, Marcantonio ER, Zhang Y, Leo-Summers L, Inouye SK. One-year health care costs associated with delirium in the elderly population. Arch Intern Med. 2008;168(1):27-32.

25. NHS Cumbria Medicines Management Team. Clinical Medication Review: A practice guide. 2013. [ cited on 27 Jan 2016]. Availble from: www.cumbria.nhs.uk.

26. Leendertse AJ, de Koning FH, Goudswaard AN, Jonkhoff AR, van den Bogert SC, de Gier HJ et al. Preventing hospital admissions by reviewing medication (PHARM) in primary care: design of the cluster randomised, controlled, multi-centre PHARM-study. BMC Health Serv Res. 2011;11:4.

27. Significant. Generieke kosten medicatiebeoordeling [Internet]. 2014.[cited on 20 Jan. 2016] Available from: http://www.knmp.nl/downloads/nieuws/downloads-nieuws-2014/SignificantRapportGeneriekekos tenmedicatiebeoordelingDefinitief.pdf.

28. Alldred DP, Raynor DK, Hughes C, Barber N, Chen TF, Spoor P. Interventions to optimise prescribing for older people in care homes. Cochrane Database Syst Rev. 2013;2:CD009095.

29. Hurkens KPGM, Mestres Gonzalvo C, de Wit HAJM, van der Kuy P-HM, Janknegt R, Verhey FR et al. A Survey on Medication Reviews in Older Patients: Substantial Variation in Daily Practice. J Gerontol Geriat Res 2013;2(133).

30. Winters DJ. KNMP-bestand: nieuwe basis voor Informatorium and Taxen. Pharm Weekbl. 1979(114):353-8.

31. Rommers MK, Teepe-Twiss IM, Guchelaar HJ. A computerized adverse drug event alerting system using clinical rules: a retrospective and prospective comparison with conventional medication surveillance in the Netherlands. Drug Saf. 2011;34(3):233-42.

32. Scheepers-Hoeks AMJW, Neef C, Ackerman EW, Korsten EHM. Efficient decision support systems practice and challenges in biomedical related domain [part of a book]. InTech; 2011.

33. Achour SL, Dojat M, Rieux C, Bierling P, Lepage E. A UMLS-based Knowledge Acquisition Tool for Rulebased Clinical Decision Support System Development. J Am Med Inform Assoc. 2001;8(4):351-60.

34. Centers for Medicare and Medicaid Services. Selecting a development approach [Internet]. 2008. [cited on 27 Jan. 2016]. Available from: https://www.cms.gov/Research-Statistics-Data-and-Systems/CMSInformation-Technology/XLC/Downloads/SelectingDevelopmentApproach.pdf.

35. de Wit HA, Mestres Gonzalvo C, Janknegt R, Schols JM, van der Kuy PH. A fully automated medication review? Int J Clin Pharm. 2014;36(2):220-1.

36. Bindoff IK, Tenni PC, Peterson GM, Kang BH, Jackson SL. Development of an intelligent decision support system for medication review. J Clin Pharm Ther. 2007;32(1):81-8.

37. Bindoff I KB, Ling T, Tenni P, Peterson G. Applying MCRDR to a multidisciplinary domain. Proceedings of the 20th Australian Joint Conference on Artificial Intelligence,Gold Coast, Australia: Springer. 2007. p. 519-28.

38. Curtain C, Bindoff I, Westbury J, Peterson G. An investigation into drug-related problems identifiable by commercial medication review software. Australas Med J. 2013;6(4):183-8.

39. Meulendijk MC, Spruit MR, Drenth-van Maanen AC, Numans ME, Brinkkemper S, Jansen PA et al. Computerized Decision Support Improves Medication Review Effectiveness: An Experiment Evaluating the STRIP Assistant's Usability. Drugs Aging. 2015;32(6):495-503.

40. Meulendijk MC, Spruit MR, Willeboordse F, Numans ME, Brinkkemper S, Knol W et al. Efficiency of Clinical Decision Support Systems Improves with Experience. J Med Syst. 2016;40(4):76.

41. Mestres Gonzalvo C, Hurkens KP, de Wit HA, van Oijen BP, Janknegt R, Schols JM et al. To what extent is clinical and laboratory information used to perform medication reviews in the nursing home setting? the CLEAR study. Ther Clinical Risk Manag. 2015;11:767-77. 
42. Graabaek T, Kjeldsen LJ. Medication reviews by clinical pharmacists at hospitals lead to improved patient outcomes: a systematic review. Basic Clin Pharmacol Toxicol. 2013;112(6):359-73.

43. Yourman L, Concato J, Agostini JV. Use of computer decision support interventions to improve medication prescribing in older adults: A systematic review. Am J Geriatric Pharmacother. 2008;6(2):119-29.

44. Marasinghe KM. Computerised clinical decision support systems to improve medication safety in longterm care homes: a systematic review. BMJ open. 2015;5(5):e006539.

45. Thier SL, Yu-Isenberg KS, Leas BF, Cantrell CR, DeBussey S, Goldfarb NI et al. In chronic disease, nationwide data show poor adherence by patients to medication and by physicians to guidelines. Manag Care. 2008;17(2):48-52, 5-7.

46. O'Sullivan D, O'Mahony D, O'Connor MN, Gallagher P, Cullinan S, O'Sullivan R et al. The impact of a structured pharmacist intervention on the appropriateness of prescribing in older hospitalized patients. Drugs Aging. 2014;31(6):471-81.

47. Howard R, Avery A, Howard P, Partridge M. Investigation into the reasons for preventable drug related admissions to a medical admissions unit: observational study. Quality Saf Health Care. 2003;12(4):280-5.

48. Mestres Gonzalvo C, de Wit HAJM, Hurkens KPGM, van Oijen BPC, Janknegt R, Schols JMGA et al. SCREAM: Supporting Clinical Rules in the Evaluation and Adjustment of Medication. Submitted.2016.

49. C. van Campen MBvG, D. Deeg, J. Ledema. Met zorg ouder worden. Zorg trajecten van ouderen in tien jaar [Internet]. 2013.[ cited on 20 Nov 2015]. Available from: http://www.scp.nl/Publicaties/Alle_ publicaties/Publicaties_2013/Met_zorg_ouder_worden.

50. Ensing HT, Stuijt CC, van den Bemt BJ, van Dooren AA, Karapinar-Carkit F, Koster ES et al. Identifying the Optimal Role for Pharmacists in Care Transitions: A Systematic Review. J Managed Care \& Spec Parm. 2015;21(8):614-36.

51. Praktijkperikel. Het protocol (een waar gebeurd sprookje). Medisch Contact. 2015;70(39).

52. Hurkens KPGM, de Wit HAJM, Mestres Gonzalvo C, Janknegt R, Verhey F, Schols, JMGA, Magdelijns F, et al. Optimizing a computer assisted medication review in hospitalized patients: a pilot study. Submitted. 2016.

53. Kwint HF, Faber A, Gussekloo J, Bouvy ML. Completeness of medication reviews provided by community pharmacists. J Clin Pharm Ther. 2014;39(3):248-52.

54. Mestres Gonzalvo C, Hurkens KPGM, de Wit HAJM, van Oijen BPC, Janknegt R, Schols JMGA et al. To what extent is clinical and laboratory information used to perform medication reviews in the nursing home setting? the CLEAR study. Ther Clinical Risk Manag. 2015;11:767-77.

55. Rudolph JL, Doherty K, Kelly B, Driver JA, Archambault E. Validation of a Delirium Risk Assessment Using Electronic Medical Record Information. J Am Med Dir Assoc. 2016;17(3):244-8.

56. van den Boogaard M, Pickkers P, Slooter AJ, Kuiper MA, Spronk PE, van der Voort PH et al. Development and validation of PRE-DELIRIC (PREdiction of DELIRium in ICu patients) delirium prediction model for intensive care patients: observational multicentre study. BMJ. 2012;344:e420.

57. Inouye SK, Viscoli CM, Horwitz RI, Hurst LD, Tinetti ME. A predictive model for delirium in hospitalized elderly medical patients based on admission characteristics. Ann Intern Med. 1993;119(6):474-81.

58. Carrasco MP, Villarroel L, Andrade M, Calderon J, Gonzalez M. Development and validation of a delirium predictive score in older people. Age Ageing. 2014;43(3):346-51.

59. Douglas VC, Hessler CS, Dhaliwal G, Betjemann JP, Fukuda KA, Alameddine LR et al. The AWOL tool: derivation and validation of a delirium prediction rule. J Hosp Med. 2013;8(9):493-9.

60. Kobayashi D, Takahashi O, Arioka H, Koga S, Fukui T. A prediction rule for the development of delirium among patients in medical wards: Chi-Square Automatic Interaction Detector (CHAID) decision tree analysis model. Am J Geriatr Psychatry. 2013;21(10):957-62.

61. Inouye SK. Predisposing and precipitating factors for delirium in hospitalized older patients. Dement Geriatr Cogn Disord. 1999;10(5):393-400.

62. Maviglia SM, Zielstorff RD, Paterno M, Teich JM, Bates DW, Kuperman GJ. Automating Complex Guidelines for Chronic Disease: Lessons Learned. J Am Med Inform Assoc. 2003;10(2):154-65.

63. Soman S, Zasuwa G, Yee J. Automation, Decision Support, and Expert Systems in Nephrology. Adv Chron Kidney Dis. 2008;15(1):42-55.

64. Khoury MJ, Ioannidis JP. Medicine. Big data meets public health. Science. 2014;346(6213):1054-5. 


\section{Chapter 7}

65. Croonen H. Big data in de zorg: niet alleen verzamelen. Medisch Contact. 2015;70(1).

66. Clynch N, Kellett J. Medical documentation: part of the solution, or part of the problem? A narrative review of the literature on the time spent on and value of medical documentation. Int J Med Inform. 2015;84(4):221-8.

67. van den Bemt BJF, Huiskes VJH. The medication therapy management pyramid shifting medication review to an integrated medication therapy management process. Eur J Hosp Pharm. 2015;22(4):219-221.

68. Wright A, Sittig DF. A four-phase model of the evolution of clinical decision support architectures. Int J Med Inform. 2008;77(10):641-9. 
Summary 

This thesis aims to improve the process of medication optimisation by exploring the use of a clinical decision support system (CDSS) regarding evidence-based pharmacotherapy. The process of medication optimisation consists of carrying out medication surveillance and medication reviews. Medication surveillance is defined as routinely giving specific advice to the patient and the physician by a pharmacist. Medication review is defined as a structured evaluation of a patient's medication by a physician and a pharmacist taking into account both the patient's medical history and laboratory values. The Dutch Health Care Inspectorate (IGZ) expects all residents of nursing homes and homes for the elderly to receive a medication review by a physician and a pharmacist twice a year and yearly, respectively. A CDSS is a computer program designed to support healthcare professionals in clinical decision-making such as medication surveillance. The first generation CDSSs generated many false positive alerts, which limits their value in the daily performance of medication surveillance, and therefore to date a lack of optimally automated support for medication reviews remains.

In chapter 1 , the introduction of the thesis, the study objectives of this thesis are introduced. The first objective, as described in chapter 2, was to identify the current execution as well as the extra efforts required to perform the above mentioned compulsory medication reviews, so that the need for automated support of the complex process of medication reviews could be properly identified.

The second study objective, as described in detail in chapter 3, was to determine which aspects are relevant for the improvement of the efficiency of an advanced CDSS used in medication surveillance.

The third objective of this thesis, as further described in chapter 5, was to explore the possibilities of using an advanced CDSS to support medication reviews. Finally, the fourth objective, as described in chapter 6 , was to explore the possibilities of using a CDSS for the prediction of an important subacute illness in hospitalised patients, i.e. the development of a predictive algorithm for delirium in hospitalised older adults.

Chapter 2 provides an overview of the clinical practice of medication reviews for older people in Dutch residential and nursing homes which are performed by pharmacists. A web-based survey was sent to 87 hospital pharmacists and 270 community pharmacists which was used to answer the following research questions:

1) To what extent are medication reviews performed as required by the IGZ?

2) How much time is needed to perform the required medication reviews?

3) In the opinion of the pharmacist, is the current way of medication reviewing economically efficient and medically relevant?

On the basis of the reported number of annually reviewed patients from each pharmacy we calculated that in a best-case scenario $76 \%$ of the IGZ-required medication reviews are performed, while in a worst-case scenario $42 \%$ of the IGZ-required medication reviews are performed. The pharmacies that participated in the survey would need to 
carry out a mean of 731 medication reviews per year and reported a mean of 29 minutes spent on one medication review. In each average pharmacy one pharmacist would therefore need to spend 2.5 months a year on medication reviews in order to comply with the IGZ requirements. Nevertheless, almost every pharmacist who filled out the survey, reported to regard the medication reviews as medically relevant, but only $47 \%$ considered them to be economically efficient at this moment. Due to the explorative nature of the survey and its limitation to pharmacists, the results do not provide a complete overview of the situation in the Netherlands and should be interpreted with caution. However, the results of the survey did show that the required medication reviews demand large amounts of time which means that more efficient methods are needed to be able to perform all the IGZ-required medication reviews adequately and feasibly. Automation of the medication review process might improve this efficiency.

Chapter 3 describes the first step in the automation of medication reviews, which consists of the identification and quantification of both the positive and the negative properties of a home grown advanced CDSS used in medication surveillance. The alerts generated by the home grown CDSS from the Zuyderland Medical Centre were extracted for the period September 2011 to December 2011. The number of generated alerts, the number of actions by pharmacists on the generated alerts, and the reasons why alerts had been classified as not relevant were analysed. The alerts that had been considered clinically relevant, were alerts which had prompted the pharmacist to contact the physician. The 4,065 alerts analysed from the chosen period were divided into three categories; 1,137 (28.0\%) newly generated alerts, 2,797 (68.8\%) repeat alerts and 131 (3.2\%) double alerts. Only 3.6\% of the total number of alerts had been considered to be clinically relevant. The reasons why $96.4 \%$ of the alerts had been classified as not clinically relevant were analysed, and these were grouped into: a) the dosage was correct or had already been adjusted, b) the drug had been (temporarily) stopped, c) the monitored laboratory value or drug dosage had already been reverted to a laboratory value within the reference limits or to a correct dosage. These reasons were linked to three categorical limitations of the home grown CDSS: 1) lack of criteria included in the algorithms leading to high amount of false positive alerts, 2) insufficient CDSS optimisation causing an unability to suppress alerts, and 3) manual data delivery to CDSS instead of continuous data delivery. These three categories have been identified as important for the development of an efficient advanced CDSS. Other requirements that we defined for the development of such a CDSS are: simple adjustment or implementation of criteria that would lead to the alerting of an algorithm, validation of the CDSS, easy implementation of the CDSS, use of recognised technical standards for sharing decision support content, easy use of the CDSS in daily practice, and supply of information by the CDSS on previous alerts and actions. The development and use of advanced CDSSs is a relatively new concept in hospitals and nursing homes, and therefore these CDSS requirements are not yet always integrated into the currently used CDSSs. Our study has 
undoubtedly shown which categories are important for the increase of the efficiency of a CDSS.

Chapter 4 describes the development of an advanced CDSS by using the criteria identified in chapter 3. The newly developed CDSS aims to function independently of prescribing software, and to monitor all prescribed drugs while at the same time taking into account co-medication, laboratory-data and co-morbidities.

It seems reasonable to assume that a manual reviewing tool is only as good as the health care professional using it. Outcomes will be influenced by variables such as knowledge of evidence-based guidelines or perhaps the focus of the healthcare professional. A CDSS seems to be able to support medication reviews in a reproducible way, and thus also able to reduce the time needed to perform medication reviews. Therefore we aimed to develop a CDSS that would increase the efficiency of medication surveillance, thereby reducing the false positive alerts and also providing alerts that could be used to support medication reviews.

The CDSS development phases were part of a larger study and involved: 1) development of the computerised system, 2) development of the clinical rules, 3) validation of the CDSS, 4) randomised controlled trial, and 5) feasibility study for implementation in different nursing homes.

The steps 1 to 3 were part of this thesis. The idea to automate medication reviews was not new, but our approach which does not require the manual input of patients' characteristics to perform medication reviews, was to the best of our knowledge described for the first time. We have developed a knowledge-based CDSS, named the 'Clinical Rule Reporter' which contains algorithms that alert to pre-defined problems by using decision tree rules. The CDSS has been developed to support both advanced medication surveillance and medication reviews.

Chapter 5 describes the potential of the newly developed CDSS to support the manually performed medication reviews. The first aim was to estimate the added value of a CDSS in the performance of medication reviews in hospitalised elderly people. The second aim was to identify the limitations of the newly developed CDSS by an analysis of the identified drug related problems (DRPs) in both the manual medication reviews and in the CDSS. The DRP notifications generated by the CDSS were compared with those identified by the manual medication reviews, thus allowing further improvement of the CDSS. The medication reviews were performed for 33 patients who had been admitted to the geriatric ward of the Zuyderland Medical Centre. Parallel to these medication reviews electronically available patient information was introduced into the CDSS. A total of 223 DRP strategies were identified during the manual medication reviews while the CDSS generated a total of 70 DRP clinically relevant notifications. Of the DRP notifications generated by the CDSS, $63 \%$ (44) were also found during the manual medication reviews and $37 \%$ (26) were newly identified DRPs.

The CDSS generated DRPs conveyed 28\% (70) of all 249 clinically relevant DRPs that were identified in the CDSS supported medication review. Classification according to the 
type of medication error reported by the DRP notifications revealed that mainly the medication errors 'contraindications/interactions/side effects' and 'indication without medication' were identified by the CDSS while these had not been identified during the manual medication reviews. In contrast to these results, the error types 'medication without indication', 'double medication', and 'wrong medication' were found by the manual medication review while most of these were not identified by the CDSS. The fact that these medication error types were not found by the CDSS, seems to be the result of the lack of input of documented multi-morbidity information from electronic health records, the under-development of deprescribing algorithms, and the underdevelopment of indicators that would enable a CDSS to interpret the progression or status of indications and adverse drug events. The newly developed CDSS used in this study is currently not able to replace the manual medication reviews, but it does offer added value for the manual medication reviews because certain DRPs that were not identified through the manual medication reviews, were indeed identified by the CDSS. It has become clear that the strengths and weaknesses of the current CDSS can be determined according to the medication error types, and these weaknesses (i.e. error types of unidentified DRPs) should be used to improve the CDSS.

Chapter 6 reports on the exploration of the use of a CDSS for other purposes than medication surveillance and medication reviews. A CDSS can also be used on an individual level for sub-acute disease risk prediction. If an individual's risk for a specific disease can be predicted and automatically presented, then personalised treatment strategies can be initiated on time. Delirium is a disorder of cognition and attention which may develop in older adults after hospitalisation, and an automated prediction model for delirium has clear relevancy for clinical practice. Delirium occurs frequently in hospitalised older adults and is associated with high costs and increased morbidity and mortality. Some important risk factors (e.g. cognitive disorders, vision impairment, lower functional status and illness severity) used in manual delirium prediction models, are currently not documented electronically for automation purposes. Nevertheless, it seems useful to develop an automated delirium risk prediction model which applies only the currently electronically available risk factors. In a retrospective cohort study we applied logistic regression to a database containing electronically available clinically relevant information on patients of 60 years or older who had developed a delirium during hospital admission. This study resulted in a "medication model" with an AUROC (area under the receiver operating characteristics curve) of 0.76 . Since an AUROC higher than 0.75 is regarded as clinically relevant, the developed model can be regarded as clinically relevant. This study showed that a CDSS can be used to predict the risk of a delirium in individual hospitalised patients by using electronically available predictive variables. To improve the quality of these predictive models, clinical risk factors such as patient reported outcomes and patients' characteristics should be documented, ready and complete, to be used for automation. When integrated in the electronic patient records, 
automated predictive medicine, such as this delirium prediction model, could in the future serve as a compass for the physician.

Chapter 7 provides a general discussion of the implications of the results presented in this thesis in relation to the current literature and with a view to future research and developments. 

Samenvatting 

In dit proefschrift wordt het gebruik van een digitaal systeem ter ondersteuning van klinische beslissingen (CDSS: Clinical Decision Support System) in het kader van rationele farmacotherapie onderzocht met als doel verbetering van het proces van optimalisatie van de medicatie. Het proces van medicatie-optimalisatie bestaat uit medicatiebewaking en medicatiebeoordelingen. Medicatiebewaking wordt gedefinieerd als het door een apotheker routinematig gepersonaliseerd advies geven aan de patiënt en de arts. Een medicatiebeoordeling wordt gedefinieerd als een gestructureerde evaluatie van de medicatie van een patiënt door arts en apotheker waarbij rekening wordt gehouden met de medische historie en laboratoriumgegevens van de patiënt. De Inspectie voor de Gezondheidszorg (IGZ) stelt verplicht dat alle bewoners van verpleeghuizen tweejaarlijks en alle bewoners van verzorgingshuizen jaarlijks een medicatiebeoordeling krijgen. Een CDSS is een computerprogramma dat ontworpen is om zorgverleners te ondersteunen bij beslissingen in de klinische praktijk zoals bij medicatiebewaking. De eerste generatie CDSS-en geven echter veel foutieve positieve meldingen bij de toepassing van medicatiebewaking waardoor de waarde van dit computerprogramma in de dagelijkse praktijk beperkt is. Er bestaat tot op heden dan ook nog geen optimaal werkend CDSS om medicatiebeoordelingen te ondersteunen.

In hoofdstuk 1, de introductie van dit proefschrift, worden de onderzoeksdoelstellingen uiteengezet. Het eerste doel, zoals beschreven in hoofdstuk 2, was het onderzoeken van het benodigde tijdsbeslag dat gemoeid is met het aantal hedendaags uitgevoerde medicatiebeoordelingen. De hier gevonden resultaten zullen worden gebruikt om de mate van inspanning die nodig is voor het uitvoeren van alle door de IGZ verplichte medicatiebeoordelingen te bepalen, om zo de noodzaak voor automatisering van medicatiebeoordelingen vast te stellen.

Het tweede doel, zoals beschreven in hoofdstuk 3, was het onderzoeken van de relevante aspecten voor de verbetering van een efficiënt tweede generatie (geavanceerd) CDSS toegepast voor medicatiebewaking.

Het derde doel van deze thesis, zoals beschreven in hoofdstuk 5, was het onderzoeken van de mogelijkheid voor het toepassen bij medicatiebeoordelingen van een efficiënt geavanceerd CDSS .

Het laatste, vierde doel, zoals beschreven in hoofdstuk 6, was het onderzoeken van de mogelijkheden om een efficiënt geavanceerd CDSS te gebruiken ter voorspelling van een ernstige subacute aandoening bij patiënten opgenomen in het ziekenhuis, waarbij specifiek de ontwikkeling van een algoritme is onderzocht dat een delirium kan voorspellen bij een populatie van patiënten met een hoge leeftijd.

Hoofdstuk 2 geeft een overzicht van de klinische praktijk van apothekers die medicatiebeoordelingen uitvoeren bij ouderen die zijn opgenomen in Nederlandse verpleeghuizen en verzorgingshuizen. Een web-based vragenlijst is verstuurd naar 87 ziekenhuisapothekers en 270 openbare apothekers om de volgende onderzoeksvragen te beantwoorden: 
1) In welke mate worden medicatiebeoordelingen uitgevoerd zoals verplicht door de IGZ?

2) Hoeveel tijd er nodig is om de IGZ verplichte medicatiebeoordelingen uit te voeren?

3) Zijn de huidige methodes van medicatiebeoordelingen economisch efficiënt en medisch relevant volgens de apotheker?

Op basis van de gerapporteerde jaarlijks beoordeelde patiënten is berekend dat door elke apotheek er maximaal $76 \%$ en minimaal $42 \%$ van de door de IGZ verplichte medicatiebeoordelingen worden uitgevoerd. De aan de vragenlijst deelnemende apotheken zouden gemiddeld genomen 731 medicatiebeoordelingen per jaar moeten uitvoeren waarbij gemiddeld genomen 29 minuten nodig is voor het uitvoeren van één medicatiebeoordeling.

In elke gemiddelde apotheek zou één apotheker 2.5 maanden nodig hebben om de door de IGZ verplichte medicatiebeoordelingen uit te voeren. Echter, bijna elke deelnemende apotheker achtte de medicatiebeoordelingen medisch relevant; maar slechts $47 \%$ oordeelde de medicatiebeoordelingen als economisch efficiënt.

Door de verkennende opzet van deze vragenlijst, die uitsluitend is uitgevoerd onder apothekers, geven de resultaten van dit onderzoek geen sluitend overzicht van de situatie van medicatiebeoordeling bij verpleeg- en verzorgingshuizen in Nederland. De resultaten moeten daarom met enige terughoudendheid worden geïnterpreteerd. Daarentegen laat deze vragenlijst wel zien dat de medicatiebeoordelingen een groot tijdsbeslag vergen. Om alle IGZ verplichte medicatiebeoordelingen adequat en volledig uit te voeren is er een meer efficiënte methode nodig. Automatiseren van het proces van medicatiebeoordelingen kan mogelijk bijdragen aan die efficiëntie.

Hoofdstuk 3 beschrijft de eerste stap in de automatisering van medicatiebeoordelingen: de identificatie en kwantificering van de positieve en negatieve eigenschappen van een lokaal ontwikkelt geavanceerd CDSS ten behoeve van medicatiebewaking. De onderzochte signalen van het CDSS in het Zuyderland Medisch Centrum zijn afkomstig uit de periode september 2011 tot december 2011. Het aantal gegenereerde signalen, het aantal ondernomen acties van apothekers op deze signalen en de redenen waarom de signalen als niet klinisch relevant werden beschouwd, zijn geanalyseerd.

De signalen werden als relevant beschouwd zodra de apotheker de arts contacteerde. De 4065 geanalyseerde signalen zijn onderverdeeld in: 1137 (28.0\%) nieuwe, gegenereerde signalen, 2797 (68.8\%) herhaalde signalen en 131 (3.2\%) dubbele signalen. Slechts $3.6 \%$ van het totaal aantal signalen werd als klinisch relevant beoordeeld. De redenen waarom $96.4 \%$ van de signalen werd geclassificeerd als niet klinisch relevant, zijn ook geanalyseerd en als volgt gegroepeerd: a) de dosering was correct of reeds aangepast, b) het geneesmiddel was (tijdelijk) gestopt, c) de van toepassing zijnde laboratoriumwaarde of de dosering was reeds teruggegaan naar de normale waarde. Deze 
redenen zijn gekoppeld aan drie categorische beperkingen van het lokaal ontwikkeld geavanceerd CDSS: 1) tekortkoming van criteria in de algoritmen leidend tot een hoog aantal van foutieve positieve signalen. 2) onvoldoende mogelijkheden tot systeemoptimalisatie met als gevolg dat onnodige signalen niet kunnen worden onderdrukt, en 3) het handmatig inlezen van bulkdata in het systeem welke continue aangeleverd zouden moeten worden. Deze drie categorieën zijn geïdentificeerd als belangrijk voor de ontwikkeling van een efficiënt geavanceerd CDSS. Andere gedefinieerde voorwaarden voor de ontwikkeling van een efficiënt geavanceerd CDSS zijn: het eenvoudig kunnen aanpassen of implementeren van criteria die een signaal initiëren; validatie van een CDSS; de eenvoudige invoering van een CDSS; het gebruiken van erkende technische standaarden voor het delen van de inhoud die gebruikt is in de beslissingsondersteuning; het eenvoudig kunnen gebruiken van het CDSS in de dagelijkse praktijk en het informeren door het CDSS van eerder afgehandelde signalen.

De ontwikkeling en het gebruik van een geavanceerd CDSS is een relatief nieuw concept in ziekenhuizen en verpleeghuizen met als gevolg dat de bovenstaande voorwaarden nog niet (altijd) worden toegepast in het gebruikte CDSS. Dit onderzoek toont duidelijk aan welke categorieën van belang zijn om de efficiëntie van een CDSS te laten toenemen.

Hoofdstuk 4 beschrijft de ontwikkeling van een geavanceerd CDSS waarbij de criteria benoemd in hoofdstuk 3, zijn toegepast. Het nieuw te ontwikkelen CDSS beoogt onafhankelijk van verschillende voorschrijfsystemen alle voorgeschreven geneesmiddelen te monitoren, waarbij rekening wordt gehouden met co-medicatie, laboratoriumwaarden en comorbiditeiten.

Het lijkt redelijk om aan te nemen dat bij het uitvoeren van een handmatige medicatiebeoordeling, de kwaliteit wordt bepaald door de zorgverlener die de beoordeling uitvoert, al dan niet met een ondersteunend hulpmiddel. Resultaten van de medicatiebeoordeling zijn afhankelijk van variabelen zoals kennis van richtlijnen of zelfs de focus van de zorgverlener. Het lijkt mogelijk dat een geavanceerd CDSS de medicatiebeoordelingen op een reproduceerbare wijze kan ondersteunen, waarbij de benodigde tijd voor een dergelijke beoordeling ook wordt verminderd. Op basis hiervan hebben wij beoogd een CDSS te ontwikkelen dat het aantal fout-positieve en fout-negatieve signalen minimaliseert en dus de efficiëntie van de medicatiebewaking zal verhogen, welke tegelijkertijd een basis vormt voor het ondersteunen van medicatiebeoordelingen.

De ontwikkelingsfases van het CDSS zijn onderdeel van een onderzoeksproject en omvatten: 1) de ontwikkeling van het computersysteem, 2) de ontwikkeling van de beslissingsondersteunende algoritmen (clinical rules), 3) validatie van het CDSS, 4) een gerandomiseerde studie, 5) een haalbaarheidsonderzoek voor implementatie in diverse verpleeghuizen. Stap 1 tot en met 3 zijn onderdeel van dit proefschrift.

Het concept om medicatiebeoordelingen te automatiseren is geen nieuw concept, echter onze aanpak, waarbij de handmatige invoer van patiënten gegevens niet nodig is, is door ons voor het eerst beschreven. We hebben een 'knowledge-based' CDSS 
ontwikkeld, genaamd de 'Clinical Rule Reporter', dat algoritmen volgens het principe van een beslisboom bevat om daarmee vooraf gedefinieerde geneesmiddelgerelateerde problemen te signaleren. Het CDSS is ontwikkeld om geavanceerde medicatiebewaking en medicatiebeoordelingen te ondersteunen.

Hoofdstuk 5 beschrijft het onderzoek naar de mogelijkheid van het nieuw ontwikkelde CDSS om de handmatig uitgevoerde medicatiebeoordelingen te ondersteunen. Het eerste doel was het bepalen van de toegevoegde waarde van het CDSS in het ondersteunen van medicatiebeoordelingen bij ouderen opgenomen in het ziekenhuis. Het tweede doel was het identificeren van de beperkingen van het nieuw ontwikkelde CDSS met behulp van een analyse van de geïdentificeerde geneesmiddel-gerelateerde problemen (DRPs) die zowel met de handmatige medicatiebeoordeling als met het CDSS zijn gevonden. De door het CDSS geïdentificeerde DRPs zijn vergeleken met de in de handmatige medicatiebeoordelingen vastgestelde DRPs, om een verdere verbetering van het CDSS mogelijk te maken.

De medicatiebeoordelingen zijn uitgevoerd bij 33 patiënten die waren opgenomen op de geriatrische afdeling van het Zuyderland Medisch Centrum. Gelijktijdig aan deze medicatiebeoordelingen is de aanwezige elektronische patiëntinformatie in het CDSS geladen. Een totaal van 223 DRP strategieën zijn vastgesteld tijdens de handmatige medicatiebeoordeling, terwijl het CDSS 70 klinisch relevante signalen heeft gegenereerd.

Van de door het CDSS gegenereerde signalen was 63\% (44) ook vastgesteld tijdens de handmatige medicatiebeoordelingen, terwijl 37\% (26) van de signalen, nieuwe, niet eerder vastgestelde geneesmiddel-gerelateerde problemen waren. De door het CDSS gegenereerde signalen vormen $28 \%$ (70) van alle 249 klinische relevante DRPs die zijn geïdentificeerd en vastgesteld door de combinatie van het CDSS en de handmatige medicatiebeoordeling.

Classificatie van de gevonden geneesmiddel-gerelateerde problemen aan de hand van het type medicatiefout onthulde dat met name 'contra-indicaties/interacties/bijwerkingen' en 'indicaties zonder voorgeschreven medicatie' werden geïdentificeerd door het CDSS, terwijl deze niet werden vastgesteld tijdens de handmatige medicatiebeoordeling.

De medicatiefouten 'medicatie voorgeschreven zonder aanwezige indicatie', 'voorgeschreven dubbele medicatie', 'voorgeschreven verkeerde medicatie' werden met name vastgesteld in de handmatige medicatiebeoordeling, terwijl deze meestal niet werd geïdentificeerd door het CDSS.

De tekortkomingen van het CDSS in het identificeren van medicatiefouten lijkt een gevolg van: te weinig inbreng van multimorbiditeiten uit het elektronische patiëntdossier (EPD) de afwezigheid van algoritmen die het stoppen van geneesmiddelen adviseren en de afwezigheid van indicatoren die de ontwikkeling van een morbiditeit of bijwerking meewegen. Het nieuw ontwikkelde CDSS dat is onderzocht in deze studie, kan momenteel nog niet de handmatige medicatiebeoordeling vervangen, echter het heeft wel toegevoegde waarde, omdat het CDSS wel DRPs heeft geïdentificeerd, die niet zijn vastgesteld tijdens de handmatige medicatiebeoordeling. Aangetoond is dat zowel de 
sterke als de zwakke punten van het huidige CDSS kunnen worden bepaald met behulp van het type geïdentificeerde medicatiefouten. De zwakke punten van het CDSS, d.w.z. de medicatiefouten die niet zijn geïdentificeerd door het CDSS, kunnen worden gebruikt om het CDSS verder te verbeteren.

Hoofdstuk 6 beschrijft de verkenning van het gebruik van een CDSS voor andere doeleinden dan medicatiebewaking en medicatiebeoordelingen. Een CDSS kan ook worden gebruikt op een individueel niveau ten behoeve van de risicovoorspelling van subacute ziekten. Als het risico van een individu voor specifieke ziekten automatisch gesignaleerd kan worden, dan kunnen gepersonaliseerde behandelingsstrategieën tijdig geïnitieerd worden. Delier is een aandoening van de cognitie en aandacht. De geautomatiseerde voorspelling van een delier heeft overduidelijke voordelen in de klinische praktijk. Een delirium komt vaak voor onder ouderen opgenomen in het ziekenhuis en is geassocieerd met hoge kosten, toegenomen morbiditeit en mortaliteit. Hoewel sommige belangrijke risicofactoren voor het ontwikkelen van een delier, zoals cognitieve aandoeningen, visie beperkingen, verminderd functioneren en de mate van ziekte, gebruikt worden bij handmatige voorspellingsalgoritmen, worden deze tot op heden niet elektronisch vastgelegd ten behoeve van automatisering. Desondanks lijkt het zinvol om een geautomatiseerd deliriumrisico voorspellingsmodel te ontwikkelen dat exclusief gebruik maakt van de op dit moment voor automatiseringsdoeleinden aanwezige risicofactoren.

In een retrospectieve cohortstudie, hebben we logistische regressie analyse toegepast op een dataset met daarin patiënten van 60 jaar of ouder, die een delirium ontwikkelden tijdens de ziekenhuisopname. Dit heeft geresulteerd in een model met een AUROC (area under the receiver operating characteristics curve) van 0.76 . Het ontwikkelde model kan worden beschouwd als klinisch relevant, omdat de AUROC hoger is dan 0.75.

Deze studie laat zien dat een CDSS kan worden gebruikt om het risico van een delier geautomatiseerd te voorspellen voor elke in een ziekenhuis opgenomen patiënt door uitsluitend de aanwezige elektronische beschikbare risicofactoren te gebruiken. Om de kwaliteit van voorspellingsmodellen te verbeteren zouden klinische risicofactoren zoals patiënt-gerapporteerde uitkomsten en relevante patiënteninformatie volledig moeten worden vastgelegd teneinde automatisering beter te ondersteunen. Het integreren van geautomatiseerde voorspellingen in een EPD, zoals een delirium voorspellingsmodel, kan in de toekomst als een kompas voor de zorgverlener dienen.

Hoofdstuk 7 beschrijft een algemene discussie waarbij de gevonden resultaten uit dit proefschrift in perspectief tot de huidige literatuur worden geplaatst, waarbij tevens mogelijkheden voor toekomstige ontwikkelingen en onderzoek worden besproken. 

Valorisation 

The simultaneous use of many drugs is called polypharmacy, which is known to increase as people get older. Polypharmacy is usually defined as the concomitant use of five or more drugs. Previous research has shown that polypharmacy increases the risk of drug interactions, adverse effects, and often causes problems with patient compliance. In addition, it can lead to unnecessary hospitalisation (1-5).

A clinical decision support system (CDSS), which is a computer program designed to support healthcare professionals in clinical decision-making, has already provided a feasible solution for a basic form of medication surveillance.

Medication surveillance, one of the activities carried out by physicians and pharmacists to assure safe and beneficial use of drugs, has already been performed since several decades by using a first generation CDSS. This first generation CDSS alerts against incorrect dosages, drug-drug interactions, and contraindications (drug-disease interactions). The first generation CDSS can nowadays be combined with one of the more advanced second generation CDSSs that are able to combine information on individual patient characteristics such as diagnoses and laboratory values.

Currently, in addition to the daily (digital) medication surveillance intended to prevent incorrect dosages, drug-drug interactions, and contraindications, (manual) medication reviews of older adults are performed periodically to ensure optimal pharmacotherapy. A 'medication review' is defined as a structured evaluation of a patient's medication by a physician and a pharmacist, taking into account both the patient's medical history and laboratory values (6). In the Netherlands, the Health Care Inspectorate (IGZ) expects all residents of nursing homes and homes for the elderly to receive a medication review by a physician and a pharmacist twice a year and yearly, respectively.

Both processes, medication surveillance and medication reviews, aim for medication optimisation, which is the optimal use of medication by taking into account the benefits and safety aspects of the drugs used. This thesis has explored the possibilities of using an advanced CDSS to automate medication optimisation.

In the following paragraphs the societal relevance of the thesis will be shown in a number of highlighted messages for daily practice, each of which is subsequently explained in more detail.

The results of this thesis confirm that a more feasible and efficient method is essential to fulfil the current IGZ-required medication reviews.

In a survey conducted among pharmacists who provide care for nursing homes and residential homes, we have demonstrated that only a part of the medication reviews required by the IGZ is actually performed with a minimum performance of $42 \%$ and a maximum of $76 \%$ with respect to the IGZ-requirements. The enormous effort needed to comply with the required medication reviews is most probably the cause of this underperformance. 
We have shown that in an average pharmacy one pharmacist annually has to work full-time for 2.5 months to perform the IGZ required medication reviews. This suggests that performing all the medication reviews required by the IGZ is very time consuming and costly when performed with the currently available manual methods.

This thesis shows that automation of medication reviews may be useful. The study provides practical insight into the aspects that have to be taken into consideration and need to be optimised in order to further improve the efficiency of these reviews.

Our study indicated that automation of medication reviews can provide efficiency benefits. The idea to automate medication reviews is not new. However, our proposed approach, which does not require the manual input of individual patient data, e.g. medication and patients' characteristics, into the CDSS, followed by automated generation of drug related alerts, will most probably lead to a considerable reduction of the review time.

The first step towards the automation of medication reviews by a CDSS was to increase the efficiency of the medication surveillance of an advanced CDSS, which is pivotal in reducing alert fatigue and in being able to perform cost-efficient medication surveillance. Performing automated medication surveillance with an efficient advanced CDSS may already contribute to the optimisation of drug use, and should actually prevent suboptimal drug use that would normally only be identified through manually performed medication reviews.

The ideal advanced CDSS should optimally alert for all clinical relevant drug related problems without any false positive alerts, thereby already preventing alert fatigue. However, it might be more realistic to aim for a CDSS that alerts for all clinical relevant drug related problems of which $80 \%$ are clinically relevant.

The second step in the automation of medication reviews by a CDSS was the development of a CDSS with content that actually supports medication reviews, i.e. algorithms to identify drug related problems (DRPs). In the thesis we have described the necessary steps for the development of such a CDSS.

This thesis shows that automation of medication reviews by a comprehensive CDSS should be further improved by increasing the number of relevant algorithms that identify drug related problem. This increase in relevant algorithms is necessary to widely implement the CDSS in patient care.

The third step was to explore the value of the newly developed CDSS by comparing the performance of manual medication reviews in hospitalized geriatric patients with the DRP notifications generated by the CDSS. This comparison showed that the new CDSS 
was able to support medication reviews by alerting for $10 \%$ of newly identified DRPs in relation to the DRPs previously identified in the manual medication reviews. However, in our study the overall support of the manual medication review remained limited to $28 \%$ of the total of identified DRPs, i.e. DRPs identified by the manual medication review and CDSS, thereby revealing that our new CDSS needs to be enhanced. On the other hand, the CDSS algorithms that had identified the DRPs, had a sensitivity of $73 \%$ and specificity of $99 \%$, which is good for an advanced CDSS. Therefore, the CDSS studied is in itself already a very efficient tool to augment the first generation CDSS which was primarily used for medication surveillance. Furthermore, our study also confirmed that the categories identified are relevant for an efficient CDSS that claims to be both feasible and effective for performing automated medication reviews. Although the new CDSS should be further enhanced in order to provide optimal support for medication reviews, the DRPs that have already been identified by the CDSS, can even now be easily applied on a daily or weekly basis instead of the manual medication reviews performed once or twice per year. When the CDSS will be applied more frequently, this will allow early prevention and will increase the efficiency of medication reviews. Moreover, the already automated parts of the medication review in the CDSS have now been supported by the addition of literature references, thus allowing the healthcare provider to estimate the value of the alerts.

Nevertheless, the limitations of the newly developed CDSS, i.e. undetected DRPs and irrelevant alerts, should be solved so that the number of relevant DRPs identified by the CDSS can be further increased. In addition, in our study we have characterized DRPS that are not easily automated and implemented into a CDSS, i.e. algorithms that initiate deprescribing. These DRPs, and the other described limitations, offer a roadmap of important issues that should be investigated further to improve the CDSS even more. When eventually it will be possible to automate medication reviews to a maximum, this will create the possibility to spend more time on the non-automated parts of the medication review process, such as the consultations between physician, pharmacist, and patient.

This thesis has revealed that a CDSS may also be used in the detection of patients prone to develop a delirium.

Finally, the use of a CDSS in the prediction of a sub-acute disease was also explored in this thesis. In 18 European countries 182 billion US dollars is spent yearly on care for delirium patients alone, while $30-40 \%$ of the delirium cases might have been prevented and delirium diagnoses are often missed (7). Delirium occurs frequently in hospitalised older adults, and is associated with increased morbidity and mortality.

Our study revealed that a CDSS can indeed be used to automatically predict a delirium. Despite the absence of important electronically available risk factors, the devel- 
oped prediction model showed to be already worthwhile with only the electronically available delirium risk factors. The absence of important risk factors uncovered the limitations of the currently available information in electronic health records. In general there is a lack of recorded documentation of relevant patient information in electronic health records (8). If this patient information, including relevant risk factors, would be available for automation, then the predictive quality of the developed delirium model developed during our study would increase even more.

Furthermore, automated presentation of patients at risk for delirium would be very beneficial since it does not seem realistic to assume that every physician has similar expertise on high impact diseases such as delirium. Automated prediction models do not have to be limited to delirium, but can also be used more widely for other prediction algorithms such as the risk of falling or the risk of neonatal sepsis. In the case of an increased risk of falling, additional preventive measures could be initiated in time or medication causing the fall risk could be minimised. In the case of the early alerting for neonatal sepsis by automated prediction, this has already been shown to decrease mortality $(9,10)$.

This type of applicability of a CDSS may be very useful in the future to improve patient care and patient safety, although more research is needed to fully appreciate advanced CDSSs for the use in the automated prediction of delirium and other relevant diseases or care problems. 


\section{REFERENCES}

1. Maher RL, Hanlon J, Hajjar ER. Clinical consequences of polypharmacy in elderly. Expert Opin Drug Saf. 2014;13(1):57-65.

2. Gandhi TK, Weingart SN, Borus J, Seger AC, Peterson J, Burdick E et al. Adverse drug events in ambulatory care. N Engl J Med. 2003;348(16):1556-64.

3. Tsai KT, Chen JH, Wen CJ, Kuo HK, Lu IS, Chiu LS et al. Medication adherence among geriatric outpatients prescribed multiple medications. Am J Geriatr Pharmacother. 2012;10(1):61-8.

4. Field TS, Gurwitz JH, Avorn J, McCormick D, Jain S, Eckler M et al. Risk factors for adverse drug events among nursing home residents. Arch Intern Med. 2001;161(13):1629-34.

5. Nobili A, Pasina L, Tettamanti M, Lucca U, Riva E, Marzona I et al. Potentially severe drug interactions in elderly outpatients: results of an observational study of an administrative prescription database. J Clin Pharm Ther. 2009;34(4):377-86.

6. Zermansky A, Petty D, Raynor T, Lowe C, Freemantle N. Clinical medication review by a pharmacist of patients on repeat prescriptions in general practice: a randomised controlled trial. Health Technol Assess. 2002;6(20):86.

7. Inouye SK, Westendorp RG, Saczynski JS. Delirium in elderly people. Lancet. 2014;383(9920):911-22.

8. Hope C, Estrada N, Weir C, Teng CC, Damal K, Sauer BC. Documentation of delirium in the VA electronic health record. BMC Res Notes. 2014;7:208.

9. Fairchild KD. Predictive monitoring for early detection of sepsis in neonatal ICU patients. Curr Opin Pediatr. 2013;25(2):172-9.

10. Deschamps T, Le Goff CG, Berrut G, Cornu C, Mignardot J-B. A decision model to predict the risk of the first fall onset. Exp Gerontol. 2016;81:51-5. 

Dankwoord 

Beste collega's, vrienden en familie, dit proefschrift is in de afgelopen jaren tot stand gekomen. Tijdens besprekingen, feestjes, verhuizingen, klussen en zelfs op vakanties aan de andere kant van de wereld is erover gesproken, nagedacht en aan gewerkt. ledereen heeft zich tijdens zo'n traject wel eens afgevraagd: 'is dat het allemaal wel waard?'. Jullie mogen dit zelf invullen met het boekje in je handen en ná 30 november. Echter zonder jullie steun, in elke vorm, was dit boekje er niet gekomen zoals het er nu ligt. ledereen die op een bepaalde manier: subtiel, onbewust of juist heel nadrukkelijk heeft bijgedragen, wil ik bedanken voor zijn of haar bijdrage.

Ik wil echter een aantal van jullie in het bijzonder bedanken. Beginnend bij de harde kern, het promotieteam:

Beste Jos (Schols), zonder jou was deze dag er niet geweest. Je hebt me geholpen door dik en dun, en onvermoeibaar. Ik had me geen betere promotor kunnen wensen. Ik heb veel van je geleerd over de wetenschap en het schrijven zelf. Heel erg bedankt!

Beste Rob (Janknegt), met jouw humor en creatieve ideeën zijn we de onderzoeksmeetings voortreffelijk doorgekomen. Bedankt voor je revisies en frisse ideeën. Daarnaast heb ik veel geleerd van je relativerende blik op ogenschijnlijke grote problemen.

Beste Paul-Hugo (van der Kuy), samen met Rob ben je de 'vader' van dit onderzoeksproject. Zonder jullie visie op medicatiebeoordeling en medicatiebewaking was dit proefschrift er niet. Ook wil ik je bedanken voor alle kansen die jij mij hebt gegeven. Allereerst door mij in opleiding te nemen als ziekenhuisapotheker, terwijl ik uitsluitend ervaring als openbare apotheker had. Vervolgens was er de mogelijkheid onderzoekservaring op te doen met als ultiem resultaat dit proefschrift. Ook ben je mijn opleider voor de aantekening tot klinisch farmacoloog, waar ik veel heb geleerd van jou visie hierover. Je staat altijd klaar om te helpen, je hebt altijd ideeën en een oplossing voor elk probleem. Bedankt!

Beste Bjorn (Winkens), elke promotiestudent wenst zich een goede statisticus zoals jij bent. In jou heb ik een blind vertrouwen. Je bent een co-promotor die echt meedenkt en zich heel goed kan verplaatsen in de situatie van de promovendus. Ik heb enorm veel van je geleerd en ik ervaar onze contacten niet alleen zakelijk maar ook vriendschappelijk.

Naast het promotieteam wil ik ook graag de leden van de beoordelingscommissie, prof. dr. J.F.M. Metsemakers, prof. dr. O. Bekers, prof. dr. A.C.G. Egberts, prof. dr. R.J. van Marum, prof. dr. C. Neef, hartelijk danken voor de inhoudelijke kritische beoordeling van mijn manuscript. Dit heeft een toegevoegde waarde voor mij gehad. 
Als onderdeel van het onderzoeksproject hebben we regelmatige gesprekken en brainstormsessies gehad waarvan de aanwezige leden, veelal co-auteurs, dit proefschrift mede vorm hebben gegeven.

Beste Frans (Verhey), ik heb jouw bijdrage in de brainstormsessies en revisies van de stukken als zeer weloverwogen en belangrijk ervaren. Dank voor je scherpe analyses.

Beste Wubbo (Mulders), ook jouw bijdrage in de brainstormsessies en vele revisies, van met name de eerste artikelen, zijn van belang geweest in de ontwikkeling van mij als onderzoeker en auteur. Dank hiervoor.

Beste Kim (Hurkens), jij bent mij al voorgegaan en hebt de aftrap gegeven door als eerste te promoveren op dit onderzoeksproject. Jouw inbreng als arts, is zeer waardevol geweest. Tijdens de afgelopen jaren hebben wij op een efficiënte manier samengewerkt als collega onderzoekers.

Beste Carlota (Mestres Gonzalvo), CM, ik denk dat wij beiden het gevoel hebben dat we in hetzelfde schuitje zitten. We helpen elkaar dan ook ongevraagd wanneer nodig. Ik ben nu bijna klaar en ik wens je al het beste bij jouw laatste loodjes. Naast collega apotheker en mede-onderzoeker, ben je vooral een goede vriendin. Dank voor je steun en leuke, grappige en soms bizarre gesprekken.

Beste alle andere collega apothekers, sommige van jullie zijn actief betrokken bij het onderzoek geweest of zijn onderdeel van het onderzoeksproject van dit proefschrift.

Beste Brigit (van Oijen), we go way back! Wij kennen elkaar al vanaf het begin van de studie farmacie in Utrecht. In hetzelfde vriendengroepje hebben wij vele leuke momenten meegemaakt. Dankzij jou heb ik besloten om in Sittard te solliciteren voor de opleiding tot ziekenhuisapotheker. En het heeft me veel goeds gebracht. Ook wij zitten dus al lange tijd in hetzelfde schuitje. Ik ben dankbaar dat ik een collega heb, die mij goed kent zoals ik ben.

Beste Wong (Dennis), als mijn informele opleider tijdens mijn opleiding tot ziekenhuisapotheker en nu als formele mede-opleider voor de aantekening tot klinisch farmacoloog sta je altijd klaar om mij te helpen, of om met mij te sparren. Daarnaast ben je als collega voor mij zeer waardevol en als vriend met dezelfde interesses (sporten!) een geweldige gesprekspartner.

$\mathrm{Na}$ het afronden van mijn opleiding tot ziekenhuisapotheker ben ik gestart in het voormalige Atrium Medisch Centrum te Heerlen. Zodra ik de opleiding had afgerond begon ik fris en enthousiast aan een nieuwe baan. In mijn geval was dat bij een organisatie die 
voornemens was om te fuseren met mijn 'oude' opleidingsplek, het voormalige Orbis Medisch Centrum te Sittard. In dit fusietraject heb ik samen met Marga (Schols) intensief opgetrokken om het nieuwe gezamenlijke EVS te implementeren. In deze periode was ik ook nog wekelijks aanwezig in Sittard voor de opleiding tot klinisch farmacoloog. Het was een uitdagende tijd.

Beste André (Krings), ik denk dat jij goed hebt ingezien dat het implementeren van het EVS in een fuserende organisatie voor mij een interessant, uitdagend en leerzaam traject was. Heel erg bedankt voor je steun en begrip in deze soms roerige tijden. Het implementeren van een nieuw EVS met een go-live op één dag was een mooie ervaring.

Ik spreek ook mijn dank uit naar alle andere collega ziekenhuisapothekers (i.o.): Mark Reinders, Christian Guillaume, Lyonne van Rossum, Marga Schols, Kobra Waizy, Niels Boone, Vanja Milosevic, Zohreh Safipour, Fieke van Moorsel, Hans Cremers, Claudia Heijens, Peter Dekkers, Kimberly Shudofsky, Kim Keijzers, Judith van Dalem, Margriet van Dijk, Eugene Hardy en Piet Hooymans, die mij in soms drukke tijden hebben gesteund, geholpen of gewoon interesse hebben getoond in de voortgang van het onderzoek. Ik wens degenen die bezig zijn of stiekem plannen hebben voor ook een boekje, heel veel succes toe.

Uiteraard dank ik ook de medewerkers van de twee locaties voor de prettige samenwerking in relatie tot het onderzoek: Walther Sipers, Machiel Smid, Chantal de Weert, ik heb veel van jullie geleerd tijdens de wekelijkse medicatiereviews.

Ook de andere collega's bij wie ik veelvuldig heb aangeklopt voor een zoveelste extractie van gigantische hoeveelheden aan data: Sander Mulders en Armand Meertens bedankt!

Ook alle apothekersassistentes, managers en medewerkers van de beide locaties van de ziekenhuisapotheek wil ik graag bedanken. Ik werk met jullie allemaal intensief samen en ik vind het erg belangrijk dat we ondanks drukke en hectische tijden altijd ruimte hebben voor goede grappen.

De collega's van Digitalis en ClinicalRules.nl, zonder jullie was de Clinical Rule Reporter er niet eens geweest! Het is een prachtig systeem geworden.

Beste Bob (v.d. Loo), ik heb altijd met veel plezier met je samengewerkt, waarbij ik altijd de indruk heb gehad dat de 'uitdagingen' als sneeuw voor de zon verdwenen. Bedankt voor je hulp en je blijvende strijdvaardigheid. 
De belangrijkste personen in mijn leven die echt voor mij hebben gezorgd.

Lieve Bart en Cees, beste broers, beste vrienden, als ik niet jullie beiden vraag om advies, dan is het wel één van jullie twee (afhankelijk van het onderwerp;). Jullie zijn mijn voorbeelden, bedankt voor de nuchterheid en steun. Als oudere broers hebben jullie mij altijd, voor zover dat mogelijk was, geholpen. Ik hoop dat jullie weten dat ik er ook voor jullie ben zodra en voor zolang dat nodig is.

Lieve Papa en Mama, de liefste ouders die ik me kan voorstellen; bedankt voor alles wat jullie geven en gegeven hebben. Het kan niet beter, ik zou niet weten hoe, en ik hoop dat ik het ooit ook zo zal doen.

Allerliefste, lieve Simone, ik ben ontzettend blij met de manier waarop je mij geholpen hebt: het doorlezen van de stukken, het accepteren dat soms het werk aan het proefschrift voorging. Ook jij bent bezig met je proefschrift, ik hoop dat ik jou net zo goed kan helpen als jij mij geholpen hebt. Op de momenten dat ik het even niet meer wist, dan wist jij het wel. Op de momenten dat ik even geen geduld meer had, dan had jij het wel. Je bent m'n maatje, bedankt lief. 
List of publications 

1. Mestres Gonzalvo C, de Wit HAJM, Hurkens KPGM, van Oijen BPC, Janknegt R, Schols JMGA, Mulder WJ, Verhey FR, Winkens B, van der Kuy PHM. SCREAM (Supporting Clinical Rules Engine in the Adjustment of Medication).Submitted for publication.

2. Hurkens K.P.G.M., de Wit HAJM, Mestres Gonzalvo C, Janknegt R, Verhey F, Schols JMGA Stehouwer CDA, Winkens B, Mulder WJ, Magdelijns F, Scheeper M, van der Kuy PHM. The additional value of computer assisted medication review in hospitalised patients. Submitted for publication.

3. de Wit HAJM, Hurkens KPGM, Mestres Gonzalvo C, Winkens B, Smid M, Janknegt R, van der Kuy PHM, Schols JMGA.The support of a medication review in hospitalized patients by a computerized decision support system. SpringerPlus.2016;5(1):871.

4. de Wit HAJM, Winkens B, Mestres Gonzalvo C, Hurkens KPGM, Mulder WJ, Janknegt R, Verhey FR, van der Kuy PHM, Schols JMGA. The development of an automated ward independent delirium risk prediction model. Int J Clin Pharm. 2016;38(4):915-23.

5. Vossen LM, van Dongen CMP, Schoutteten MK, de Wit HAJM, de Leeuw PW. Survival after intoxication with inhaled methanol. Neth J Med. 2015;73(6).

6. de Wit HAJM, Mestres Gonzalvo C, Cardenas J, Derijks HJ, Janknegt R, van der Kuy $\mathrm{PHM}$, et al. Evaluation of clinical rules in a standalone pharmacy based clinical decision support system for hospitalized and nursing home patients. Int J Med Inform. 2015;84(6):396-405.

7. Mestres Gonzalvo C, Hurkens KPGM, de Wit HAJM, van Oijen BPC, Janknegt R, Schols JMGA, Mulder WJ, Verhey FR, Winkens B, van der Kuy PHM.To what extent is clinical and laboratory information used to perform medication reviews in the nursing home setting? the CLEAR study.Ther Clin Risk Manag. 2015;11:767-777.

8. Hurkens KPGM, Mestres Gonzalvo C, de Wit HAJM, van der Kuy PHM, Janknegt R, Verhey FR, Schols JMGA, Stehouwer CDA, Winkens B, Mulder WJ. Usually available clinical and laboratory data are insufficient for a valid medication review: a crossover study. J Nutr Health Aging. 2016;20(1):71-76.

9. de Wit HAJM, Winkens B, Mestres Gonzalvo C, Hurkens KPGM, Janknegt R, Schols JMGA, et al. Clinical practice of medication reviews in institutional care settings for older people in the Netherlands: an explorative survey. Eur J Hosp Pharm. 2015;22(4):212-218.

10. de Wit HAJM, Mestres Gonzalvo C, Hurkens KPGM, Mulder WJ, Janknegt R, Verhey FR, Schols JMGA, van der Kuy PHM. Development of a computer system to support medication reviews in nursing homes. Int J Clin Pharm. 2013;35(5):668-72

11. de Wit HAJM, Mestres Gonzalvo C, Janknegt R, Schols JMGA, van der Kuy PHM. A fully automated medication review? Int J Clin Pharm. 2014;36(2):220-1. 
12. Hurkens KPGM, Mestres Gonzalvo C, de Wit HAJM, van der Kuy PHM, Janknegt R, Verhey F, Schols JMGA, Stehouwer CDA, Mulder WJ. A Survey on Medication reviews in elderly patients: substantial variation in dialy practice. J Gerontol Geriat Res. 2013;2(4):133.

13. Mestres Gonzalvo C, Hurkens KPGM, de Wit HAJM, Janknegt R, Schols JMGA, Mulder WJ, Verhey FR, Winkens B, van der Kuy PHM. Covariates that influence the quality of a medication review. Eur J Hosp Pharm. 2013;0:1-4.

14. van Oijen BPC, Janknegt R, Wit HAJM, Peters F, Schouten H, van der Kuy PHM. Medication surveillance on intravenous cytotoxic agents: a retrospective study. Int J Clin Pharm. 2013;35(4):554-9.

15. Hurkens KPGM, de Wit HAJM, Mestres Gonzalvo C, van der Kuy PHM, Janknegt R, Verhey FR, Schols JMGA, Mulder WJ. Medicatiemanagement bij de oudere patiënt: de ontwikkeling van SCREEN. Tijdschrift voor Ouderengeneeskunde.2012;3:121-5. 
Curriculum vitae 

Hugo Adrianus Johannes Maria de Wit was born on July 20, 1983 in Haren, the Netherlands. In 2001 he obtained his VWO diploma at the Sint Maartenscollege in Voorburg. In the same year he started his study Pharmacy at the Utrecht University. During his pharmaceutical studies he spent several months abroad. He completed two research projects (2004 \& 2005) at the University of Warwick, UK, and worked for 6 months in Montpellier, France, at the Institut des Neurosciences (2006). He completed his Bachelor studies in 2004 and his Master studies in 2007.

After obtaining his Pharmacy degree, Hugo worked from 2007-2010 as pharmacist/ pharmacy manager in the public pharmacy 'Prelude', Alphen aan den Rijn, part of the Pharmacy group 'Sprint Apotheken'. During this time he attended and completed a traineeship for community pharmacists and was formally registred as 'community pharmacist' in 2009. In 2010 he commenced his specialisation in Hospital Pharmacy at the Orbis Medical Centre, Sittard (supervisor: PH M Van der Kuy). During this specialisation he began the research described in this thesis.

After having completed the specialisation in hospital pharmacy, Hugo started to work at the Atrium Medical Centre, Parkstad. His main interests are clinical rules, antibiotica stewardship, and fellow practitioner. Since February 2014 Hugo combines his daily work with a clinical pharmacology program at the Zuyderland Medical Centre.

Hugo's hobbies include surfing, swimming, and bicycling. He hopes to complete an Ironman 70.3 next year. 
Hugo Adrianus Johannes Maria de Wit werd geboren op 20 juli 1983 te Haren, Nederland. In 2001 behaalde hij zijn VWO-diploma aan het Sint Maartenscollege in Voorburg, waarna hij begon aan zijn studie Farmacie aan de Universiteit van Utrecht. Tijdens zijn studie bracht hij enkele maanden in het buitenland door. Hij voltooide twee onderzoeksprojecten (2004 \& 2005) aan de Universiteit van Warwick, Groot-Brittannië, en werkte gedurende 6 maanden in Montpellier, Frankrijk, aan het Institut des Neurosciences (2006). Hij behaalde zijn bachelor Farmacie in 2004 en zijn Master in 2007.

$\mathrm{Na}$ het voltooien van zijn studie werkte Hugo van 2007-2010 als beherend apotheker in de openbare apotheek 'Prelude', Alphen aan den Rijn, onderdeel van de Apotheek groep 'Sprint Apotheken'. Gedurende deze tijd volgde hij een traineeship tot openbaar apotheker en de registratie volgde in 2009.

In 2010 begon hij zijn specialisatie tot ziekenhuisapotheker in het Orbis Medisch Centrum (huidige naam: Zuyderland Medisch Centrum) te Sittard (opleider: PH M Van der Kuy). Tijdens deze specialisatie begon hij ook aan het onderzoek zoals beschreven in dit proefschrift.

$\mathrm{Na}$ het afronden van zijn specialisatie startte Hugo in het Atrium Medisch Centrum Parkstad (huidige naam: Zuyderland Medisch Centrum) als ziekenhuisapotheker. Zijn belangrijkste interessegebieden zijn klinische beslisregels, antibiotica stewardship en medebehandelaarschap. Sinds februari 2014 combineert Hugo zijn werk met een opleiding tot klinisch farmacoloog in het Zuyderland Medisch Centrum.

Hugo's hobby's zijn surfen, zwemmen en fietsen. Zijn doel is om volgend jaar een Ironman 70.3 te voltooien. 


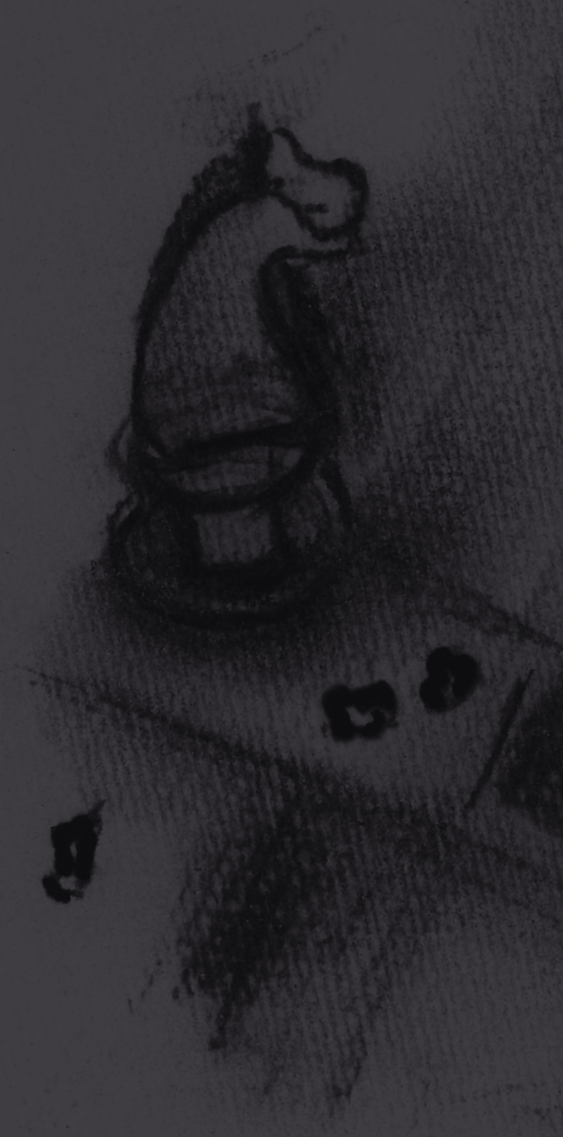

